TRANSACTIONS OF THE

AMERICAN MATHEMATICAL SOCIETY

Volume 364, Number 2, February 2012, Pages 827-866

S 0002-9947(2011)05331-6

Article electronically published on September 1,2011

\title{
ON THE $n$-BACK-AND-FORTH TYPES OF BOOLEAN ALGEBRAS
}

\author{
KENNETH HARRIS AND ANTONIO MONTALBÁN
}

\begin{abstract}
The objective of this paper is to uncover the structure of the back-and-forth equivalence classes at the finite levels for the class of Boolean algebras. As an application, we obtain bounds on the computational complexity of determining the back-and-forth equivalence classes of a Boolean algebra for finite levels. This result has implications for characterizing the relatively intrinsically $\Sigma_{n}^{0}$ relations of Boolean algebras as existential formulas over a finite set of relations.
\end{abstract}

\section{INTRODUCTION}

The program of effective mathematics is concerned with computational aspects of mathematical structures. One asks questions such as the following: what is the simplest way to represent a certain structure? Given two isomorphic computable structures, what conditions guarantee that some isomorphism is computable? Or, that every isomorphism is computable? What conditions on a relation in a computable structure guarantee that the image of the relation in any computable copy of the structure is computable? Or, that the image is computably enumerable? How do solutions to these problems generalize within the hyperarithmetic hierarchy?

Each of the questions in the previous paragraph has been resolved by providing an effective version of the classical construction of isomorphisms by the back-andforth method. (See AK00, Chapters 16-18] for a statement and resolution of each of these questions.) Answering these questions for a specific class of structures requires an analysis of the complexity of the back-and-forth relations on those structures. This paper provides such an analysis of the back-and-forth relations at finite levels for Boolean algebras in order to apply the effective back-and-forth methods for building computable copies of Boolean algebras.

The back-and-forth method, or the method of extension of partial isomorphisms, originated with Cantor's stepwise construction of an isomorphism between any two countable dense linear orders without endpoints. The method is basic in model theory, for example, in the proof that any two countably homogeneous models realizing the same types are isomorphic. Langford extended the application of the method to show that any two dense linear orders without endpoints were elementarily equivalent (regardless of their cardinality). Ehrenfeucht and Fraïssé gave a

Received by the editors October 2, 2007 and, in revised form, January 28, 2008, August 13, 2008, June 9, 2009, June 29, 2009, February 18, 2010 and February 19, 2010.

2010 Mathematics Subject Classification. Primary 03D80; Secondary 03C57.

Key words and phrases. Boolean algebra, back-and-forth, invariant.

The second author was partially supported by NSF Grants DMS-0600824 and DMS-0901169 and by the Marsden Foundation of New Zealand, via a postdoctoral fellowship.

(c) 2011 American Mathematical Society 827

Reverts to public domain 28 years from publication 
purely algebraic characterization of elementary equivalence in first-order structures in terms of families of partial isomorphisms with a (one-at-a-time) back-and-forth property. Karp generalized this characterization and showed that the mathematical framework where the technique is naturally expressed is the infinitary logic $L_{\infty \omega}$. For more details on this history and Karp's contribution, see Bar73, or Dic85, Section 4].

The investigation of general back-and-forth techniques in the context of effective mathematics started with the work of Ash on $\alpha$-systems in Ash86b and Ash86a. The objects studied are countable structures over a computable language, and the constructions are over computable ordinals. Ash and Knight produced a general metatheorem for applying the $\alpha$-system machinery. The backbone for applying the metatheorem is a hierarchy of back-and-forth relations on finite approximations of the construction. The construction in the Ash and Knight metatheorem can be viewed as a hierarchy of worker constructions, where the worker at each level has limited information about the structure to be constructed; the back-and-forth relations ensure that mistakes made by workers at lower levels can be repaired at higher levels later in the construction. Success in the construction depends on the workers at each level having access to the appropriate back-and-forth relations to guide their constructions. (See AK00, Chapters 13 and 14] for more details.) To apply the metatheorem to a particular type of structure, one has to understand how the back-and-forth relations behave for that structure. These relations have been determined for some types of structures. Ash studied the back-and-forth relations on well-orderings [Ash87] and on superatomic Boolean algebras Ash86b; Ash and Knight AK00 studied vector spaces; Barker Bar95 and Calvert Cal05 studied reduced Abelian p-groups; and Csima, Montalbán and Shore CMS06 analyzed saturated Boolean algebras.

We are interested in the back-and-forth relations for Boolean algebras at finite levels of the hierarchy of back-and-forth relations. Let $L$ be the language of Boolean algebras and $L_{\omega_{1} \omega}$ be the infinitary language allowing countable conjunctions and disjunctions, but a finite nesting of quantifiers. The subclasses of infinitary formulas $\Sigma_{n}$ and $\Pi_{n}$ have $n$ alternations of existential and universal quantifiers (counting infinite disjunctions as existential quantification and infinite conjunctions as universal quantification). We write $\Sigma_{n}(\mathcal{A})\left(\Pi_{n}(\mathcal{A})\right)$ for the set of $\Sigma_{n}\left(\Pi_{n}\right)$ sentences true in $\mathcal{A}$. The $n$-back-and-forth relation between two Boolean algebras holds when $\Pi_{n}(\mathcal{A}) \subseteq \Pi_{n}(\mathcal{B})$ (or, equivalently, when $\Sigma_{n}(\mathcal{B}) \subseteq \Sigma_{n}(\mathcal{A})$; see Subsection 2.3). We write $\mathcal{A} \leq_{n} \mathcal{B}$ when either of these two conditions is obtained and $\mathcal{A} \equiv_{n} \mathcal{B}$ when both $\mathcal{A} \leq_{n} \mathcal{B}$ and $\mathcal{B} \leq_{n} \mathcal{A}$.

Our first aim in this paper is to provide a combinatorial characterization of the $n$-back-and-forth equivalence classes. We aim for a map $T_{n}(\cdot)$ on Boolean algebras so that

$$
\mathcal{A} \equiv_{n} \mathcal{B} \Longleftrightarrow T_{n}(\mathcal{A})=T_{n}(\mathcal{B})
$$

Furthermore, we want the object given by $T_{n}(\mathcal{A})$ to be computationally simpler than $\Sigma_{n}(\mathcal{A})$, so that determining whether $T_{n}(\mathcal{A})=T_{n}(\mathcal{B})$ is, ideally, computable. One of the main results of this paper is to produce invariants which satisfy these two conditions (Section 7). The definition of these invariants is based on a modification of a topological invariant defined by Flum and Ziegler FZ80. To each countable Boolean algebra $\mathcal{A}$ we assign an object, $T_{n}(\mathcal{A})$, which encodes how $\mathcal{A}$ may be partitioned into a finite join of elements. Let $\mathbf{I N V}_{n}$ be the set of possible values 
of $T_{n}(\mathcal{A})$ (we will show that $\mathbf{I N V _ { n }}$ is countable). On the elements of $\mathbf{I N V _ { n }}$, we define a partial ordering $\leq_{n}$, a binary operation + , and a function $(\cdot)_{n-1}: \mathbf{I N V}_{n} \rightarrow$ $\mathbf{I N V}_{n-1}$ satisfying the following properties for all Boolean algebras $\mathcal{A}$ and $\mathcal{B}$ :

$$
\begin{aligned}
& T_{n}(\mathcal{A}) \leq_{n} T_{n}(\mathcal{B}) \Longleftrightarrow \mathcal{A} \leq_{n} \mathcal{B}, \\
& T_{n}(\mathcal{A})+T_{n}(\mathcal{B})=T_{n}(\mathcal{A} \oplus \mathcal{B}), \\
& \left(T_{n}(\mathcal{A})\right)_{n-1}=T_{n-1}(\mathcal{A})
\end{aligned}
$$

(where $\mathcal{A} \oplus \mathcal{B}$ is the directed sum of $\mathcal{A}$ and $\mathcal{B}$ ). The definitions are purely combinatorial, and the structures (given for each $n$ )

$$
\left(\mathbf{I N V}_{n}, \leq_{n},+,(\cdot)_{n}\right)
$$

are uniformly computable in $n$.

The key to our investigation of the $n$-back-and-forth types are $n$-indecomposable Boolean algebras:

Definition 3.1. A Boolean algebra $\mathcal{A}$ is $n$-indecomposable if for any partition of $\mathcal{A}$ into subalgebras, $\mathcal{A}=\mathcal{A}_{0} \oplus \ldots \oplus \mathcal{A}_{k}$, there is an $i \leq k$ such that $\mathcal{A} \equiv_{n} \mathcal{A}_{i}$.

We summarize the main results about $n$-indecomposable algebras.

(a) For each $n$, there are only finitely many $n$-back-and-forth equivalence classes among the $n$-indecomposable algebras (Theorem 3.17).

(b) Every Boolean algebra can be decomposed into a finite sum of $n$-indecomposable subalgebras (Theorem 3.13).

(c) These decompositions fully determine the $n$-back-and-forth equivalence classes among all Boolean algebras (Lemma 3.14).

$\mathbf{B F}_{n}$ will denote the set of invariants in $\mathbf{I N V}_{n}$ which correspond to $n$-indecomposable Boolean algebras. By (b) and (c), $\mathbf{I N V} \mathbf{V}_{n}$ is finitely generated by $\mathbf{B} \mathbf{F}_{n}$ under + . The general $n$-back-and-forth invariants, $\mathbf{I N V}_{n}$, record all the possible ways an algebra can be partitioned into finitely many $n$-indecomposable subalgebras, up to $n$-back-and-forth equivalence.

A second aim of this paper is to provide an analysis of the computational complexity of the relations on a Boolean algebra $\mathcal{A}$ determined by $T_{n}\left(\mathcal{A}\lceil a) \geq_{n} \sigma\right.$ for $a \in \mathcal{A}$ and $\sigma \in \mathbf{I N V}_{n}$. We use, for this purpose, the hierarchy of computable infinitary formulas, $\Sigma_{n}^{c}$ and $\Pi_{n}^{c}$, which restricts conjunctions and disjunctions to computably enumerable sets of formulas (see Subsection 2.2). The important property of this class is that for any formula $\phi(x) \in \Pi_{n}^{c}$, the relation $\{a \in \mathcal{A}: \mathcal{A} \models \phi(a)\}$ is $\Pi_{n}^{0}$. We show that for each $\sigma \in \mathbf{I N V}_{n}$, there is a $\Pi_{n}^{c}$ formula $\phi_{\sigma}^{c}(x)$ and a $\Pi_{n+1}^{c}$ formula $\psi_{\sigma}^{c}(x)$ such that for each $a \in \mathcal{A}$,

$$
\begin{aligned}
\mathcal{A}=\phi_{\sigma}^{c}(a) & \Longleftrightarrow T_{n}\left(\mathcal{A}\lceil a) \geq_{n} \sigma,\right. \\
\mathcal{A}=\psi_{\sigma}^{c}(a) & \Longleftrightarrow T_{n}\left(\mathcal{A}\lceil a) \leq_{n} \sigma\right.
\end{aligned}
$$

(Lemmas 8.10 and 8.11 ).

One application of these invariants is a quantifier elimination result for $\Sigma_{n+1}^{c}$ formulas. Let $\mathrm{R}_{\sigma}$ be the unary predicate which holds for an element $a \in \mathcal{A}$ if and only if $T_{n}\left(\mathcal{A}\lceil a) \geq_{n} \sigma\right.$. Then, from the previous paragraph, the set $\{a \in \mathcal{A}: \mathcal{A} \models$ $\left.\mathrm{R}_{\sigma}(a)\right\}$ is a $\Pi_{n}^{0}(\mathcal{A})$ subset of $\mathcal{A}$ for any $\sigma \in \mathbf{I N V}_{n}$. We show that every $\Sigma_{n+1}^{c}$ formula in the language of Boolean algebras is equivalent to a $\Sigma_{1}^{0}$ formula over the finitely many additional predicates $\mathrm{R}_{\alpha}$, for $\alpha \in \mathbf{B F}_{n}$. 
Theorem 8.12, Let $\mathcal{B}$ be a Boolean algebra, $R \subseteq \mathcal{B}$ and $n \in \omega$. The following are equivalent:

(1) $R$ is relatively intrinsically $\Sigma_{n+1}$. That is, if $\mathcal{A} \cong \mathcal{B}$ and $(\mathcal{A}, Q) \cong(\mathcal{B}, R)$, then $Q$ is a $\Sigma_{n+1}^{0}(\mathcal{A})$ subset of $\mathcal{A}$.

(2) $R$ is explicitly $\Sigma_{n+1}$. That is, $R$ can be defined in $\mathcal{B}$ by a computable infinitary $\Sigma_{n+1}^{c}$ formula.

(3) There is a $0^{(n)}$-computable sequence $\left\{\varphi_{i}: i \in \omega\right\}$ of finitary $\Sigma_{1}$ formulas over the predicates $\mathrm{R}_{\alpha}$, for $\alpha \in \mathbf{B F}_{n}$, such that

$$
x \in R \Longleftrightarrow \bigvee_{i \in \omega} \varphi_{i}(x) \text {. }
$$

The equivalence between the first two statements is due to Ash, Knight, Manasse, Slaman, and Chisholm (see AK00, Theorem 10.1]).

This theorem says that the finitely many predicates, $\mathbf{R}_{\alpha}$ for $\alpha \in \mathbf{B F}_{n}$, essentially give all the structural information about a Boolean algebra that can be computed in $n$ Turing jumps. The bound on the complexity of the sequence $\left\{\varphi_{i}: i \in \omega\right\}$ cannot be improved from $0^{(n)}$ because the $\Sigma_{n+1}^{c}$ formulas are strong enough to code any $\Sigma_{n+1}^{0}$ set (see Section 2.2 for further details).

We also have a characterization of when the $\Sigma_{n+1}^{c}$-diagram of a Boolean algebra $\mathcal{A}$ (that is, the $\Sigma_{n+1}^{c}$ sentences with constants from $\mathcal{A}$ which are true in $\mathcal{A}$ ) is $\Sigma_{n+1}^{0}$.

Theorem 8.13. Let $\mathcal{A}$ be a presentation of a Boolean algebra. The following are equivalent:

(a) The $\Sigma_{n+1}^{c}$-diagram of $\mathcal{A}$ is a $\Sigma_{n+1}^{0}$ set of formulas.

(b) The relations $\mathrm{R}_{\alpha}(\mathcal{A})$ are computable in $0^{(n)}$ for each $\alpha \in \mathbf{B F}_{n}$.

A Boolean algebra is n-approximable if it satisfies either of the conditions in the theorem. Note that being n-approximable is a property of a presentation of a Boolean algebra rather than a property of the isomorphism type of a Boolean algebra. Of course, every computable Boolean algebra is $n$-approximable, and every $\operatorname{low}_{n}$ Boolean algebra is also $n$-approximable. The main open question is whether every $n$-approximable Boolean algebra has a computable copy. This is true for $n \in\{1,2,3,4\}$ : Downey and Jockusch DJ94 proved that every low Boolean algebra has a computable copy; this was extended by Thurber Thu95 to low 2 Boolean algebras and by Knight and Stob [KS00 to low 4 . Each proof proceeds by showing that any $n$-approximable Boolean algebra has an $(n-1)$-approximable copy, for $n \in\{1,2,3,4\}$. It is still open whether every 5 -approximable Boolean algebra has a computable copy. Here it is sufficient to show that every 5 -approximable Boolean algebra has a 4-approximable copy. A motivation for this work is to obtain a better understanding of the presentations of $n$-approximable algebras in order to provide a means of determining whether every $\operatorname{low}_{n}$ Boolean algebra has a computable copy. To this end, we have enumerated $\mathbf{B F}_{n}$ for $n \in\{1,2,3,4,5\}$ in Section 6 .

We mention two antecedents of our work. Jockusch and Soare JS94 produced finitely many invariants for the back-and-forth relations on Boolean algebras through level two, as well as topological invariants for the Stone space of a Boolean algebra along the same lines as Flum and Ziegler [FZ80. Pavel Alaev Ala04. described the back-and-forth relations on Boolean algebras through level four by means of numerical invariants and used these invariants in combination with an extension of the Ash-Knight $\alpha$-system machinery. 


\section{Preliminaries}

2.1. Boolean algebras. Boolean algebras will be taken to be countable and in the signature $\wedge, \vee,-, 0,1$, but otherwise we follow the standard reference Mon89. Boolean algebras will be denoted by $\mathcal{A}, \mathcal{B}, \mathcal{C}$ and their elements by $a, b, c$. We abbreviate $a \leq b$ for $a \wedge b=a$ and denote the relative algebra by $\mathcal{A}\lceil a=\{b \in \mathcal{A}: b \leq a\}$.

A partition of an element $a$ in a Boolean algebra $\mathcal{A}$ is a finite sequence $a_{0}, \ldots, a_{k}$ of pairwise disjoint elements (that is, $a_{i} \wedge a_{j}=0$ when $i \neq j$ ) such that $a=$ $a_{0} \vee \ldots \vee a_{k}$; a partition of a Boolean algebra $\mathcal{A}$ is a partition of its unit, $1_{\mathcal{A}}$. We write $a=\left(a_{i}\right)_{i \leq k}$ to mean that $a_{0}, \ldots, a_{k}$ is a partition of $a$. If $\left(a_{i}\right)_{i \leq k}$ is any sequence of elements from $\mathcal{A}$, then a sequence $\left(b_{i, j}\right)_{i \leq k, j \leq \ell_{i}}$ is a refinement of $\left(a_{i}\right)_{i \leq k}$ if $a_{i}=\bigvee_{j \leq \ell_{i}} b_{i, j}$. If $\left(a_{i}\right)_{i \leq k}$ and $\left(b_{j}\right)_{j \leq \ell}$ are partitions, then there is a common refinement partition $\left(c_{i, j}\right)_{i \leq k, j \leq \ell}$ (where $c_{i, j}=a_{i} \wedge b_{j}$ ) satisfying $a_{i}=\bigvee_{j \leq \ell} c_{i, j}$ and $b_{j}=\bigvee_{i \leq k} c_{i, j}$. Note that any finite sequence of elements $\left(a_{i}\right)_{i \leq k}$ can be refined to a partition $\left(c_{j}\right)_{j \leq 2^{k}}$, where each $c_{j}$ is of the form $\bigwedge_{i \leq k} b_{i}$ and each $b_{i}$ is $a_{i}$ or $-a_{i}$.

An ultrafilter $U$ of a Boolean algebra $\mathcal{A}$ is a nonempty, proper subset of $\mathcal{A}$ satisfying

(Uf.a) For any partition $\left(a_{i}\right)_{i \leq k}$ of $\mathcal{A}, a_{i} \in U$ for some $i \leq k$.

(Uf.b) For all $a \in U$, if $b \geq a$, then $b \in U$.

(Uf.c) For all $a, b \in U, a \wedge b \in U$.

For any $a \in U$, we write $U\lceil a$ for the set $\{b \in U: b \leq a\}$. If $U$ is an ultrafilter on $\mathcal{A}$, then $U \uparrow a$ is an ultrafilter on $\mathcal{A} \uparrow a$. Conversely, if $U$ is an ultrafilter on $\mathcal{A}\lceil a$, then there is a unique ultrafilter $\bar{U}$ on $\mathcal{A}$ with $U \subseteq \bar{U}$; it is given by $\bar{U}=\{x \in \mathcal{A}: x \wedge a \in U\}$. The set of ultrafilters of $\mathcal{A}$ is denoted by $\operatorname{Ult}(\mathcal{A})$. There is a natural topology on $\operatorname{Ult}(\mathcal{A})$, the Stone space of $\mathcal{A}$, determined by the basic open sets $\mathcal{O}_{a}=\{U \in \operatorname{Ult}(\mathcal{A}): a \in U\}$ for $a \in \mathcal{A}$. This topological space is Hausdorff, compact and zero-dimensional; see [Mon89, §7].

2.2. Syntax. We will be working in a predicate language appropriate for countable Boolean algebras, $L_{\omega_{1} \omega}$, which allows conjunctions and disjunctions over arbitrary countable sets of formulas and finite nesting of quantifiers. The nonlogical signature includes the Boolean operators $\wedge, \vee,-$, and constants 0,1 ; the logical symbols are the connectives $\wedge$ (countable conjunction), $\bigvee$ (countable disjunction), $\neg$ (negation), the quantifiers $\exists$ and $\forall$, and $=$ (identity). We will also write $a=a_{0} \dot{V} \ldots \dot{V} a_{k}$ to emphasize that $a_{0}, \ldots, a_{k}$ is a partition of $a$. All formulas have only finitely many distinct free variables. Satisfaction of formulas of $L_{\omega_{1} \omega}$ in a Boolean algebra is defined in the natural way, and $\mathcal{A}=\phi\left(a_{0}, \ldots, a_{k}\right)$ means $\mathcal{A}$ satisfies the formula $\phi\left(x_{0}, \ldots, x_{k}\right)$ at the tuple of elements $\left(a_{0}, \ldots, a_{k}\right)$.

The subclass of formulas in normal form will be denoted by $\Sigma_{n}$ and $\Pi_{n}$ and defined inductively for $n \in \omega$. The $\Sigma_{0}$ and $\Pi_{0}$ formulas are the finitary open formulas (that is, quantifier-free formulas with no infinite disjunctions or conjunctions). For $n>0$, a $\Sigma_{n}$ formula is a countable disjunction of formulas of the form $\exists \bar{x} \psi$, where $\bar{x}$ is a finite sequence of variables and $\psi \in \Pi_{m}$ for some $m<n$. Similarly, a $\Pi_{n}$ formula is a countable conjunction of formulas of the form $\forall \bar{x} \psi$, where $\bar{x}$ is a finite sequence of variables and $\psi \in \Sigma_{m}$ for some $m<n$. The negation of a $\Sigma_{n}$ formula is logically equivalent to a $\Pi_{n}$ formula (see [AK00, Lemma 6.1]). 
The class of computable infinitary formulas, $L_{\omega_{1} \omega}^{c}$, was introduced in Ash86b and restricts conjunctions and disjunctions to computably enumerable sets of formulas. We provide an informal description of the class $\Sigma_{n}^{c}$ and $\Pi_{n}^{c}$ for $n \in \omega$, although the definitions can be extended over the computable ordinals. The $\Sigma_{0}^{c}$ and $\Pi_{0}^{c}$ formulas are the finitary open formulas. The computable infinitary $\Sigma_{n+1}^{c}$ formulas are restricted to disjunctions over a c.e. set of formulas of the form $\exists \bar{x} \psi$, where $\bar{x}$ is a finite sequence of variables and $\psi \in \Pi_{m}^{c}$ for some $m \leq n$. The computable infinitary $\Pi_{n+1}^{c}$ formulas are restricted to conjunctions over a c.e. set of formulas of the form $\forall \bar{x} \psi$, where $\bar{x}$ is a finite sequence of variables and $\psi \in \Sigma_{m}^{c}$ for some $m \leq n$. The important fact about computable infinitary formulas is that the complexity of the formula matches the complexity of its interpretation. If $\varphi\left(x_{0}, \ldots, x_{k}\right) \in \Sigma_{n}^{c}$, then the set of tuples $\left(a_{0}, \ldots, a_{k}\right)$ for which $\mathcal{A} \models \varphi\left(a_{0}, \ldots, a_{k}\right)$ is $\Sigma_{n}^{0}(\mathcal{A})$, and if $\varphi\left(x_{0}, \ldots, x_{k}\right) \in \Pi_{n}^{c}$, then the set of tuples $\left(a_{0}, \ldots, a_{k}\right)$ for which $\mathcal{A} \equiv \varphi\left(a_{0}, \ldots, a_{k}\right)$ is $\Pi_{n}^{0}(\mathcal{A})$. See [AK00, Chapter 7] for a formal definition of the computable infinitary formulas and their properties. Our use of the notation $\Sigma_{n}^{c}$ and $\Pi_{n}^{c}$ was used in AK90, although not in AK00.

The sentences in $\Sigma_{n+1}^{c}$ are strong enough to code any $\Sigma_{n+1}^{0}$ set in the following sense. For any $\Sigma_{n+1}^{0}$ set $X=W_{e}^{0^{(n)}}$ there is a computable sequence of $\Sigma_{n+1}^{c}$ sentences $\left\langle\theta_{e, k}\right\rangle_{k \in \omega}$ such that $k \in X$ implies $\theta_{k} \equiv \top$ and $k \notin X$ implies $\theta_{k} \equiv \perp$ (see [AK00, Theorem 7.9]). Let $\psi_{k}$ be a $\Sigma_{2}^{c}$ sentence stating there are exactly $k$ elements in the universe; that is,

$$
\exists x_{1} \ldots x_{k} \forall y\left[\bigvee_{1 \leq i \leq k} y=x_{i} \wedge \bigwedge_{1 \leq i<j \leq k} x_{i} \neq x_{j}\right]
$$

For $n \geq 1$, the sentence $\theta_{e, k} \wedge \psi_{k}$ is $\Sigma_{n+1}^{c}$. By dovetailing computable disjunctions, the sentence $\varphi_{e}:=\bigvee_{k \in \omega}\left(\theta_{e, k} \wedge \psi_{k}\right)$ can be rewritten equivalently as a $\Sigma_{n+1}^{c}$ sentence. Now, $\mathcal{A} \models \varphi_{e}$ if and only if $|\mathcal{A}|=k$ for some $k \in X$.

2.3. Back-and-forth relations. We refer the reader to AK00, Chapter 15, especially $\$ 15.3 .4]$ for more details on back-and-forth relations in Boolean algebras.

The back-and-forth relation on Boolean algebras, $\mathcal{A} \leq_{n} \mathcal{B}$, was defined in the introduction as $\Pi_{n}(\mathcal{A}) \subseteq \Pi_{n}(\mathcal{B})$, or equivalently $\Sigma_{n}(\mathcal{B}) \subseteq \Sigma_{n}(\mathcal{A})$. However, it is more convenient to use an alternative characterization. The two accounts of $\leq_{n}$ converge in Theorem 2.3 ,

Definition 2.1. The relation $\leq_{n}$ on Boolean algebras is defined by recursion on $n \in \omega$. Let $\mathcal{A}$ and $\mathcal{B}$ be Boolean algebras. Define $\mathcal{A} \leq_{0} \mathcal{B}$ if either both $\mathcal{A}$ and $\mathcal{B}$ are the one-element Boolean algebra or neither is. Also, define $\mathcal{A} \leq_{n+1} \mathcal{B}$ if for every partition $\left(b_{i}\right)_{i \leq k}$ of $\mathcal{B}$, there is a partition $\left(a_{i}\right)_{i \leq k}$ of $\mathcal{A}$ such that $\mathcal{B} \uparrow b_{i} \leq_{n} \mathcal{A} \uparrow a_{i}$ for all $i \leq k$. We write $\mathcal{A} \equiv_{n} \mathcal{B}$ when both $\mathcal{A} \leq_{n} \mathcal{B}$ and $\mathcal{B} \leq_{n} \mathcal{A}$.

For $a \in \mathcal{A}$ and $b \in \mathcal{B}$ define $a \leq_{n} b$ if $\mathcal{A} \uparrow a \leq_{n} \mathcal{B} \uparrow b$. For any partition $\left(a_{i}\right)_{i \leq k}$ of $\mathcal{A}$ and $\left(b_{i}\right)_{i \leq k}$ of $\mathcal{B}$, we write $\left(a_{i}\right)_{i \leq k} \leq_{n}\left(b_{i}\right)_{i \leq k}$ if $a_{i} \leq_{n} b_{i}$ for each $i \leq k$. Therefore, $\mathcal{A} \leq_{n+1} \mathcal{B}$ if and only if for every partition $\left(b_{i}\right)_{i \leq k}$ of $\mathcal{B}$, there is a partition $\left(a_{i}\right)_{i \leq k}$ of $\mathcal{A}$ with $\left(b_{i}\right)_{i \leq k} \leq_{n}\left(a_{i}\right)_{i \leq k}$.

The following is essentially from [AK00, Lemma 15.12].

Lemma 2.2. Let $\left(a_{i}\right)_{i \leq k}$ be a partition of $\mathcal{A}$ and $\left(b_{i}\right)_{i \leq k}$ be a partition of $\mathcal{B}$ satisfying $(a)_{i \leq k} \leq_{n}(b)_{i \leq k}$. Then $\mathcal{A} \leq_{n} \mathcal{B}$. 
Proof. The proof is by induction on $n \in \omega$. The result is trivial for the basis case $n=0$. Suppose the statement of the lemma is true for $n$. Let $\left(a_{i}\right)_{i \leq k}$ be a partition of $\mathcal{A}$ and $\left(b_{i}\right)_{i \leq k}$ be a partition of $\mathcal{B}$ satisfying $\left(a_{i}\right)_{i \leq k} \leq_{n+1}\left(b_{i}\right)_{i \leq k}$. To show $\mathcal{A} \leq_{n+1} \mathcal{B}$, consider any partition $\left(d_{j}\right)_{j \leq \ell}$ of $\mathcal{B}$, and let $\left(b_{i, j}\right)_{i \leq k, j \leq \ell}$ be the common refinement with $\left(b_{i}\right)_{i \leq k}$ so that $d_{j}=\bigvee_{i \leq k} b_{i, j}$ and $b_{i}=\bigvee_{j \leq \ell} b_{i, j}$. Then, for each $i \leq k$ there is a partition $\left(a_{i, j}\right)_{j \leq \ell}$ of $a_{i}$ where $a_{i, j} \geq_{n} b_{i, j}$. Let $c_{j}=\bigvee_{i \leq k} a_{i, j}$ so that by the inductive hypothesis $c_{j} \geq_{n} d_{j}$. Thus, $\left(c_{j}\right)_{j \leq \ell}$ is a partition of $\mathcal{A}$ with $\left(c_{j}\right)_{j \leq \ell} \geq_{n}\left(d_{j}\right)_{j \leq \ell}$. Since $\left(d_{j}\right)_{j \leq \ell}$ was arbitrary, we have $\mathcal{A} \leq_{n+1} \mathcal{B}$ by Definition 2.1 .

The next theorem connects the syntactic characterization of back-and-forth relations from the introduction with the characterization by partitions (see AK00, Proposition 15.1]).

Theorem 2.3. Let $\left(a_{i}\right)_{i \leq k}$ be a partition of $\mathcal{A}$ and $\left(b_{i}\right)_{i \leq k}$ be a partition of $\mathcal{B}$. Then for any $n \in \omega$ the following are equivalent:

(1) $\left(a_{i}\right)_{i \leq k} \leq_{n}\left(b_{i}\right)_{i \leq k}$.

(2) The $\Sigma_{n}$ formulas true of $\left(b_{i}\right)_{i \leq k}$ in $\mathcal{B}$ are true of $\left(a_{i}\right)_{i \leq k}$ in $\mathcal{A}$.

(3) The $\Pi_{n}$ formulas true of $\left(a_{i}\right)_{i \leq k}$ in $\mathcal{A}$ are true of $\left(b_{i}\right)_{i \leq k}$ in $\mathcal{B}$.

Since $\Sigma_{n}, \Pi_{n} \subset \Sigma_{n+1}$, we have the following.

Corollary 2.4. If $\mathcal{A} \leq_{n+1} \mathcal{B}$, then $\mathcal{A} \equiv_{n} \mathcal{B}$.

\section{INDECOMPOSABLE ALGEBRAS}

Indecomposable Boolean algebras are the essence of our analysis of back-andforth invariants. Heuristically, an indecomposable algebra is one which is similar to one of its subalgebras regardless of how it is split, where similarity captures some equivalence relation on Boolean algebras of interest. For example, when the relation is that of an isomorphism, a pseudo-indecomposable Boolean algebra $\mathcal{A}$ is one satisfying $\mathcal{A} \cong \mathcal{A}\lceil a$ or $\mathcal{A} \cong \mathcal{A} \uparrow-a$ for each $a \in \mathcal{A}$. In this paper we are interested in the family of back-and-forth equivalence relations $\equiv_{n}$ for $n \in \omega$.

Definition 3.1. A Boolean algebra $\mathcal{A}$ is $n$-indecomposable if for any partition $\left(a_{i}\right)_{i \leq k}$ of $\mathcal{A}$, there is an $i$ with $\mathcal{A} \equiv_{n} \mathcal{A}\left\lceil a_{i}\right.$.

We will show in Theorem 4.1 that this definition is equivalent to the condition that $\mathcal{A} \equiv_{n} \mathcal{A}\left\lceil a\right.$ or $\mathcal{A} \equiv_{n} \mathcal{A}\lceil-a$ for any $a \in \mathcal{A}$. We will also say more at the end of Section 7 about the relation between pseudo-indecomposable algebras and the notion of indecomposable captured in this definition.

It is more convenient to approach the characterization of $n$-indecomposable algebras from a different viewpoint. We start with the topological characterization of the $n$-types of accumulation points in the Stone space of the algebra and impose an additional relation, $\leq_{n}$, on these $n$-types. It follows from the work of Flum and Ziegler FZ80, that for every ultrafilter $U$ of a Boolean algebra $\mathcal{A}$, there is an $a \in U$ (the basic neighborhood $\mathcal{O}_{a}$ of $U$ in the Stone space) such that for every $b \in U$ with $b \leq a$ (equivalently in the Stone space, $\mathcal{O}_{b} \subseteq \mathcal{O}_{a}$ ), the Boolean algebras $\mathcal{A}\lceil b$ are all $\equiv_{n}$-equivalent. (See [Hei81] or [FZ80, Part II, $\left.\S 1, \mathrm{C}\right]$ for further discussion of the classification of the accumulation points in a Stone space. Knowledge of this material is not presupposed in what follows.) We classify accumulation points in the Stone space using equivalence classes under $\equiv_{n}$, the $n$-back-and-forth types. 
We use these types to pick out the $n$-indecomposable algebras (see Definition 3.5). The key theorems are that every Boolean algebra can be decomposed into finitely many $n$-indecomposable types (Theorem 3.13) and that the $n$-back-and-forth types are invariants for $n$-indecomposable algebras (Theorem 3.17). These two theorems allow us to describe invariants for the $n$-back-and-forth equivalence classes of all Boolean algebras by decomposing algebras into finitely many $n$-indecomposable subalgebras (see Section 77). The heuristic approach to $n$-indecomposability from Definition 3.1 and our more technically involved definition in terms of accumulation points (Definition 3.5) converge in Theorem 4.1. In Section 6] we provide a description of all $n$-back-and-forth types for $n \leq 5$.

3.1. Indecomposable types. In Definition 3.6, we will define finite partial orderings $\left(\mathbf{A} \mathbf{B} \mathbf{F}_{n}, \leq_{n}\right)$, where the elements of $\mathbf{A} \mathbf{B} \mathbf{F}_{n+1}$ are subsets of $\mathbf{A} \mathbf{B} \mathbf{F}_{n}$. The reader could jump ahead and read Definition 3.6 now, although it might look too technical at first. We call the elements of $\mathbf{A B F}_{n} n$-bf-types. Before actually defining these partial orderings, we define invariant maps $t_{n}: \operatorname{Ult}(\mathcal{A}) \rightarrow \mathbf{A B F}_{n}$ and commence to assign $n$-bf-types to Boolean algebras.

Definition 3.2. For $X \subset \mathbf{A B F}{ }_{n}$, we let $\max X$ be the antichain of $\leq_{n}$-maximal elements of $X$.

Definition 3.3. We assume $\mathcal{A}$ is not the trivial one element algebra. To each ultrafilter $U$ of a Boolean algebra $\mathcal{A}$ and each $n \in \omega$, we assign an $n$-bf-type as follows:

$$
\begin{aligned}
t_{0}(U) & =*, \quad \text { where } * \text { is a new symbol. } \\
\hat{t}_{n+1}(U) & =\left\{\alpha \in \mathbf{A B F}_{n}: \forall a \in U \exists V \in \operatorname{Ult} \mathcal{A}\left[V \neq U \& a \in V \& t_{n}(V)=\alpha\right]\right\} . \\
t_{n+1}(U) & =\max \hat{t}_{n+1}(U) .
\end{aligned}
$$

The definition is usefully rephrased in topological terms on the Stone space: $\hat{t}_{n+1}(U)$ consists of the elements $\alpha \in \mathbf{A} \mathbf{B F}_{n}$ such that $U$ is an accumulation point of $\{V \in$ $\left.\operatorname{Ult}(\mathcal{A}): t_{n}(V)=\alpha\right\}$. (In the Stone space, the elements $a \in U$ correspond to basic clopen neighborhoods of $U, U \in \mathcal{O}_{a}$. Therefore, $\alpha \in \hat{t}_{n+1}(U)$ if each basic clopen neighborhood of $U$ meets $\left\{V \in \operatorname{Ult}(\mathcal{A}): t_{n}(V)=\alpha\right\}$ in a point distinct from $U$.)

To each nonzero element $a \in \mathcal{A}$, we assign an $n$-bf-type as follows. For $n=0$, let $t_{0}(a)=*$. Given $t_{n}$, let $\hat{t}_{n}(a) \subset \mathbf{A} \mathbf{B} \mathbf{F}_{n}$ be the set of $\alpha \in \mathbf{A} \mathbf{B F}_{n}$ such that there are infinitely many distinct ultrafilters $V$ with $a \in V$ and $t_{n}(V)=\alpha$; then, let $t_{n+1}(a)=\max \hat{t}_{n}(a)$. (In the Stone space, $\alpha \in \hat{t}_{n+1}(a)$ if the clopen neighborhood $\mathcal{O}_{a}$ contains infinitely many points of $\left\{V \in \operatorname{Ult}(\mathcal{A}): t_{n}(V)=\alpha\right\}$.)

We extend the map to Boolean algebras $\mathcal{A}$ by $t_{n}(\mathcal{A})=t_{n}\left(1_{\mathcal{A}}\right)$.

The invariants defined in [FZ80] just iterate the function $\hat{t}_{n}$ and do not mention the antichain of maximal elements; nor do they consider any ordering on the set of invariants. The reason we need to use this refined version of their invariants is that we are seeking an invariant that corresponds to the hierarchy of $n$-back-andforth equivalence relations. Both our $n$-invariants and Flum and Ziegler's record information on how a Boolean algebra can be partitioned into $(n-1)$-invariants. Our invariants record exactly the amount of information necessary to decide the $n$-back-and-forth relations (Definition 2.1).

Definition 3.4. For $X \subset \mathbf{A B F}_{n}$, we let $\operatorname{dc} X \subset \mathbf{A B F}_{n}$ be the $\leq_{n}$-downward closure of $X$. 
Definition 3.5. For $n>0$ an element $a \in \mathcal{A}$ is $n$-indecomposable for ultrafilter $U \ni a$ if (i) $t_{n}(U)=t_{n}(a)$ and (ii) for all $V \neq U$ with $a \in V, t_{n-1}(V) \in \operatorname{dc} t_{n}(a)$.

For $n=0$, every nonzero $a \in \mathcal{A}$ is 0-indecomposable for any ultrafilter $U \ni a$.

A Boolean algebra $\mathcal{A}$ is $n$-indecomposable for ultrafilter $U \subset \mathcal{A}$ if $1_{\mathcal{A}}$ is $n$ indecomposable for ultrafilter $U$.

We will show in the next section that the following are equivalent for a Boolean algebra $\mathcal{A}: \mathcal{A}$ is $n$-indecomposable for the ultrafilter $U ; \mathcal{A} \equiv_{n} \mathcal{A}\lceil a$ for all $a \in U$; and, for every partition $\left(a_{i}\right)_{i \leq k}$ of $\mathcal{A}$ there is some $i \leq k$ with $\mathcal{A} \equiv_{n} \mathcal{A}\left\lceil a_{i}\right.$.

In this section, when we write that $\mathcal{A}$ is $n$-indecomposable, we mean that it is $n$-indecomposable for some ultrafilter $U$.

We now define the abstract $n$-bf-types, $\mathbf{A B F}_{n}(n \in \omega)$. The model for the relation $\leq_{n}$ on $\mathbf{A B F}_{n}$ is Definition 2.1 of the $n$-back-and-forth relation on Boolean algebras and its consequence, Corollary 2.4 .

Definition 3.6. By recursion on $n$, we define a finite set $\mathbf{A B F}_{n}$ (for $n>0$, a collection of subsets of $\mathbf{A B F} \mathbf{F}_{n-1}$ ), a relation $\leq_{n}$ on the subsets of $\mathbf{A B F} \mathbf{F}_{n-1}$ (therefore a relation on $\mathbf{A B F} \mathbf{F}_{n}$ by restriction), and a map $(\cdot)_{n-1}: \mathbf{A B F} \mathbf{F}_{n} \rightarrow \mathbf{A B F} \mathbf{F}_{n-1}$. Then:

- Let $\mathbf{A B F}_{0}=\{*\}$ and $* \leq_{0} *$.

- For $\alpha \subseteq \mathbf{A B F}_{n}$, let $(\alpha)_{n}=\max \left\{(\gamma)_{n-1}: \gamma \in \alpha\right\}$ if $n>0$, and let $(\alpha)_{0}=*$.

- For $\alpha, \beta \subseteq \mathbf{A B F}_{n}$, let $\alpha \leq_{n+1} \beta$ if $(\alpha)_{n} \equiv_{n}(\beta)_{n}$ and $\forall \delta \in \beta \exists \gamma \in \alpha\left(\gamma \geq_{n}\right.$ $\delta)$.

- For $\alpha, \beta \subseteq \mathbf{A B F}_{n}$, let $\alpha \equiv_{n+1} \beta$ if $\alpha \leq_{n+1} \beta$ and $\beta \leq_{n+1} \alpha$.

- Let $\mathbf{A B F}_{n+1}$ be the set of $\leq_{n}$-antichains of $\mathbf{A B F}_{n}$.

Not all these $n$-bf-types can be realized in a Boolean algebra (hence the name abstract $n$-bf-types). We will provide conditions that describe which of the $n$-bftypes are realized in some Boolean algebra in Section 5 Later on, we will use $\mathbf{B F}_{n}$ to denote only the set of $n$-bf-types which are realized as $t_{n}(\mathcal{A})$ for some $n$ indecomposable $\mathcal{A}$. For now, we work with $\mathbf{A B F}_{n}$, the set of all abstract $n$-bf-types, realizable or not.

We introduce the following convenient notation.

Notation 3.7. Let $n \geq 1, \alpha, \beta \in \mathbf{A B F}_{n}$, and $\gamma \in \mathbf{A B F}_{n-1}$.

- We will write $\gamma \in{ }^{\mathrm{w}} \alpha$ when $\gamma \in \operatorname{dc} \alpha$ (that is, there is a $\delta \in \alpha$ with $\delta \geq_{n-1} \gamma$ ).

- We will write $\alpha \leq_{n}^{\mathrm{w}} \beta$ when $\operatorname{dc} \beta \subseteq \operatorname{dc} \alpha$ (that is, for every $\delta \in \beta$, there is a $\gamma \in \alpha$ with $\delta \leq_{n-1} \gamma$ ). We will write $\alpha<_{n}^{\mathrm{w}} \beta$ if $\alpha \leq_{n}^{\mathrm{w}} \beta$ but not $\beta \leq_{n}^{\mathrm{w}} \alpha$.

This notation will be useful because given a Boolean algebra $\mathcal{A}$ that is $n$ indecomposable for ultrafilter $U$ and $V \in \operatorname{Ult}(\mathcal{A})$ with $V \neq U$, both $t_{n-1}(V) \in^{\mathrm{w}}$ $t_{n}(\mathcal{A})$ and $t_{n}(\mathcal{A}) \leq_{n}^{\mathrm{w}} t_{n}(V)$ hold. (This is a consequence of Lemma 3.10, )

Note that if $\alpha, \beta \subseteq \mathbf{A B F}_{n}$ are $\leq_{n}$-antichains and $\alpha \equiv_{n+1} \beta$, then $\alpha=\beta$. The reason is that if $\gamma \in \alpha$, then there is a $\delta \in \beta$ and $\xi \in \alpha$ so that so that $\gamma \leq_{n} \delta \leq_{n} \xi$. Thus, $\gamma=\xi=\delta$ by the maximality of $\gamma$, and so $\alpha \subseteq \beta$. Similarly, $\beta \subseteq \alpha$.

As in the definitions above, in the next few lemmas it could be useful for the reader to keep in mind the Stone space $\operatorname{Ult}(\mathcal{A})$ of the Boolean algebra being considered. For example, if $U \in \operatorname{Ult}(\mathcal{A})$ and $a, b \in \mathcal{A}$, one could read $a \in U$ as $U \in \mathcal{O}_{a}$ and $a \leq b$ as $\mathcal{O}_{a} \subseteq \mathcal{O}_{b}$.

3.2. Proof of compactness. This subsection and the next are dedicated to showing that $\leq_{n}, t_{n}(\cdot),(\cdot)_{n-1}$, and $n$-indecomposables have the properties claimed. The 
proofs are mostly combinatorial and not very hard. We recommend the reader to read the statements and comments and to skip the proofs in a first read of the paper.

Lemma 3.8. Let $n \geq 0$ and $\alpha, \beta \in \mathbf{A B F}_{n+1}$. Then:

(a) $\alpha \leq_{n+1} \beta$ if and only if $\alpha \leq_{n+1}^{\mathrm{w}} \beta$ and $(\alpha)_{n} \equiv_{n}(\beta)_{n}$.

(b) $\alpha \leq_{n+1}^{\mathrm{w}} \beta$ implies $(\alpha)_{n} \leq_{n}^{\mathrm{w}}(\beta)_{n}$.

(c) $\alpha \equiv_{n+1} \beta$ if and only if $\alpha \leq_{n+1}^{\mathrm{w}} \beta$ and $\beta \leq_{n+1}^{\mathrm{w}} \alpha$.

(d) $\alpha \leq_{n+1} \beta$ if and only if $(\alpha)_{n} \geq_{n}^{\mathrm{W}}(\beta)_{n}$ and $\alpha \leq_{n+1}^{\mathrm{w}} \beta$.

It follows from (드) that every $\alpha \subseteq \mathbf{A B F}_{n}$ is $\equiv_{n+1}$-equivalent to the antichain of its $\leq_{n}$-maximal elements.

Proof. (国) This follows from the definition of $\leq_{n+1}$.

(b) Pick $\delta \in(\beta)_{n}$ and let $\delta^{\prime} \in \beta$ be such that $\left(\delta^{\prime}\right)_{n-1}=\delta$. By hypothesis there is a $\gamma \in \alpha$ with $\delta^{\prime} \leq_{n} \gamma$. Therefore, $\delta=\left(\delta^{\prime}\right)_{n-1} \equiv_{n-1}(\gamma)_{n-1} \in \in^{\mathrm{w}}(\alpha)_{n}$. Since $\delta$ was arbitrary, $(\alpha)_{n} \leq_{n}^{\mathrm{w}}(\beta)_{n}$.

(ㄷ) The direction $(\Rightarrow)$ follows from (国). We now show $(\Leftarrow)$. Assume for $\alpha, \beta \subseteq$ $\mathbf{A B F}_{n}$ that $\beta \leq_{n+1}^{\mathrm{w}} \alpha$ and $\alpha \leq_{n+1}^{\mathrm{w}} \beta$. By (b) we have that $(\alpha)_{n}=(\beta)_{n}$. Then, by (国), we have that $\beta \leq_{n+1} \alpha$ and $\alpha \leq_{n+1} \beta$ as wanted. (dd) The direction $(\Rightarrow)$ follows from (国). For the other direction, by (b), $(\alpha)_{n} \leq_{n}^{\mathrm{w}}(\beta)_{n}$, and then by (ㄷ) and hypothesis, $(\alpha)_{n} \equiv_{n}(\beta)_{n}$. Then, use (国) again.

The following facts are easily verified and will be appealed to without comment:

- $a \leq b$ implies $t_{n}(b) \leq_{n}^{\mathrm{w}} t_{n}(a)$.

- $a=a_{0} \vee \ldots \vee a_{k}$ implies $t_{n}(a) \equiv_{n} \max \bigcup_{i} t_{n}\left(a_{i}\right)$.

The assignments $t_{n}$ respect the operation $(\cdot)_{n-1}$.

Lemma 3.9. Let $\mathcal{A}$ be any Boolean algebra. Then:

(1) $t_{n}(U) \equiv_{n}\left(t_{n+1}(U)\right)_{n}$, for every $U \in \operatorname{Ult} \mathcal{A}$.

(2) $t_{n}(a) \equiv_{n}\left(t_{n+1}(a)\right)_{n}$, for every $a \in \mathcal{A}$.

Proof. (11) The proof is by induction, where the basis case $n=0$ is trivial. Assume it is true for $n-1$. We show (i) $t_{n}(U) \leq_{n}^{\mathrm{w}}\left(t_{n+1}(U)\right)_{n}$ and (ii) $\left(t_{n+1}(U)\right)_{n} \leq_{n}^{\mathrm{w}} t_{n}(U)$. The conclusion follows by Lemma 3.8(C).

For (i), suppose that $\alpha \in t_{n+1}(U)$ with $(\alpha)_{n-1} \in\left(t_{n+1}(U)\right)_{n}$. Then for any $a \in U$ there is an ultrafilter $V \ni a$ distinct from $U$ with $t_{n}(V)=\alpha$. By the inductive hypothesis, $t_{n-1}(V) \equiv_{n-1}\left(t_{n}(V)\right)_{n-1}=(\alpha)_{n-1}$, and hence $(\alpha)_{n-1} \in$ $\hat{t}_{n}(U)$. Therefore, by the definition of $t_{n}(U)$, there is a $\gamma \in t_{n}(U)$ with $(\alpha)_{n-1} \leq_{n-1}$ $\gamma$. We conclude that $t_{n}(U) \leq_{n}^{\mathrm{w}}\left(t_{n+1}(U)\right)_{n}$.

For (ii), we will prove the stronger condition that $t_{n}(U) \subseteq\left(t_{n+1}(U)\right)_{n}$. Let $\alpha \in$ $t_{n}(U)$ and suppose for reductio that there is no $\beta \in t_{n+1}(U)$ with $(\beta)_{n-1} \equiv_{n-1} \alpha$. That is, for each $\beta \in \mathbf{A B F}_{n}$ with $(\beta)_{n-1} \equiv_{n-1} \alpha$, there is $a_{\beta} \in U$ such that no $V \ni a_{\beta}$ distinct from $U$ satisfies $t_{n}(V)=\beta$. Since $\mathbf{A} \mathbf{B} \mathbf{F}_{n}$ is finite, list the $n$-bf-types with $(\beta)_{n-1} \equiv_{n-1} \alpha$ as $\left\{\beta_{0}, \ldots, \beta_{\ell}\right\}$, and choose witnesses $\left\{a_{\beta_{0}}, \ldots, a_{\beta_{\ell}}\right\}$ as by the reductio supposition. Let $a=\bigwedge_{i \leq \ell} a_{\beta_{i}}$, so that $a \in U$, and let $V \ni a$ be distinct from $U$ with $t_{n-1}(V)=\alpha$. Then by the inductive hypothesis, $\left(t_{n}(V)\right)_{n-1}=\alpha$, so that $t_{n}(V)=\beta_{i}$ (for some $i \leq \ell$ ). However, then $a_{\beta_{i}} \in V$, contradicting the choice of $a_{\beta_{i}}$. Thus, for some $\beta \in \mathbf{A B F}_{n}$ with $(\beta)_{n-1} \equiv_{n-1} \alpha$ we have $\beta \in t_{n+1}(U)$. 
(2) We will show (i) $t_{n}(a) \leq_{n}^{\mathrm{w}}\left(t_{n+1}(a)\right)_{n}$ and (ii) $\left(t_{n+1}(a)\right)_{n} \leq_{n}^{\mathrm{w}} t_{n}(a)$. The conclusion follows by Lemma 3.8(c).

For (i), suppose $\alpha \in t_{n+1}(a)$ with $(\alpha)_{n-1} \in\left(t_{n+1}(a)\right)_{n}$. Then there are infinitely many ultrafilters $V \ni a$ with $t_{n}(V)=\alpha$. By (10), for each such $V, t_{n-1}(V) \equiv_{n-1}$ $\left(t_{n}(V)\right)_{n-1}$, so that for some $\gamma \in t_{n}(a),(\alpha)_{n-1} \leq_{n-1} \gamma$.

For (ii), suppose $\alpha \in t_{n}(a)$. Since $\mathbf{A B F}_{n}$ is finite, there is a $\beta \in \mathbf{A} \mathbf{B} \mathbf{F}_{n}$ with infinitely many ultrafilters $V \ni a$ satisfying $t_{n}(V)=\beta$ and $(\beta)_{n-1} \equiv_{n-1} \alpha$. Therefore, there is a $\gamma \in t_{n+1}(a)$ with $\beta \leq_{n} \gamma$, and thus $(\gamma)_{n-1} \equiv_{n-1}(\beta)_{n-1} \equiv_{n-1} \alpha$.

The next three lemmas are dedicated to proving that for every ultrafilter $U$ of $\mathcal{A}$ and for every $n$, there exists $a \in U$ such that $a$ is $n$-indecomposable for $U$ (Lemma 3.12(1)).

Lemma 3.10. Let $n>0$. Then for every ultrafilter $U$ and every $a \in U, t_{n}(a) \leq_{n}^{\mathrm{w}}$ $t_{n}(U)$. In particular, if $\mathcal{A}$ is n-indecomposable for ultrafilter $U$ and $V \in \operatorname{Ult}(\mathcal{A})$ is distinct from $U$, then $t_{n-1}(V) \in{ }^{\mathrm{w}} t_{n}(\mathcal{A})$ and $t_{n}(\mathcal{A}) \leq_{n}^{\mathrm{w}} t_{n}(V)$.

Note that $a \in U$ does not imply that $t_{n}(a)=t_{n}(U)$.

Proof. Fix $a$, an ultrafilter $U \ni a$, and let $\alpha \in t_{n}(U)$. We argue by induction that for each $k \in \omega$ there are at least $k$ ultrafilters containing $a$ whose $(n-1)$-bf-type is $\alpha$ and that are each distinct from $U$. Given $k$ such ultrafilters $V_{0}, \ldots, V_{k-1}$, there is a $b<a$ with $b \in U-V_{i}$ for all $i<k$. By the definition of $t_{n}(U)$, there is an ultrafilter $V_{k}$ distinct from $U$ with $b \in V_{k}$ and $t_{n-1}\left(V_{k}\right)=\alpha$. Thus, we have $a \in V_{k}$, and $V_{k}$ is distinct from $U$ and each $V_{i}(i<k)$. This completes the induction. From the definition of $t_{n}(a)$, there is a $\gamma \in t_{n}(a)$ such that $\alpha \leq_{n-1} \gamma$.

For the second part of the lemma, note that $t_{n-1}(V) \in{ }^{\mathrm{w}} t_{n}(\mathcal{A})$ by Definition 3.5 and $t_{n}(\mathcal{A}) \leq_{n}^{\mathrm{w}} t_{n}(V)$ by the first part of the lemma, since $1_{\mathcal{A}} \in V$.

Lemma 3.11. For every ultrafilter $U$, there is an $a \in U$ such that $t_{n}(U)=t_{n}(a)$.

Proof. Let $U$ be an ultrafilter for the Boolean algebra $\mathcal{A}$. We show that if $a \in U$ and $t_{n}(a)<_{n}^{\mathrm{w}} t_{n}(U)$, then there is a $b \in U$ with $b<a$ and $t_{n}(a)<_{n}^{\mathrm{w}} t_{n}(b) \leq_{n}^{\mathrm{w}} t_{n}(U)$. The lemma follows as $t_{n}\left(1_{\mathcal{A}}\right) \leq_{n}^{\mathrm{w}} t_{n}(U)$ (by Lemma 3.10) and $t_{n}\left(1_{\mathcal{A}}\right)$ is a finite set.

Suppose $a \in U$ and $t_{n}(a)<_{n}^{\mathrm{W}} t_{n}(U)$; let $\alpha \in t_{n}(a)$ but no $\gamma \in t_{n}(U)$ satisfy $\alpha \leq_{n-1} \gamma$. For each $\gamma \geq_{n-1} \alpha$ there is a $c_{\gamma} \in U$ for which there is no ultrafilter $V \ni c_{\gamma}$ distinct from $U$ with $t_{n-1}(V)=\gamma$. Let $I=\left\{\gamma: \gamma \geq_{n-1} \alpha\right\}$ and $b=$ $a \wedge \bigwedge_{\gamma \in I} c_{\gamma}$. Therefore, $b \in U$, and for no $\gamma \geq_{n-1} \alpha$ is it the case that $\gamma \in t_{n}(b)$. Thus, $t_{n}(a)<_{n}^{\mathrm{w}} t_{n}(b) \leq_{n}^{\mathrm{w}} t_{n}(U)$.

Lemma 3.12. (1) For every ultrafilter $U$, there is an $a \in U$ which is $n$ indecomposable for $U$.

(2) If $a$ is $n$-indecomposable for an ultrafilter $U$, then every $b \in U \uparrow a$ is also $n$-indecomposable for $U$.

Proof. (11) Let $U$ be an ultrafilter. By Lemma 3.11 there exists $b \in U$ with $t_{n}(b)=$ $t_{n}(U)$. For each $\alpha \in \mathbf{A B F}_{n-1}$, let $k_{\alpha}$ be the number of ultrafilters (possibly infinite) $V \neq U$ with $b \in V$ and $\alpha=t_{n-1}(V)$. Since $t_{n}(b)=t_{n}(U)$, for every $\alpha \notin^{\mathrm{w}} t_{n}(U), k_{\alpha}$ is finite. Let $\mathcal{U}=\left\{V \in \operatorname{Ult}(\mathcal{A}): b \in V \& V \neq U \& t_{n-1}(V) \notin^{\mathrm{w}} t_{n}(U)\right\}$. The set $\mathcal{U}$ is finite. Therefore, there exists $a \leq b$ with $a \in U$ but $a \notin V$ for any $V \in \mathcal{U}$. Since $t_{n}(b) \leq_{n}^{\mathrm{w}} t_{n}(a) \leq_{n}^{\mathrm{w}} t_{n}(U)=t_{n}(b)$, we have that $t_{n}(a)=t_{n}(U)$. Also, for any $V$ with $a \in V$, since $V \notin \mathcal{U}, t_{n-1}(V) \in{ }^{\mathrm{w}} t_{n}(U)$. It follows that $a$ is $n$-indecomposable for $U$. 
(2) Let $a$ be $n$-indecomposable for ultrafilter $U$ and $b \in U \uparrow a$. We show that $b$ satisfies conditions (i) and (ii) of Definition 3.5 for $U$. Since $b \leq a, t_{n}(U)=$ $t_{n}(a) \leq_{n}^{\mathrm{w}} t_{n}(b) \leq_{n}^{\mathrm{w}} t_{n}(U)$, so $t_{n}(U)=t_{n}(b)$ and (i) holds. Since (ii) holds for $a$, $b \leq a$ and $t_{n}(a)=t_{n}(b)$, (ii) holds for $b$.

From now on we will only apply the map $t_{n}$ to $n$-indecomposable algebras.

The following compactness theorem follows from the compactness of $\operatorname{Ult}(\mathcal{A})$ and plays a key role in the development that follows.

Theorem 3.13. For every $n \in \omega$ and every Boolean algebra $\mathcal{A}$, there is a partition $\left(a_{i}\right)_{i \leq k}$ of $\mathcal{A}$ where each $a_{i}$ is $n$-indecomposable.

Proof. For each ultrafilter $U$, fix $a_{U} \in U$ which is $n$-indecomposable for $U$. Since $\operatorname{Ult}(\mathcal{A})$ is a compact topological space, the open covering $\left\{\mathcal{O}_{a_{U}}: U \in \operatorname{Ult}(\mathcal{A})\right\}$ has a finite subcovering. We include a proof of this: Suppose, toward a contradiction, that for every finite set $\left\{a_{U_{0}}, \ldots, a_{U_{k}}\right\}$, we have $1_{\mathcal{A}} \neq a_{U_{0}} \vee \ldots \vee a_{U_{k}}$. Therefore, $0_{\mathcal{A}} \neq-a_{U_{0}} \wedge \ldots \wedge-a_{U_{k}}$. Thus, there is a (proper) ultrafilter $V$ extending the set $\left\{-a_{U}: U \in\right.$ Ult $\left.\mathcal{A}\right\}$ (see [Mon89, Proposition 2.16]). However, in this case, $a_{V},-a_{V} \in V$, which contradicts the fact that $V$ is proper.

Thus, there are $n$-indecomposable elements $a_{U_{0}}, \ldots, a_{U_{\ell}}$ for filters $U_{0}, \ldots, U_{\ell}$ such that $1_{\mathcal{A}}=a_{U_{0}} \vee \ldots \vee a_{U_{\ell}}$. Using Lemma 3.12(2) we may refine $\left(a_{U_{i}}\right)_{i \leq \ell}$ to a partition of $\mathcal{A}$ as required.

3.3. Proof of invariance. Now our goal is to show that for $n$-indecomposable Boolean algebras $\mathcal{A}$ and $\mathcal{B}, \mathcal{A} \leq_{n} \mathcal{B}$ if and only if $t_{n}(\mathcal{A}) \leq_{n} t_{n}(\mathcal{B})$.

The following lemma refines the definition of the $n$-back-and-forth relations.

Lemma 3.14. For all Boolean algebras $\mathcal{A}$ and $\mathcal{B}$ and for every $n \in \omega, \mathcal{A} \leq_{n+1} \mathcal{B}$ if and only if for every partition $\left(b_{i}\right)_{i \leq k}$ of $\mathcal{B}$ into $n$-indecomposables, there is a partition $\left(a_{i}\right)_{i \leq k}$ of $\mathcal{A}$ such that $\left(b_{i}\right)_{i \leq k} \leq_{n}\left(a_{i}\right)_{i \leq k}$.

Note that there is no claim that the partition of $\mathcal{A}$ given by the theorem is into $n$-indecomposables. Indeed, this may not be possible (see Example 7.21).

Proof. $(\Rightarrow)$ follows from Definition 2.1 . For $(\Leftarrow)$ : given any partition $\left(b_{i}\right)_{i \leq k}$, we can refine this partition into a partition of $n$-indecomposables using Theorem 3.13 on $\mathcal{B} \uparrow b_{i}$; then, apply Lemma 2.2 to the matching partition from $\mathcal{A}$.

Lemma 3.15. Let a be $(n+1)$-indecomposable for ultrafilter $U$ and $\alpha \in \mathbf{A B F}_{n}$.

(1) If $\alpha \in \in^{\mathrm{w}} t_{n+1}(a)$, then for any $k \in \omega$ there is a partition $\left(a_{i}\right)_{i \leq k}$ of a with $a_{0} \in U$ and $\alpha \leq_{n} t_{n}\left(a_{i}\right)$ (for $\left.1 \leq i \leq k\right)$, where each $a_{i}$ is $n$-indecomposable for some ultrafilter.

(2) If $\left(a_{i}\right)_{i \leq k}$ is a partition of a with $a_{0} \in U$ and each $a_{i}(1 \leq i \leq k)$ is $n$ indecomposable for some ultrafilter, then $t_{n}\left(a_{i}\right) \in{ }^{\mathrm{w}} t_{n+1}(a)$ for each $1 \leq i \leq$ $k$.

Proof. (11) Suppose that $\alpha \in \in^{\mathrm{w}} t_{n+1}(a)$ so that for some $\gamma \in \mathbf{A B F}_{n}, \alpha \leq n \gamma \in$ $t_{n+1}(a)$. Then there are distinct ultrafilters $V_{1}, \ldots, V_{k}$, all distinct from $U$, with $a \in V_{i}$ and $\gamma=t_{n}\left(a_{i}\right)$ for $i \leq k$ (see the proof of Lemma 3.10). Fix $a_{1}, \ldots, a_{k}<a$ such that $a_{i}$ is $n$-indecomposable for $V_{i}$ but $a_{i} \notin U, V_{j}$ for any $j \neq i$. Moreover, choose the $a_{i}$ to be pairwise disjoint. Let $a_{0}=a-\left(a_{1} \vee \ldots \vee a_{k}\right)$; then $a_{0} \in U$.

(2) Let $a=a_{0} \dot{V} \ldots \dot{\vee} a_{k}$ where $a_{0} \in U$ and each $a_{i}(1 \leq i \leq k)$ is $n$ indecomposable for some ultrafilter $V_{i}$. Then $V_{i} \neq U$ and $a \in V_{i}$ so that $t_{n}\left(V_{i}\right) \in \in^{\mathrm{w}}$ $t_{n+1}(a)$ by condition (ii) of Definition 3.5. Thus, $t_{n}\left(a_{i}\right) \in{ }^{\mathrm{w}} t_{n+1}(a)$, for $i \geq 1$. 
The following gives one-half of a back-and-forth characterization of when $n$ indecomposable algebras have the same $n$-bf-type (the converse follows by Theorem 3.17 and Lemma 4.3).

Lemma 3.16. Let $n>0$ and $\mathcal{A}$ and $\mathcal{B}$ be $n$-indecomposable for ultrafilters $U$ and $V$, respectively. If $t_{n}(\mathcal{B}) \leq_{n} t_{n}(\mathcal{A})$, then for every partition $\left(a_{i}\right)_{i \leq k}$ of $\mathcal{A}$ into $(n-1)$-indecomposable elements, there is a partition $\left(b_{i}\right)_{i \leq k}$ of $\mathcal{B}$ into $(n-1)$ indecomposable elements with $t_{n-1}\left(a_{i}\right) \leq_{n-1} t_{n-1}\left(b_{i}\right)$ for each $i \leq k$.

Proof. Suppose that $t_{n}(\mathcal{B}) \leq_{n} t_{n}(\mathcal{A})$. Note that $t_{n}(V) \leq_{n} t_{n}(U)$ and $t_{n}\left(1_{\mathcal{B}}\right) \leq_{n}$ $t_{n}\left(1_{\mathcal{A}}\right)$. The proof is by induction on the length of the partition $k$, where $k=0$ holds by supposition. Suppose for induction that

for any partition $\left(c_{i}\right)_{i \leq k-1}$ of $\mathcal{A}$ where $c_{0} \in U$ and each $c_{i}$ is $(n-1)$ indecomposable, there is a partition $\left(d_{i}\right)_{i \leq k-1}$ of $\mathcal{B}$ where $d_{0} \in V$, each $d_{i}$ is $(n-1)$-indecomposable, and such that $t_{n-1}\left(c_{i}\right) \leq_{n-1} t_{n-1}\left(d_{i}\right)$ for each $i<k$.

Consider any partition $\left(a_{i}\right)_{i \leq k}$ of $\mathcal{A}$, where $a_{0} \in U$ and each $a_{i}$ is $(n-1)$ indecomposable. Then, $a_{0} \vee a_{k} \in U$ and is $n$-indecomposable for $U$ (since $1_{\mathcal{A}}$ is $n$-indecomposable for $U$ by Lemma 3.12(2) ). By the inductive hypothesis there is a matching partition of $\mathcal{B},\left(b_{i}\right)_{i<k-1}$, where $b_{0} \in V$ (and so is $n$-indecomposable by Lemma 3.12(2) , each $b_{i}$ is $(n-1)$-indecomposable, and such that $t_{n-1}\left(a_{i}\right) \leq_{n-1}$ $t_{n-1}\left(b_{i}\right)$ for all $i<k$. Since $b_{0} \in V$ and $\left(a_{0} \vee a_{k}\right) \in U$ and both are $n$-indecomposable, it follows that $t_{n}\left(b_{0}\right) \leq_{n} t_{n}\left(a_{0} \vee a_{k}\right)$.

As $a_{k}$ is $(n-1)$-indecomposable, let $U_{k}$ be an ultrafilter for which it is $(n-1)$ indecomposable, and let $\alpha=t_{n-1}\left(a_{k}\right)=t_{n-1}\left(U_{k}\right)$. Since $a_{0} \vee a_{k}$ is $n$-indecomposable, there exists some $\gamma \in t_{n}\left(a_{0} \vee a_{k}\right)$ with $\alpha \leq_{n-1} \gamma$. However, then there is a $\delta \in$ $t_{n}\left(b_{0}\right)$ with $\gamma \leq_{n-1} \delta$, so that there is an ultrafilter $V_{k} \ni b_{0}$ distinct from $V$ with $t_{n-1}\left(V_{k}\right)=\delta$. Partition $b_{0}$ as $b_{0}^{\prime} \dot{\vee} b_{k}$, where $b_{k}$ is $(n-1)$-indecomposable for $V_{k}$ and $b_{0}^{\prime} \in V$. Thus, we have $t_{n-1}\left(a_{k}\right) \leq_{n-1} t_{n-1}\left(b_{k}\right)$ and $t_{n-1}\left(a_{0}\right) \equiv_{n-1} t_{n-1}\left(b_{0}^{\prime}\right)$.

We arrive at the main result of this section.

Theorem 3.17. Let $\mathcal{A}$ and $\mathcal{B}$ be $n$-indecomposable Boolean algebras. Then

$$
\mathcal{A} \leq_{n} \mathcal{B} \quad \Longleftrightarrow \quad t_{n}(\mathcal{A}) \leq_{n} t_{n}(\mathcal{B})
$$

Proof. The proof is by induction on $n$. We will simultaneously prove the following result, which will be of independent interest.

Lemma 3.18. Let $a \in \mathcal{A}$ be $n$-indecomposable and $d_{0}, \ldots, d_{k} \in \mathcal{B}$ satisfy $a \leq_{n}$ $\bigvee_{i \leq k} d_{i}$. Then $a \leq{ }_{n} d_{i}$ for some $i \leq k$.

When $n=0$ the lemma and the theorem hold trivially. We assume the theorem holds for $n-1$, and we will later show that this implies that the lemma holds for $n$. First, we use that the lemma holds for $n$ to prove the theorem for $n$.

$(\Leftarrow)$. Suppose $t_{n}(\mathcal{A}) \leq_{n} t_{n}(\mathcal{B})$. To prove $\mathcal{A} \leq_{n} \mathcal{B}$, consider any partition of $\mathcal{B},\left(b_{i}\right)_{i \leq k}$, into $(n-1)$-indecomposables. (This is sufficient by Lemma 3.14.) By Lemma 3.16 there is a matching partition of $\mathcal{A},\left(a_{i}\right)_{i \leq k}$, into $(n-1)$-indecomposables such that $t_{n-1}\left(b_{i}\right) \leq_{n-1} t_{n-1}\left(a_{i}\right)$ (for each $\left.i \leq k\right)$. By the inductive hypothesis, $\left(b_{i}\right)_{i \leq k} \leq_{n-1}\left(a_{i}\right)_{i \leq k}$.

$(\Rightarrow)$. Suppose $\mathcal{A} \leq_{n} \mathcal{B}$, where $\mathcal{A}$ is $n$-indecomposable for ultrafilter $U$ and $\mathcal{B}$ is $n$ indecomposable for ultrafilter $V$. Then, $\mathcal{A} \equiv_{n-1} \mathcal{B}$, so by the inductive hypothesis, 
$t_{n-1}(\mathcal{A}) \equiv_{n-1} t_{n-1}(\mathcal{B})$; thus, $\left(t_{n}(\mathcal{A})\right)_{n-1} \equiv_{n-1}\left(t_{n}(\mathcal{B})\right)_{n-1}$ by Lemma 3.9]2). To conclude $t_{n}(\mathcal{A}) \leq_{n} t_{n}(\mathcal{B})$, it remains to show $t_{n}(\mathcal{A}) \leq_{n}^{\mathrm{w}} t_{n}(\mathcal{B})$. Let $\beta \in t_{n}(\mathcal{B})$. There is a partition $\left(b_{0}, b_{1}, b_{2}\right)$ of $\mathcal{B}$ into $(n-1)$-indecomposables with $b_{0} \in V$ and $t_{n-1}\left(b_{i}\right) \geq_{n-1} \beta$ (for $i \in\{1,2\}$ ) by Lemma 3.15(1). Since $\beta \in t_{n}(\mathcal{B})$ we must actually have $t_{n-1}\left(b_{i}\right)=\beta$ (for $i \in\{1,2\}$ ). There is a matching partition $\left(a_{0}, a_{1}, a_{2}\right)$ of $\mathcal{A}$ with $b_{i} \leq_{n-1} a_{i}$ for $i \in\{0,1,2\}$. Since $\mathcal{A}$ is $n$-indecomposable for $U$, there is some $i \leq 2$ such that $a_{i}$ is $n$-indecomposable for $U$; for this $i, a_{0} \vee a_{i} \in U$, so that $a_{0} \vee a_{i} \equiv_{n} a_{i} \equiv_{n} 1_{\mathcal{A}}$. We also have $b_{0} \vee b_{i} \in V$, so that $b_{0} \vee b_{i} \equiv_{n} b_{0} \equiv_{n} 1_{\mathcal{B}}$. Thus, $b_{0} \vee b_{i} \equiv_{n-1} a_{0} \vee a_{i}$. In what follows we will consider a partition $\left(b_{0}, b_{1}\right)$ of $\mathcal{B}$ with $b_{0} \in V$ and $t_{n-1}\left(b_{1}\right)=\beta$, and a partition $\left(a_{0}, a_{1}\right)$ of $\mathcal{A}$ with $a_{0} \in U$ and $b_{i} \leq_{n-1} a_{i}$ for $i \in\{0,1\}$.

We complete the proof assuming Lemma 3.18 holds for $n-1$. Partition $a_{1}$ further into $(n-1)$-indecomposables, $\left(a_{j}^{\prime}\right)_{j \leq m}$, so that $a_{1}=\bigvee_{j \leq m} a_{j}^{\prime}$. Since $b_{1}$ is $(n-1)$ indecomposable for some ultrafilter and $b_{1} \leq_{n-1} \bigvee_{j \leq m} a_{j}^{\prime}$, it follows by Lemma 3.18 that $b_{1} \leq_{n-1} a_{j}^{\prime}$ (for some $j \leq m$ ), and thus by the inductive hypothesis, $t_{n-1}\left(b_{1}\right) \leq_{n-1} t_{n-1}\left(a_{j}^{\prime}\right)$. Since $\beta=t_{n-1}\left(b_{1}\right) \leq_{n-1} t_{n-1}\left(a_{j}^{\prime}\right)$ and $a_{j}^{\prime}$ is $(n-1)$ indecomposable, it follows by Lemma 3.15)(2) that $\beta \leq_{n-1} \gamma$ for some $\gamma \in t_{n}(\mathcal{A})$. Since $\beta \in t_{n}(\mathcal{B})$ was arbitrary, it follows that $t_{n}(\mathcal{A}) \leq_{n}^{\mathrm{w}} t_{n}(\mathcal{B})$.

Proof of Lemma 3.18. We assume Theorem 3.17holds for $n-1$ and show the lemma for $n$.

We prove the following special case: for any $a \in \mathcal{A}$ which is $n$-indecomposable (for ultrafilter $U$ ) and partition $\left(b_{0}, b_{1}\right)$ of $\mathcal{B}$, if $a \leq_{n} b_{0} \vee b_{1}$, then $a \leq_{n} b_{0}$ or $a \leq_{n} b_{1}$. The lemma then follows by induction using this special case.

Suppose $a \mathbb{Z}_{n} b_{0}$ and let $\left(d_{i}\right)_{i \leq k}$ be a partition of $b_{0}$ with no matching partition $\left(a_{i}\right)_{i \leq k}$ of $a$ with $\left(a_{i}\right)_{i \leq k} \geq_{n-1}\left(d_{i}\right)_{i \leq k}$. To show that $a \leq_{n} b_{1}$, consider any partition $\left(e_{j}\right)_{j \leq \ell}$ of $b_{1}$ so that by hypothesis there is a partition $\left(a_{i}\right)_{i \leq k+\ell}$ such that $\left(d_{i}\right)_{i \leq k} \leq_{n-1}\left(a_{i}\right)_{i \leq k}$ and $\left(e_{i}\right)_{i \leq \ell} \leq_{n-1}\left(a_{k+i}\right)_{i \leq \ell}$. Since $a$ is $n$-indecomposable for ultrafilter $U$, there is some $i \leq k+\ell$ with $a_{i} \in U$ and that is $n$-indecomposable for $U$. We will show that $i>k$. Suppose that $i \leq k$, say $a_{0} \in U$. Then let $d=a_{0} \dot{V} a_{k+1} \dot{V} \ldots \dot{V} a_{k+\ell} \in U$, which is $n$-indecomposable for $U$. Thus, $t_{n-1}\left(a_{0}\right)=t_{n-1}(U)=t_{n-1}(d)$ so that $a_{0} \equiv_{n-1} d$ by the inductive hypothesis for Theorem 3.17 and $\left(d, a_{1}, \ldots, a_{k}\right)$ is a partition of $a$ satisfying $\left(d, a_{1}, \ldots, a_{k}\right) \geq_{n-1}$ $\left(d_{i}\right)_{i \leq k}$, contradicting our assumption about the partition $\left(d_{i}\right)_{i \leq k}$ of $b_{0}$. Therefore, we may assume $a_{k+1}$ is $n$-indecomposable for $U$. Let $e=a_{k+1} \dot{\vee} a_{0} \dot{V} \ldots \dot{\vee} a_{k}$ so, $e$ is $n$-indecomposable for $U$ and $a_{k+1} \equiv_{n-1} e$. Thus, $\left(e, a_{k+2}, \ldots, a_{k+\ell}\right)$ is a partition of $a$ with $\left(e_{j}\right)_{j \leq \ell} \leq_{n-1}\left(e, a_{k+2}, \ldots, a_{k+\ell}\right)$. Since the partition $\left(e_{j}\right)_{j \leq \ell}$ of $b_{1}$ was arbitrary, it follows that $a \leq_{n} b_{1}$.

This completes the proof of Theorem 3.17

\section{An Alternative CharaCterization of $n$-IndeCOMPosable}

We provide an equivalence between our heuristic definition of $n$-indecomposable types (Definition 3.1) and our definition in terms of accumulation points in the Stone space (Definition [3.5). Along the way we prove some useful properties of $n$-indecomposables. Our main result here is:

Theorem 4.1. For any Boolean algebra $\mathcal{A}$ the following are equivalent:

(1) $\mathcal{A}$ is $n$-indecomposable for an ultrafilter $U$ (as in Definition 3.5 ). 
(2) There is an ultrafilter $U$ such that $\mathcal{A} \equiv_{n} \mathcal{A}\lceil$ a for all $a \in U$.

(3) For every partition $\left(a_{i}\right)_{i \leq k}$ of $\mathcal{A}$ there is some $i \leq k$ with $\mathcal{A} \equiv_{n} \mathcal{A}\left\lceil a_{i}\right.$ (as in Definition 3.1).

We first prove (11) $\Rightarrow$ (2).

Lemma 4.2. For any Boolean algebra $\mathcal{A}$, if $\mathcal{A}$ is n-indecomposable for an ultrafilter $U$, then $\mathcal{A} \equiv_{n} \mathcal{A}\lceil$ a for all $a \in U$.

Proof. If $\mathcal{A}$ is $n$-indecomposable for $U$, then for all $a \in U, t_{n}(\mathcal{A}) \equiv_{n} t_{n}(a)$ and $a$ is also $n$-indecomposable for $U$ by Lemma 3.12(2). By Theorem 3.17, $\mathcal{A} \equiv_{n} \mathcal{A}\lceil a$ for all $a \in U$.

Lemma 4.3. For all Boolean algebras $\mathcal{A}$ and $\mathcal{B}$ and for every $n \in \omega$, if $\mathcal{A}$ is $(n+1)$-indecomposable for ultrafilter $U$, then $\mathcal{A} \leq_{n+1} \mathcal{B}$ if and only if for every partition $\left(b_{i}\right)_{i \leq k}$ of $\mathcal{B}$ into $n$-indecomposables, there is a partition of $\left(a_{i}\right)_{i \leq k}$ of $\mathcal{A}$ into $n$-indecomposables satisfying $\left(b_{i}\right)_{i \leq k} \leq_{n}\left(a_{i}\right)_{i \leq k}$.

Note that we impose no condition on $\mathcal{B}$ as we did in Lemma 3.16.

Proof. $(\Leftarrow)$. This follows by Lemma 3.14 .

$(\Rightarrow)$. Suppose $\mathcal{A}$ is $(n+1)$-indecomposable for ultrafilter $U$ and $\mathcal{A} \leq_{n+1} \mathcal{B}$. Let $\left(b_{i}\right)_{i \leq k}$ be a partition of $\mathcal{B}$ into $n$-indecomposables. Since $\mathcal{A}$ is $(n+1)$-indecomposable, by Lemma 3.18 we may assume that $\mathcal{A} \leq_{n+1} \mathcal{B} \uparrow b_{0}$, which implies $\mathcal{A} \equiv_{n} \mathcal{B} \uparrow$ $b_{0}$.

By hypothesis, there is a partition $\left(a_{i}\right)_{i \leq k}$ of $\mathcal{A}$ satisfying $\left(b_{i}\right)_{i \leq k} \leq_{n}\left(a_{i}\right)_{i \leq k}$, where the $a_{i}$ may not be $n$-indecomposable. Partition each $a_{i}$ into $n$-indecomposables, $\left(a_{i, j}\right)_{j \leq m_{i}}$ (by Theorem 3.13). We will also assume $b_{i} \leq_{n} a_{i, 0}$ (for each $i \leq k$ ) by Lemma 3.18 Since $\mathcal{A}$ is $(n+1)$-indecomposable, by Lemma 4.2 there is some $a_{i, j} \in U$ so that $\mathcal{A} \equiv_{n} \mathcal{A}\left\lceil a_{i, j}\right.$. If $j=0$, then let $a_{i, 0}^{\prime}=a_{i, 0} \vee \bigvee_{\ell \leq k} \bigvee_{1 \leq j \leq m_{\ell}} a_{\ell, j}$ so that $a_{i, 0}^{\prime} \in U$ and $b_{i} \leq_{n} a_{i, 0} \equiv_{n} a_{i, 0}^{\prime}$. Otherwise, let $a_{0,0}^{\prime}=a_{0,0} \vee \bigvee_{\ell \leq k} \bigvee_{1 \leq j \leq m_{\ell}} a_{\ell, j}$, so that $a_{0,0}^{\prime} \in U$ and $b_{0} \equiv_{n} a_{0,0}^{\prime}$. In either case, $a_{i, 0}^{\prime}$ together with $a_{\ell, 0}($ for $\ell \neq i)$ provide a matching partition of $\mathcal{A}$ into $n$-indecomposables.

Now we prove (2) $\Rightarrow$ (1) of Theorem 4.1 .

Lemma 4.4. For any Boolean algebra $\mathcal{A}$, if there is an ultrafilter $U$ such that $\mathcal{A} \equiv_{n} \mathcal{A}\lceil$ a for all $a \in U$, then $\mathcal{A}$ is n-indecomposable for $U$.

Proof. Let $U$ be an ultrafilter such that $\mathcal{A} \equiv_{n} \mathcal{A} \uparrow a$ for all $a \in U$. Let $a \in U$ be $n$-indecomposable for $U$ (by Lemma 3.12(10). Then $\mathcal{A} \equiv_{n} \mathcal{A}\left\lceil a\right.$. Since $a \leq 1_{\mathcal{A}}$, it follows that $t_{n}(\mathcal{A}) \leq_{n}^{\mathrm{w}} t_{n}(a)$.

We will show the following:

$(*)$ For any $V \neq U, t_{n-1}(V) \in \in^{\mathrm{w}} t_{n}(a)$.

Let $V \neq U$ and choose pairwise disjoint $(n-1)$-indecomposable elements $b_{0} \in U$ and $b_{1} \in V$; then set $b_{2}=1_{\mathcal{A}}-\left(b_{0} \dot{\vee} b_{1}\right)$. Since $\mathcal{A}\left\lceil a \leq_{n} \mathcal{A}\right.$ and $a$ is $n$ indecomposable for $U$, by Lemma 4.3 there is a partition of $a,\left(a_{0}, a_{1}, a_{2}\right)$, into $(n-$ $1)$-indecomposables for some ultrafilter such that $\left(b_{0}, b_{1}, b_{2}\right) \leq_{n-1}\left(a_{0}, a_{1}, a_{2}\right)$. By Theorem 3.17. we then have $t_{n-1}(U) \leq_{n-1} t_{n-1}\left(a_{0}\right)$ and $t_{n-1}(V) \leq_{n-1} t_{n-1}\left(a_{1}\right)$. First, suppose that $a_{1} \in U$. We have that $t_{n-1}\left(a_{0}\right) \in{ }^{\mathrm{w}} t_{n}(a)$ by condition (ii) of Definition 3.5 as $a_{0}$ is $(n-1)$-indecomposable for some ultrafilter $W \neq U$. Thus, 
$t_{n-1}(U) \in^{\mathrm{w}} t_{n}(a)$. Since $a_{1} \leq a$, by Lemma 3.12(2) $a_{1}$ is $n$-indecomposable for $U$, and so

$$
t_{n-1}(V) \leq_{n-1} t_{n-1}\left(a_{1}\right) \equiv_{n-1} t_{n-1}(U) \in{ }^{\mathrm{w}} t_{n}(a) .
$$

Now, suppose that $a_{1} \notin U$. Then it follows that $t_{n-1}(V) \leq_{n-1} t_{n-1}\left(a_{1}\right) \in{ }^{\mathrm{w}} t_{n-1}(a)$ by condition (ii) of Definition 3.5 as $a_{1}$ is $(n-1)$-indecomposable for some ultrafilter $W \neq U$. Thus, $(*)$ holds.

It follows from $(*)$ that $t_{n}(a) \leq_{n}^{\mathrm{w}} t_{n}(\mathcal{A})$, and since $t_{n}(\mathcal{A}) \leq_{n}^{\mathrm{w}} t_{n}(a)$, from Lemma 3.8 (C) it follows that $t_{n}(\mathcal{A}) \equiv_{n} t_{n}(a) \equiv_{n} t_{n}(U)$; thus condition (i) of Definition 3.5 holds. Condition (ii) of Definition 3.5 follows straightforwardly from $(*)$ and $t_{n}(\mathcal{A}) \equiv_{n} t_{n}(a)$.

Since (2) $\Longrightarrow$ (3) follows from the property (Uf.a) of ultrafilters, there only remains to show that (3) $\Longrightarrow$ (2) to complete Theorem 4.1. Let $\mathcal{A}$ satisfy the conditions in (3), so that for every partition $\left(a_{i}\right)_{i<k}$ of $\mathcal{A}$ there is some $i \leq k$ with $\mathcal{A} \equiv_{n} \mathcal{A}\left\lceil a_{i}\right.$. Say $a \in \mathcal{A}$ is strongly $n$-equivalent to $\mathcal{A}$ if $\mathcal{A}\left\lceil a \equiv_{n} \mathcal{A}\right.$ and for any partition $\left(c_{i}\right)_{i \leq k}$ of $a$ there is some $i$ with $\mathcal{A} \equiv_{n} \mathcal{A} \uparrow c_{i}$. Note that (3) says that $1_{\mathcal{A}}$ is strongly $n$-equivalent to $\mathcal{A}$. We will show that for every $a \in \mathcal{A}$ which is strongly $n$-equivalent to $\mathcal{A}$, there is an ultrafilter $U \ni a$ all of whose members are strongly $n$-equivalent to $\mathcal{A}$. This ultrafilter will also be sufficient for (2). The proof is by induction on $n$, where the basis case $n=0$ is trivial, since every nonzero element of $\mathcal{A}$ is strongly 0 -equivalent to $\mathcal{A}$. Assume that if $a$ is strongly $(n-1)$ equivalent to $\mathcal{A}$, then there is an ultrafilter $V \ni a$ all of whose members are strongly $(n-1)$-equivalent to $\mathcal{A}$.

Claim 1. If $a \in \mathcal{A}$ is strongly $n$-equivalent to $\mathcal{A}$, then for every partition $\left(a_{i}\right)_{i \leq k}$ of $a$ there is an $i$ with $a_{i}$ strongly $n$-equivalent to $\mathcal{A}$.

Proof. Suppose $a \in \mathcal{A}$ is strongly $n$-equivalent to $\mathcal{A}$ and $\left(a_{i}\right)_{i \leq k}$ is a counterexample to the claim. Then for each $i$, there is a partition $\left(b_{i, j}\right)_{j \leq m_{i}}$ of $a_{i}$ for which there is no $j \leq m_{i}$ with $\mathcal{A} \equiv_{n} \mathcal{A} \uparrow b_{i, j}$. Now, $\left(b_{i, j}\right)_{i \leq k, j \leq m_{i}}$ is a partition of a with no $i \leq k, j \leq m_{i}$ satisfying $\mathcal{A} \equiv_{n} \mathcal{A} \uparrow b_{i, j}$, contradicting our assumption about $a$.

Claim 2. If $a$ is strongly $n$-equivalent to $\mathcal{A}$ and $a \leq b$, then $\mathcal{A} \equiv_{n} \mathcal{A} \uparrow b$.

Proof. Since $a$ is strongly $n$-equivalent to $\mathcal{A}$, it is also strongly $(n-1)$-equivalent to $\mathcal{A}$, so by the induction hypothesis there is an ultrafilter $V \ni a$ all of whose members are strongly $(n-1)$-equivalent to $\mathcal{A}$. It follows that $a$ is $(n-1)$-indecomposable for $V$.

We first show that $\mathcal{A} \leq_{n} \mathcal{A} \uparrow b$. Let $\left(b_{i}\right)_{i \leq k}$ be a partition of $b$ and therefore $\left(a \wedge b_{i}\right)_{i \leq k}$ is a partition of $a$. Thus, for some $i \leq k, a \wedge b_{i} \in V$, and in particular, $\mathcal{A} \uparrow a \wedge b_{i} \equiv_{n-1} \mathcal{A} \uparrow a \equiv_{n-1} \mathcal{A}$. We may suppose $i=0$; then let $c_{j}=b_{j}$ and $c_{0}=1_{\mathcal{A}}-\left(b_{1} \vee \ldots \vee b_{k}\right)$. Thus, $\left(c_{i}\right)_{i \leq k}$ is a partition of $\mathcal{A}$ and $c_{i} \equiv_{n-1} b_{i}$ for $i>0$, and for $i=0$ we have $c_{0} \in V$ since $a \wedge b_{0} \in V$ and $V$ is an ultrafilter. Therefore, $\left(c_{i}\right)_{i \leq k}$ is a partition of $\mathcal{A}$ with $\left(c_{i}\right)_{i \leq k} \geq_{n-1}\left(b_{i}\right)_{i \leq k}$. Thus, $\mathcal{A} \leq_{n} \mathcal{A}\lceil b$.

We now show that $\mathcal{A} \uparrow b \leq_{n} \mathcal{A}$. Now let $\left(c_{i}\right)_{i \leq k}$ be a partition of $1_{\mathcal{A}}$. Since $\mathcal{A} \uparrow a \leq_{n} \mathcal{A}$, there is a partition $\left(a_{i}\right)_{i \leq k}$ of $a$ with $\mathcal{A} \uparrow c_{i} \leq_{n} \mathcal{A} \uparrow a_{i}$. We may also suppose that $a_{0} \in V$ since $a \in V$ and $V$ is an ultrafilter. Let $b_{i}=a_{i}$ for $i>0$ and $b_{0}=b-\left(a_{0} \vee \ldots \vee a_{k}\right)$ so that $\left(b_{i}\right)_{i \leq k}$ is a partition of $b$. We still have $c_{i} \leq_{n-1} b_{i}$ for $i>0$. However, $\mathcal{A} \uparrow a_{0} \equiv_{n-1} \mathcal{A} \uparrow b_{0}$ since $a_{0} \in V$ and $a_{0} \leq b_{0}$ implies $b_{0} \in V$. Thus, $\left(b_{i}\right)_{i \leq k}$ is a partition of $b$ with $\left(c_{i}\right)_{i \leq k} \leq_{n-1}\left(b_{i}\right)_{i \leq k}$ so that $\mathcal{A}\left\lceil b \leq_{n} \mathcal{A}\right.$. 
Claim 3. If $a$ is strongly $n$-equivalent to $\mathcal{A}$ and $b \geq a$, then $b$ is strongly $n$-equivalent to $\mathcal{A}$.

Proof. Let $a$ be strongly $n$-equivalent to $\mathcal{A}$ and $b \geq a$. Consider a partition $\left(c_{i}\right)_{i<\ell}$ of $b$. Then $\left(c_{i} \wedge a\right)_{i<\ell}$ is a partition of $a$, and hence some $c_{i} \wedge a$ is strongly $n$-equivalent to $\mathcal{A}$ by the first claim. However, then, by the second claim, $\mathcal{A}\left\lceil c_{i} \equiv_{n} \mathcal{A}\right.$. Since the partition $\left(c_{i}\right)_{i<\ell}$ of $b$ was arbitrary, it follows that $b$ is strongly $n$-equivalent to $\mathcal{A}$.

Let $a$ be strongly $n$-equivalent to $\mathcal{A}$ and suppose that for every ultrafilter $U \ni a$, there is an element $b_{U} \in U$ which is not strongly $n$-equivalent to $\mathcal{A}$. Since $\operatorname{Ult}(\mathcal{A})$ is compact, so is the clopen set $\mathcal{O}_{a}=\{U \in \operatorname{Ult}(\mathcal{A}): U \ni a\}$, and so there is a finite set $\left\{b_{U_{0}}, \ldots, b_{U_{\ell}}\right\}$ such that $b_{U_{0}} \vee \ldots \vee b_{U_{\ell}}=a$. (This argument is as given in the proof of Theorem 3.13.) Then there is a partition $\left(b_{i, j}\right)_{i \leq \ell, j \leq m_{i}}$ of $a$ with $b_{U_{i}}=\bigvee_{j \leq m_{i}} b_{i, j}$. Since none of the $b_{U_{i}}$ are strongly $n$-equivalent to $\mathcal{A}$, by the third claim none of the $b_{i, j}$ can be strongly $n$-equivalent to $\mathcal{A}$, contradicting the hypothesis that $a$ is strongly $n$-equivalent to $\mathcal{A}$. Therefore, there is an ultrafilter $U \ni a$ all of whose members are strongly $n$-equivalent to $\mathcal{A}$.

\section{REALIZABLE $n$-BF-INDECOMPOSABLE TYPES}

In this section we characterize the $\leq_{n}$-antichains of $\mathbf{A B F}_{n}$ which are realized in some $(n+1)$-indecomposable Boolean algebra.

Definition 5.1. We say that $\alpha \subseteq \mathbf{A B F}_{n}$ is realizable if there exists an $(n+1)$ indecomposable Boolean algebra $\mathcal{A}$ such that $t_{n+1}(\mathcal{A})=\alpha$. We use $\mathbf{B F}_{n}$ to denote the set of realizable antichains of $\mathbf{A B F}_{n-1}$.

Theorem 5.3 below gives a simple combinatorial property that characterizes the realizable $n$-bf-types. Moreover, for every realizable $n$-bf-type, the proof explicitly builds a (computable) Boolean algebra of that type.

One reason why an antichain $\alpha \subseteq \mathbf{A B F}_{n}$ might not be realizable is the following. Suppose $\delta \in \gamma \in \alpha$ and $t_{n+1}(\mathcal{A})=\alpha$. Then $\mathcal{A}$ has infinitely many ultrafilters of $n$-bf-type $\gamma$ (see the proof of Lemma 3.10). Each of these ultrafilters is surrounded by ultrafilters of $(n-1)$-bf-type $\delta$. Therefore, $\mathcal{A}$ has infinitely many ultrafilters of $(n-1)$-bf-type $\delta$. Thus, there has to be some $\beta \in \mathbf{B F}_{n}$ with $(\beta)_{n-1}=\delta$ such that $\mathcal{A}$ has infinitely many ultrafilters of $n$-bf-type $\beta$. Note that $\beta \in{ }^{\mathrm{w}} \alpha$ and $\gamma \leq_{n}^{\mathrm{w}} \beta$ by Lemma 3.10. Therefore, a necessary condition for $\alpha$ to be realizable is that for every $\delta \in \gamma \in \alpha$ there exists $\beta \in{ }^{\mathrm{w}} \alpha$ with $(\beta)_{n-1}=\delta$ and $\gamma \leq_{n}^{\mathrm{w}} \beta$. This is the gist of Theorem $[5.3$

We define another binary relation on $\mathbf{A B} \mathbf{F}_{n}$.

Definition 5.2. For $\gamma, \delta \in \mathbf{A B F}_{n}$, let $\gamma \triangleleft_{n} \delta$ if

$$
(\delta)_{n-1} \in{ }^{\mathrm{w}} \gamma \& \gamma \leq_{n}^{\mathrm{w}} \delta .
$$

The reason why this relation is interesting comes from Lemma 3.10. If $\mathcal{A}$ is $n$ indecomposable for $U$ and $V \in \operatorname{Ult}(\mathcal{A}), V \neq U$, then $t_{n}(\mathcal{A}) \triangleleft_{n} t_{n}(V)$. It is not hard to show that $\triangleleft_{n}$ is a transitive relation on $\mathbf{A} \mathbf{B} \mathbf{F}_{n}$. However, $\triangleleft_{n}$ is not an ordering relation, because for some $\gamma \in \mathbf{A} \mathbf{B} \mathbf{F}_{n}$ we have $\gamma \triangleleft_{n} \gamma$, and for other $\gamma \in \mathbf{A B F}_{n}$, we have $\gamma \not_{n} \gamma$. An example of this will be given later in Section 6. Example 6.3. 
Theorem 5.3. An antichain $\alpha \subseteq \mathbf{A B F}_{n}$ is realizable if and only if every $\gamma \in \alpha$ is realizable and

$$
\forall \delta \in \gamma \exists \beta \in \alpha\left(\gamma \leq_{n}^{w} \beta \&(\beta)_{n-1}=\delta\right) .
$$

Proof. For the "only if" part, suppose $\mathcal{A}$ is $(n+1)$-indecomposable for $U, t_{n+1}(\mathcal{A})=$ $\alpha$, and $\delta \in \gamma \in \alpha$. Then there are infinitely many ultrafilters $V \in \operatorname{Ult}(\mathcal{A})$ with $t_{n}(V)=\gamma$. For each of these $V$, let $a_{V} \in \mathcal{A}$ be $n$-indecomposable for $V$ and let $W_{V} \in \operatorname{Ult}\left(\mathcal{A}\left\lceil a_{V}\right)\right.$ be such that $t_{n-1}\left(W_{V}\right)=\delta$. Therefore, $\gamma=t_{n}\left(a_{V}\right) \triangleleft_{n} t_{n}\left(W_{V}\right)$ by Lemma 3.10. There is some $\beta \in \alpha$ with $t_{n}\left(W_{V}\right) \leq_{n} \beta$ for infinitely many $W_{V}$, and for any such $W_{V},(\beta)_{n-1}=t_{n-1}\left(W_{V}\right) \in \gamma$ and $\gamma \leq_{n}^{\mathrm{w}} t_{n}\left(W_{V}\right) \leq_{n}^{\mathrm{w}} \beta$. Therefore, for every $\delta \in \gamma \in \alpha$, there exists $\beta \in \alpha$ such that $(\beta)_{n-1}=\delta$ and $\gamma \leq_{n}^{\mathrm{w}} \beta$.

For the "if" part, suppose $\alpha$ satisfies the condition of the theorem; we will construct an $(n+1)$-indecomposable Boolean algebra $\mathcal{A}$ with $t_{n+1}(\mathcal{A})=\alpha$. The construction of $\mathcal{A}$ is essentially from [FZ80.

Note that for each $\gamma \in \alpha$, the witness $\beta$ in the condition of the theorem satisfies $\gamma \triangleleft_{n} \beta$. Moreover, the condition of the theorem implies that

$$
\gamma \equiv_{n}\left\{(\beta)_{n-1}: \beta \in \alpha, \gamma \triangleleft_{n} \beta\right\} .
$$

Let $\alpha^{\prime}=(\alpha)_{n}$ and $\alpha^{\infty}=\alpha \cup\left\{\alpha^{\prime}\right\}$. Then $\alpha^{\prime} \triangleleft_{n} \gamma$ for each $\gamma \in \alpha$, since the conditions of the theorem imply $\gamma \subseteq \alpha^{\prime}$. Therefore, $\alpha=\left\{\beta: \beta \in \alpha, \alpha^{\prime} \triangleleft_{n} \beta\right\}$ and $\alpha^{\prime} \equiv_{n}\left\{(\beta)_{n-1}: \beta \in \alpha, \alpha^{\prime} \triangleleft_{n} \beta\right\}$. We note that $\triangleleft_{n}$ is transitive and antisymmetric. However, there are some $\chi \in \mathbf{B F}_{n}$ for which $\chi \triangleleft_{n} \chi$ and others for which $\chi \not_{n} \chi$.

We will require two operations on Boolean algebras. The free product of $\omega$ copies of $\mathcal{A}$, denoted by $\bigoplus_{\omega} \mathcal{A}$, is the Boolean algebra whose domain consists of the infinite sequences $\left(a_{i}: i \in \omega\right)$ with $a_{i} \in \mathcal{A}$ and such that either $a_{i}=1_{\mathcal{A}}$ for cofinitely many $i \in \omega$ or $a_{i}=0_{\mathcal{A}}$ for cofinitely many $i \in \omega$. The Boolean algebra operations are defined componentwise. (See [Mon89, Chapter 4, Section 11] for an equivalent definition and some background.) The shuffle-product of a Boolean algebra $\mathcal{A}$, denoted by $\bigoplus_{\eta} \mathcal{A}$, is the Boolean algebra whose domain consists of sequences $\left(a_{\sigma}: \sigma \in 2^{<\omega}\right)$ with $a_{\sigma} \in \mathcal{A}$ and such that for some $j \in \omega$ and for each $\tau \in 2^{j}$, either $a_{\sigma}=1_{\mathcal{A}}$ for every $\sigma \supseteq \tau$ or $a_{\sigma}=0_{\mathcal{A}}$ for every $\sigma \supseteq \tau$. The Boolean algebra operations are defined componentwise.

For $\chi \in \alpha^{\infty}$, we define $\mathcal{A}_{\chi}^{\alpha}$ as follows. Let $P_{\chi}^{\alpha}=\left\{\zeta \in \alpha: \chi \triangleleft_{n} \zeta, \chi \neq \zeta\right\}$. The definition will be by recursion on the size of the set $P_{\chi}^{\alpha}$, since if $\zeta \in P_{\chi}^{\alpha}$, then $P_{\zeta}^{\alpha} \subsetneq P_{\chi}^{\alpha}$.

(1) If $P_{\chi}^{\alpha}=\emptyset$ and $\chi \nexists_{n} \chi$, then let $\mathcal{A}_{\chi}^{\alpha}$ be the two element Boolean algebra. If $P_{\chi}^{\alpha}=\emptyset$ and $\chi \triangleleft_{n} \chi$, then let $\mathcal{A}_{\chi}^{\alpha}$ be the atomless Boolean algebra.

(2) If $\chi \rtimes_{n} \chi$, then let

$$
\mathcal{A}_{\chi}^{\alpha}=\bigoplus_{\omega}\left(\bigoplus_{\zeta \in P_{\chi}^{\alpha}} \mathcal{A}_{\zeta}^{\alpha}\right)
$$

(3) If $\chi \triangleleft_{n} \chi$, then let

$$
\mathcal{A}_{\chi}^{\alpha}=\bigoplus_{\eta}\left(\bigoplus_{\zeta \in P_{\chi}^{\alpha}} \mathcal{A}_{\zeta}^{\alpha}\right)
$$

Finally, let $\mathcal{A}_{\alpha}=\mathcal{A}_{\alpha^{\prime}}^{\alpha}$.

There are two key facts about $\mathcal{A}_{\chi}^{\alpha}$. The first fact is that every relative algebra $\mathcal{A}_{\chi}^{\alpha}\left\lceil a\right.$ of $\mathcal{A}_{\chi}^{\alpha}$ is isomorphic to a finite sum of Boolean algebras isomorphic to either $\mathcal{A}_{\zeta}^{\alpha}$ for $\chi \triangleleft_{n} \zeta$ or to $\mathcal{A}_{\chi}^{\alpha}$. Also, in case (2) of the definition above, $\mathcal{A}_{\chi}^{\alpha}$ can appear at most once in the sum. Here is a sketch of a proof. The proof is by induction on $\chi$ as 
ordered by the relation $\triangleleft_{n}$. Let $\mathcal{A}=\bigoplus_{\zeta \in P_{\chi}^{\alpha}} \mathcal{A}_{\zeta}^{\alpha}$. By the induction hypothesis every relative algebra of $\mathcal{A}$ is isomorphic to a finite sum of Boolean algebras isomorphic to $\mathcal{A}_{\zeta}^{\alpha}$ for $\zeta \in P_{\chi}^{\alpha}$. Suppose first that $\mathcal{A}_{\chi}^{\alpha}$ was defined by case (2), i.e., that $\mathcal{A}_{\chi}^{\alpha}=$ $\bigoplus_{\omega} \mathcal{A}$. Consider $a=\left(a_{0}, a_{1}, \ldots\right) \in \mathcal{A}_{\chi}^{\alpha}$, where $a_{i} \in \mathcal{A}$. If for some $i_{0} \in \omega$, $\forall i \geq i_{0}\left(a_{i}=0_{\mathcal{A}}\right)$, then $\mathcal{A}_{\chi}^{\alpha}\left\lceil a\right.$ can be viewed as a relative algebra of $\bigoplus_{i=0}^{i_{0}} \mathcal{A}$, and hence by the inductive hypothesis, $\mathcal{A}_{\chi}^{\alpha} \uparrow a$ is isomorphic to a finite sum of Boolean algebras isomorphic to $\mathcal{A}_{\zeta}^{\alpha}$ for $\chi \triangleleft_{n} \zeta$. Otherwise, for some $i_{0}, \forall i \geq i_{0}\left(a_{i}=1_{\mathcal{A}}\right)$. In this case we can view $\mathcal{A}_{\chi}^{\alpha} \uparrow a$ as a relative algebra of $\bigoplus_{i=1}^{i_{0}} \mathcal{A}$ plus a copy of $\mathcal{A}_{\chi}^{\alpha}$. Hence, by inductive hypothesis, $\mathcal{A}_{\chi}^{\alpha}$ is isomorphic to a finite sum of Boolean algebras isomorphic to either $\mathcal{A}_{\zeta}^{\alpha}$ for $\chi \triangleleft_{n} \zeta$ or to $\mathcal{A}_{\chi}^{\alpha}$, and $\mathcal{A}_{\chi}^{\alpha}$ appears once in the sum. Suppose now that $\mathcal{A}_{\chi}^{\alpha}$ was defined by case (3), so $\mathcal{A}_{\chi}^{\alpha}=\bigoplus_{\eta} \mathcal{A}$. Consider $a=\left(a_{\sigma}: \sigma \in 2^{<\omega}\right) \in \mathcal{A}_{\chi}^{\alpha}$, where $a_{\sigma} \in \mathcal{A}$. Therefore, there exists $i_{0}$ such that for every $\tau \in 2^{i_{0}}$, either $a_{\sigma}=1_{\mathcal{A}}$ for every $\sigma \supseteq \tau$ or $a_{\sigma}=0_{\mathcal{A}}$ for every $\sigma \supseteq \tau$. For $\tau \in 2^{i_{0}}$, define $e^{\tau}=\left(e_{\sigma}^{\tau}: \sigma \in 2^{<\omega}\right) \in A_{\chi}^{\alpha}$ to be such that $e_{\sigma}^{\tau}=1_{\mathcal{A}}$ for $\sigma \supseteq \tau$, and $e_{\sigma}^{\tau}=0_{\mathcal{A}}$ for $\sigma \nsupseteq \tau$. Note that $\mathcal{A}_{\chi}^{\alpha} \uparrow e^{\tau} \cong \mathcal{A}_{\chi}^{\alpha}$. There exists a finite set $F \subseteq 2^{i_{0}}$ such that $a=b \dot{\vee} \bigvee_{\tau \in F} e^{\tau}$, where $b=\left(b_{\sigma}: \sigma \in 2^{<\omega}\right)$ and $b_{\sigma}=0$ for each $\sigma \in 2^{<\omega}$ with $|\sigma| \geq i_{0}$. By the inductive hypothesis, $\mathcal{A}_{\chi}^{\alpha} \uparrow b$ is isomorphic to a finite sum of Boolean algebras isomorphic to $\mathcal{A}_{\zeta}^{\alpha}$ for $\zeta \in P_{\chi}^{\alpha}$. Therefore, $\mathcal{A}_{\chi}^{\alpha}$ is isomorphic to a finite sum of Boolean algebras isomorphic to either $\mathcal{A}_{\zeta}^{\alpha}$ for $\zeta \in P_{\chi}^{\alpha}$ or $\mathcal{A}_{\chi}^{\alpha}$. This first fact implies that $\mathcal{A}_{\chi}^{\alpha}$ is $m$-indecomposable for every $m$. (Moreover, $\mathcal{A}$ is pseudo-indecomposable in the sense of [Pie89, §3] or [Hei92.)

The second fact is that for every $\zeta \in \alpha$, if $\chi \triangleleft_{n} \zeta$, then there are infinitely many disjoint relative algebras of $\mathcal{A}_{\chi}^{\alpha}$ isomorphic to $\mathcal{A}_{\zeta}^{\alpha}$.

It follows from these two facts that for every $m \leq n$,

$$
t_{m}\left(\mathcal{A}_{\chi}^{\alpha}\right) \equiv_{m}\left\{t_{m-1}\left(\mathcal{A}_{\zeta}^{\alpha}\right): \zeta \in \alpha, \chi \triangleleft_{n} \zeta\right\}
$$

As we noted earlier for each $\chi \in \alpha^{\infty}, \chi \equiv_{n}\left\{(\zeta)_{n-1}: \zeta \in \alpha, \chi \triangleleft_{n} \zeta\right\}$. Therefore, $(\chi)_{m} \equiv_{m}\left\{(\zeta)_{m-1}: \zeta \in \alpha, \chi \triangleleft_{n} \zeta\right\}$ for each $m \leq n$. It follows by induction on $m \leq n$ that $t_{m}\left(\mathcal{A}_{\chi}^{\alpha}\right)=(\chi)_{m}$ for every $\chi \in \alpha^{\infty}$. Since $\alpha=\left\{\zeta: \zeta \in \alpha, \alpha^{\prime} \triangleleft_{n} \zeta\right\}$, it follows that $\mathcal{A}_{\alpha^{\prime}}$ has $(n+1)$-bf-type $\alpha$, as required.

We remark that the construction of $\mathcal{A}_{\alpha}$ in the proof from the "if" direction is computable uniformly in $\alpha$.

Corollary 5.4. There is a uniformly computable procedure taking realizable $\alpha \in$ $\mathbf{B F}_{n}$ to an $n$-indecomposable Boolean algebra $\mathcal{A}_{\alpha}$ with $t_{n}\left(\mathcal{A}_{\alpha}\right)=\alpha$.

5.1. Interval algebras. It is easier to describe the examples from the construction in Theorem 5.3 using interval algebras. Let $\mathcal{L}$ be a linear order with a smallest element. We write $\operatorname{Int}(\mathcal{L})$, the interval algebra of $\mathcal{L}$, for the Boolean algebra generated from the sets $[x, y)$ where $x \in \mathcal{L}$ and $y \in \mathcal{L} \cup\{+\infty\}$. The elements of $\operatorname{Int}(\mathcal{L})$ are finite unions of half-open intervals $[x, y)$. Every countable Boolean algebra is isomorphic to an interval algebra generated from some countable linear order. See Mon89, Chapter 6, Section 15] for more information on interval algebras.

The operations we have been using on Boolean algebras correspond to operations on linear orderings. For example, for linear orderings $\mathcal{L}_{0}$ and $\mathcal{L}_{1}$,

$$
\operatorname{Int}\left(\mathcal{L}_{0}\right) \oplus \operatorname{Int}\left(\mathcal{L}_{1}\right)=\operatorname{Int}\left(\mathcal{L}_{0}+\mathcal{L}_{1}\right) .
$$


It is also not hard to show that for every linear ordering $\mathcal{L}$,

$$
\bigoplus_{\omega} \operatorname{Int}(\mathcal{L})=\operatorname{Int}(\mathcal{L} \cdot \omega)
$$

and

$$
\bigoplus_{\eta} \operatorname{Int}(\mathcal{L})=\operatorname{Int}((\mathcal{L}+1) \cdot \breve{\eta})
$$

where $\eta$ is the order type of the rational numbers and $\breve{\eta}=(1+\eta)$ is the order type of the rationals in the interval $[0,1)$.

Now, given $\chi \in \alpha^{\infty}$, we define a linear ordering $\mathcal{I}_{\chi}^{\alpha}$ as follows:

(1) If $P_{\chi}^{\alpha}=\emptyset$ and $\chi \nexists_{n} \chi$, then $\mathcal{I}_{\chi}^{\alpha}=1$, the linear ordering with one element. If $P_{\chi}^{\alpha}=\emptyset$ and $\chi \triangleleft_{n} \chi$, then let $\mathcal{I}_{\chi}^{\alpha}=\breve{\eta}$, the order type of the rationals in the interval $[0,1)$.

(2) If $\chi \nexists_{n} \chi$, then

$$
\mathcal{I}_{\chi}^{\alpha}=\left(\sum_{\zeta \in P_{\chi}^{\alpha}} \mathcal{I}_{\zeta}^{\alpha}\right) \cdot \omega
$$

(3) If $\chi \triangleleft_{n} \chi$, then

$$
\mathcal{I}_{\chi}^{\alpha}=\left(\sum_{\zeta \in P_{\chi}^{\alpha}} \mathcal{I}_{\zeta}^{\alpha}+1\right) \cdot \breve{\eta}
$$

Finally, let $\mathcal{I}_{\alpha}=\mathcal{I}_{\alpha^{\prime}}^{\alpha}$.

It is not hard to prove by induction on $\chi$ that $\mathcal{A}_{\chi}^{\alpha}=\operatorname{Int}\left(I_{\chi}^{\alpha}\right)$ and $\mathcal{A}_{\alpha}=\operatorname{Int}\left(\mathcal{I}_{\alpha}\right)$.

We will use this method to build examples of all bf-types through level five in Section 6 In some cases we can simplify the description of the linear ordering. The next lemma, stated without proof, presents a few simplifications we will make.

Lemma 5.5. (a) $\breve{\eta} \cdot \omega \cong \breve{\eta} \cdot \breve{\eta} \cong \breve{\eta}$.

$$
\text { (b) } \operatorname{Int}((L+\breve{\eta}) \cdot \omega) \cong \operatorname{Int}(L \cdot \omega+\eta) \text {. }
$$

\section{EXAMPLES OF INDECOMPOSABLES}

This section lists the realizable $n$-bf-types through level five and their relations. We have used a computer program to generate the $n$-bf-types through level six, where there are 1578 realizable 6 -bf-types. The computer program and the list of 6-bf-types is currently available online at the authors' web pages.

The naming convention used provides a name for each bf-type of the form $\ell_{i}$, where $\ell$ is a letter and $i$ is a number. The letter signifies the level, where $a$ is used for level 0 to $f$ for level 5 and $g$ for level 6 . The numbers distinguish types at a given level and have been assigned by our computer program. Two particular kinds of back-and-forth types are worth delineating.

Definition 6.1. We say $\alpha \in \mathbf{B F}_{n}$ is an isomorphism type if all Boolean algebras with bf-type $\alpha$ are isomorphic.

We say $\alpha \in \mathbf{B F}_{n}$ is an exclusive type if for any $n$-indecomposable Boolean algebra $\mathcal{A}$ with $t_{n}(\mathcal{A})=\alpha$ and any partition $\left(a_{0}, \ldots, a_{k}\right)$ into $(n-1)$-indecomposables, there is exactly one $i \leq k$ with $\mathcal{A}\left\lceil a_{i} \equiv_{n-1} \mathcal{A}\right.$.

Several names (each at a different level) may denote the same isomorphism type. For example, $b_{0}, c_{0}, d_{0}, e_{0}, f_{0}$ and $g_{0}$ all name the isomorphism type atom; the isomorphism type atomless is denoted by $c_{2}, d_{4}, e_{8}, f_{26}$ and $g_{1577}$. 
A necessary condition for an $n$-bf-type to be an isomorphism type is that there is exactly one descendant at every level $m>n . \quad\left(\beta \in \mathbf{B F}_{m}\right.$ is a descendant of $\alpha \in \mathbf{B F}_{n}$ if $m \geq n$ and $(\beta)_{n}=\alpha$.) We conjecture that a sufficient condition for an $n$-bf-type to be an isomorphism type is that it has exactly one descendant at level $n+1$. We corroborate this conjecture for $n \in\{1,2,3,4\}$ in the tables below.

On the other hand, a necessary and sufficient condition for a bf-type $\alpha \in \mathbf{B F}_{n}$ to be an exclusive type is that $(\alpha)_{n-1} \notin^{\mathrm{w}} \alpha$. The simplest example of an exclusive type is $b_{0}$, the isomorphism type of an atom. The other exclusive types through level 4 are also isomorphism types: $d_{1}$ (the type of the algebra $\operatorname{Int}(\omega)$, or 1-atom) and $e_{7}$ (the type of the algebra $\operatorname{Int}(\omega+\eta)$ ).

In the tables of the bf-types through level 5 that follow, we label the exclusive types by $\mathbf{X}$ and the isomorphism types by $\mathbf{I}$ in the column $\mathbf{I} / \mathbf{X}$. In the diagrams of the bf-types through level 5 that follow, we identify the exclusive and isomorphism types by using an enclosure as summarized in the following table:

$$
\text { isomorphism type nonisomorphism type }
$$

exclusive type

not exclusive

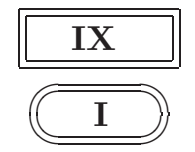

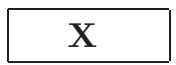

The interval algebras we provide in the tables for isomorphism types were obtained by linearizing the construction from Theorem 5.3 , as explained in Subsection 5.1 .

Up to level four, we describe the predicates $\mathrm{R}_{\alpha}$ (see the discussion in the introduction and in Definition 8.3) in terms of the Boolean algebra predicates used in KS00. The following are the predicates used in KS00:

- 1-predicates

- $\operatorname{atom}(x) \leftrightarrow \mathrm{R}_{b_{0}} \leftrightarrow x$ is an atom,

- 2-predicates

- atomless $(x) \leftrightarrow \mathrm{R}_{c_{2}} \leftrightarrow$ there are no atoms below $x$,

- infinite $(x) \leftrightarrow \mathrm{R}_{c_{1}} \leftrightarrow x$ is not a join of finitely many atoms,

- 3-predicates

- atomic $(x) \leftrightarrow \mathrm{R}_{d_{2}} \vee \neg \mathrm{R}_{c_{1}} \leftrightarrow x$ has no atomless elements below,

- 1-atom $(x) \leftrightarrow \mathrm{R}_{d_{1}} \leftrightarrow$ whenever $x=y \vee z$, exactly one of $y$ and $z$ is a finite join of atoms,

- $\operatorname{atominf}(x) \leftrightarrow \mathrm{R}_{d_{3}} \leftrightarrow$ there are infinitely many atoms below $x$,

- 4-predicates

$-\sim-\inf (x) \leftrightarrow \mathrm{R}_{c_{1}} \&\left(\neg \mathrm{R}_{d_{2}} \vee \mathrm{R}_{e_{2}}\right) \leftrightarrow$ there are infinitely many $\sim-$ nonequivalent elements below $x$, where $a \sim b$ if the symmetric difference $a \triangle b=(a-b) \vee(b-a)$ is finite.

- $\operatorname{Int}(\omega+\eta)(x) \leftrightarrow \mathrm{R}_{e_{7}} \leftrightarrow \operatorname{atominf}(x)$ and whenever $x=y \vee z$, there are only finitely many atoms below either $y$ or $z$.

- infatomicless $(x) \leftrightarrow \mathrm{R}_{e_{4}} \vee \mathrm{R}_{e_{8}} \vee \neg \mathrm{R}_{c_{1}} \leftrightarrow$ there is no infinite atomic element below $x$,

- 1-atomless $(x) \leftrightarrow \mathrm{R}_{e_{5}} \vee \mathrm{R}_{e_{3}} \vee \mathrm{R}_{e_{8}} \vee \neg \mathrm{R}_{c_{1}} \leftrightarrow$ there are no 1-atoms below $x$,

- $\operatorname{nomaxatomless}(x) \leftrightarrow \mathrm{R}_{e_{6}} \leftrightarrow x$ is not a join of atomless and atomic elements. 
We note that the $m$-predicates listed above are Boolean combinations of the predicates $\mathrm{R}_{\alpha}$ for $\alpha \in \mathbf{B F}_{n}$ for $n \leq m$ and that each of the predicates $\mathrm{R}_{\alpha}$ for $\alpha \in \mathbf{B F}_{m}$ is a Boolean combination of the $n$-predicates above for $n \leq m$.

In the diagrams below, an arrow $\ell_{i} \rightarrow \ell_{j}$ means that $\ell_{i} \geq_{n} \ell_{j}$ at the level $n$ being considered. The parent of an $n$-bf-type $\alpha$ is $(\alpha)_{n-1}$, and we say that $\alpha$ is a child of $(\alpha)_{n-1}$.

\section{0-indecomposable bf-type}

\begin{tabular}{|c|c|c|c|c|}
\hline Name & Members & Parent & $\mathrm{R}_{\alpha}$ & $\mathbf{I} / \mathbf{X}$ \\
\hline$a_{0}$ & & $a_{0}$ & nonzero element & \\
\hline
\end{tabular}

\section{1-indecomposable bf-types}

\begin{tabular}{|c|c|c|c|c|c|}
\hline Name & Members & Parent & $\mathrm{R}_{\alpha}$ & $\mathbf{I} / \mathbf{X}$ & Example \\
\hline$b_{0}$ & & $a_{0}$ & atom & $\mathbf{I X}$ & $\operatorname{Int}(2)$ \\
\hline$b_{1}$ & $a_{0}$ & $a_{0}$ & nonzero & & $\operatorname{Int}(\breve{\eta})$ \\
\hline
\end{tabular}

2-indecomposable bf-types

\begin{tabular}{|c|c|c|c|c|c|}
\hline Name & Members & Parent & $\mathrm{R}_{\alpha}$ & $\mathbf{I} \mathbf{X}$ & Example \\
\hline$c_{0}$ & & $b_{0}$ & atom & $\mathbf{I X}$ & $\operatorname{Int}(2)$ \\
\hline$c_{1}$ & $b_{0}$ & $b_{1}$ & infinite & & $\operatorname{Int}(\omega)$ \\
\hline$c_{2}$ & $b_{1}$ & $b_{1}$ & atomless & $\mathbf{I}$ & $\operatorname{Int}(\breve{\eta})$ \\
\hline
\end{tabular}

\section{bf-predicates for 1- and 2-indecomposable bf-types}
parent
$\underline{a_{0}}$
$\underline{b_{0}}$
$\underline{b_{1}}$
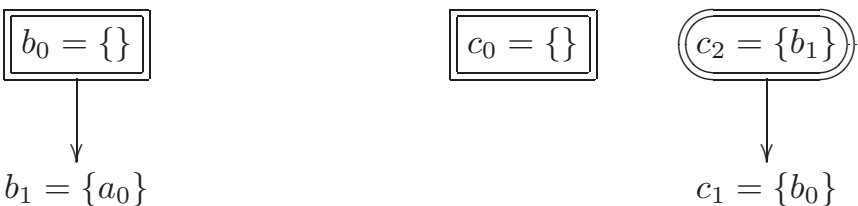

\section{3-indecomposable bf-types}

\begin{tabular}{|c|c|c|c|c|c|}
\hline Name & Members & Parent & $\mathrm{R}_{\alpha}$ & $\mathbf{I} \mathbf{X}$ & Example \\
\hline$d_{0}$ & & $c_{0}$ & atom & $\mathbf{I X}$ & $\operatorname{Int}(2)$ \\
\hline$d_{1}$ & $c_{0}$ & $c_{1}$ & 1-atom & $\mathbf{I X}$ & $\operatorname{Int}(\omega)$ \\
\hline$d_{2}$ & $c_{0}, c_{1}$ & $c_{1}$ & atomic \& infinite & & $\operatorname{Int}(2 \cdot \breve{\eta})$ \\
\hline$d_{3}$ & $c_{0}, c_{2}$ & $c_{1}$ & atominf & & $\operatorname{Int}(\omega+\eta)$ \\
\hline$d_{4}$ & $c_{2}$ & $c_{2}$ & atomless & $\mathbf{I}$ & $\operatorname{Int}(\breve{\eta})$ \\
\hline
\end{tabular}


ON THE $n$-BACK-AND-FORTH TYPES OF BOOLEAN ALGEBRAS

\section{bf-predicates for 3-indecomposable bf-types}

$$
\begin{aligned}
& \underline{\text { parent }} \quad \underline{c_{0}} \quad \underline{c_{1}} \quad \underline{c_{2}} \\
& \begin{array}{|ll}
d_{0}=\{\} & d_{1}=\left\{c_{0}\right\} \\
d_{4}=\left\{c_{2}\right\}
\end{array} \\
& d_{2}=\left\{c_{0}, c_{1}\right\} \\
& \downarrow \\
& d_{3}=\left\{c_{0}, c_{2}\right\}
\end{aligned}
$$

\section{4-indecomposable bf-types}

\begin{tabular}{|c|c|c|c|c|c|}
\hline Name & Members & Parent & $\mathrm{R}_{\alpha}$ & $\mathbf{I} / \mathbf{X}$ & Example \\
\hline$e_{0}$ & & $d_{0}$ & atom & IX & $\operatorname{Int}(2)$ \\
\hline$e_{1}$ & $d_{0}$ & $d_{1}$ & 1-atom & IX & $\operatorname{Int}(\omega)$ \\
\hline$e_{2}$ & $d_{0}, d_{1}$ & $d_{2}$ & atomic \& $\sim$-inf & & $\operatorname{Int}\left(\omega^{2}\right)$ \\
\hline$e_{3}$ & $d_{0}, d_{2}$ & $d_{2}$ & atomic \& $\sim$-inf \& 1-atomless & $\mathbf{I}$ & $\operatorname{Int}(2 \cdot \breve{\eta})$ \\
\hline$e_{4}$ & $d_{0}, d_{3}, d_{4}$ & $d_{3}$ & nomaxatomless \& infatomicless & & $\operatorname{Int}((2+\eta) \cdot \breve{\eta})$ \\
\hline$e_{5}$ & $d_{0}, d_{2}, d_{4}$ & $d_{3}$ & nomaxatomless \& 1-atomless & & $\operatorname{Int}(2 \cdot \breve{\eta}+\eta)$ \\
\hline$e_{6}$ & $d_{0}, d_{1}, d_{4}$ & $d_{3}$ & nomaxatomless & & $\operatorname{Int}\left(\omega^{2}+\eta\right)$ \\
\hline$e_{7}$ & $d_{0}, d_{4}$ & $d_{3}$ & Int $(\omega+\eta)$ & $\mathbf{I X}$ & $\operatorname{Int}(\omega+\eta)$ \\
\hline$e_{8}$ & $d_{4}$ & $d_{4}$ & atomless & $\mathbf{I}$ & $\operatorname{Int}(\breve{\eta})$ \\
\hline
\end{tabular}

In the next diagram we will omit the character ' $d$ ' and for example write $e_{5=\{0,2,4\}}$ instead of $e_{5}=\left\{d_{0}, d_{2}, d_{4}\right\}$.

\section{bf-predicates for 4-indecomposable bf-types}

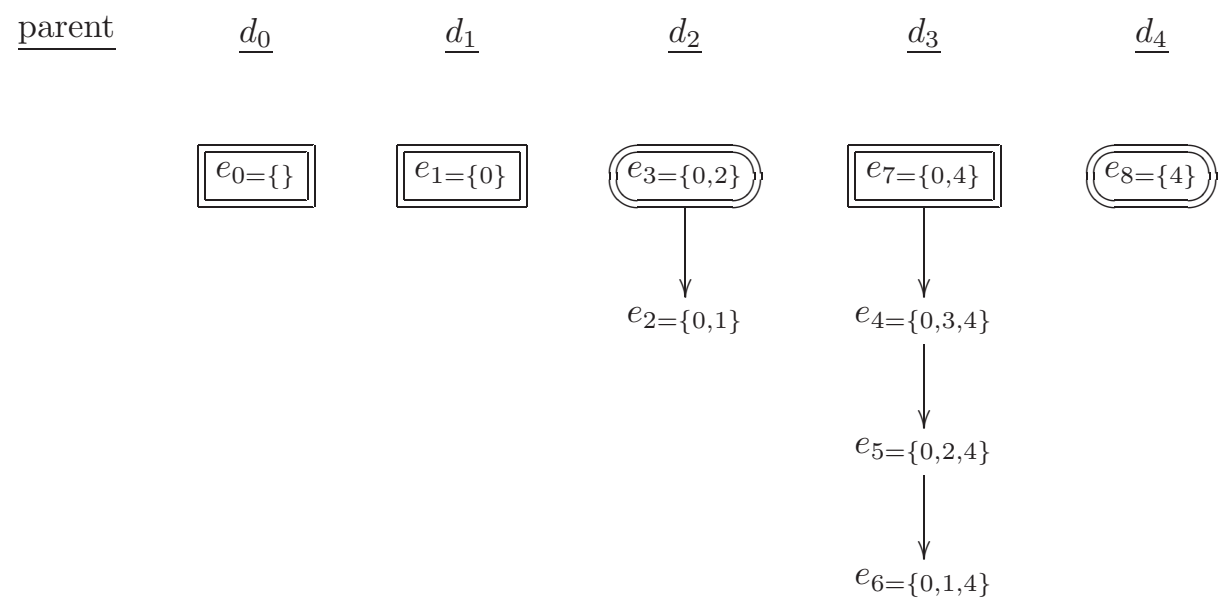




\section{5-indecomposable bf-types}

\begin{tabular}{|c|c|c|c|c|}
\hline Name & Members & Parent & $\mathbf{I} / \mathbf{X}$ & Example - Int $(\cdot)$ \\
\hline$f_{0}$ & & $e_{0}$ & IX & 1 \\
\hline$f_{1}$ & $e_{0}$ & $e_{1}$ & IX & $\omega$ \\
\hline$f_{2}$ & $e_{0}, e_{1}$ & $e_{2}$ & IX & $\omega^{2}$ \\
\hline$f_{3}$ & $e_{0}, e_{1}, e_{2}$ & $e_{2}$ & & $\omega^{2} \cdot \breve{\eta}$ \\
\hline$f_{4}$ & $e_{0}, e_{1}, e_{3}$ & $e_{2}$ & & $\omega^{2}+2 \cdot \eta$ \\
\hline$f_{5}$ & $e_{0}, e_{3}$ & $e_{3}$ & $\mathbf{I}$ & $2 \cdot \breve{\eta}$ \\
\hline$f_{6}$ & $e_{0}, e_{7}, e_{8}$ & $e_{4}$ & & $(\omega+\eta) \cdot \omega$ \\
\hline$f_{7}$ & $e_{0}, e_{1}, e_{7}, e_{8}$ & $e_{6}$ & & $(\omega+(\omega+\eta)) \cdot \omega$ \\
\hline$f_{8}$ & $e_{0}, e_{1}, e_{2}, e_{7}, e_{8}$ & $e_{6}$ & & $\left(\omega^{2} \cdot \breve{\eta}+(\omega+\eta)\right) \cdot \omega$ \\
\hline$f_{9}$ & $e_{0}, e_{1}, e_{3}, e_{7}, e_{8}$ & $e_{6}$ & & $(\omega+2 \cdot \breve{\eta}+(\omega+\eta)) \cdot \omega$ \\
\hline$f_{10}$ & $e_{0}, e_{3}, e_{7}, e_{8}$ & $e_{5}$ & & $(\omega+\eta) \cdot \omega+2 \cdot \eta$ \\
\hline$f_{11}$ & $e_{0}, e_{1}, e_{6}, e_{8}$ & $e_{6}$ & & $(\omega+\breve{\eta}) \cdot \breve{\eta}$ \\
\hline$f_{12}$ & $e_{0}, e_{1}, e_{2}, e_{6}, e_{8}$ & $e_{6}$ & & $\left(\omega^{2} \cdot \breve{\eta}+\breve{\eta}\right) \cdot \breve{\eta}$ \\
\hline$f_{13}$ & $e_{0}, e_{1}, e_{3}, e_{6}, e_{8}$ & $e_{6}$ & & $(\omega+2 \cdot \breve{\eta}+\breve{\eta}) \cdot \breve{\eta}$ \\
\hline$f_{14}$ & $e_{0}, e_{1}, e_{3}, e_{5}, e_{8}$ & $e_{6}$ & & $\omega^{2}+(2 \cdot \breve{\eta}+\breve{\eta}) \cdot \eta$ \\
\hline$f_{15}$ & $e_{0}, e_{3}, e_{5}, e_{8}$ & $e_{5}$ & & $(2 \cdot \breve{\eta}+\breve{\eta}) \cdot \breve{\eta}$ \\
\hline$f_{16}$ & $e_{0}, e_{4}, e_{8}$ & $e_{4}$ & $\mathbf{I}$ & $(2+\eta) \cdot \breve{\eta}$ \\
\hline$f_{17}$ & $e_{0}, e_{1}, e_{4}, e_{8}$ & $e_{6}$ & & $(\omega+2+\eta) \cdot \eta$ \\
\hline$f_{18}$ & $e_{0}, e_{1}, e_{2}, e_{4}, e_{8}$ & $e_{6}$ & & $\left(\omega^{2} \cdot \breve{\eta}+(2+\eta) \cdot \eta\right)$ \\
\hline$f_{19}$ & $e_{0}, e_{1}, e_{3}, e_{4}, e_{8}$ & $e_{6}$ & & $(\omega+2 \cdot \breve{\eta}) \cdot \omega+(2+\eta) \cdot \eta$ \\
\hline$f_{20}$ & $e_{0}, e_{3}, e_{4}, e_{8}$ & $e_{5}$ & & $2 \cdot \breve{\eta}+(2+\eta) \cdot \eta$ \\
\hline$f_{21}$ & $e_{0}, e_{3}, e_{8}$ & $e_{5}$ & IX & $2 \cdot \breve{\eta}+\eta$ \\
\hline$f_{22}$ & $e_{0}, e_{1}, e_{3}, e_{8}$ & $e_{6}$ & $\mathbf{X}$ & $(\omega+2 \cdot \breve{\eta}+\breve{\eta}) \cdot \omega$ \\
\hline$f_{23}$ & $e_{0}, e_{1}, e_{2}, e_{8}$ & $e_{6}$ & $\mathbf{X}$ & $\omega^{2} \cdot \breve{\eta}+\eta$ \\
\hline$f_{24}$ & $e_{0}, e_{1}, e_{8}$ & $e_{6}$ & IX & $\omega^{2}+\eta$ \\
\hline$f_{25}$ & $e_{0}, e_{8}$ & $e_{7}$ & IX & $\omega+\eta$ \\
\hline$f_{26}$ & $e_{8}$ & $e_{8}$ & $\mathbf{I}$ & $\breve{\eta}$ \\
\hline
\end{tabular}

We are not aware in the literature of a complete set of predicates identifying the twenty-seven 5-bf-types ( KS00] and Ala04 are through level four). In the following diagrams we will omit the character ' $e$ ' and write for example $f_{11=\{0,1,6,8\}}$ instead of $f_{11}=\left\{e_{0}, e_{1}, e_{6}, e_{8}\right\}$. 
ON THE $n$-BACK-AND-FORTH TYPES OF BOOLEAN ALGEBRAS

\section{bf-predicates for 5-indecomposable bf-types}
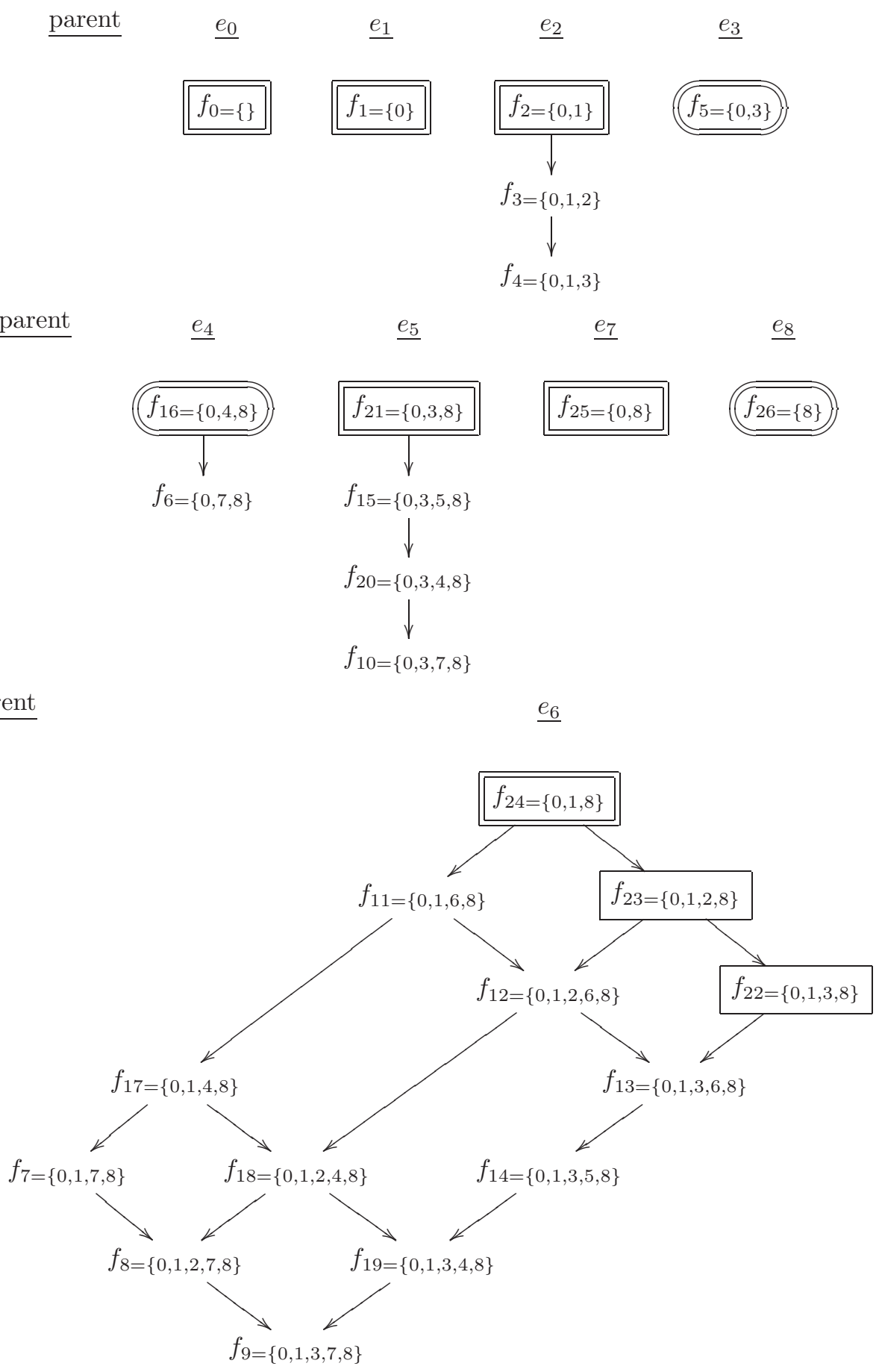

All bf-types through level four which are exclusive are also isomorphism types. However, not all exclusive types at level five are isomorphism types. Example 
6.2 below establishes that $f_{22}$ and $f_{23}$ are exclusive but not isomorphism types.

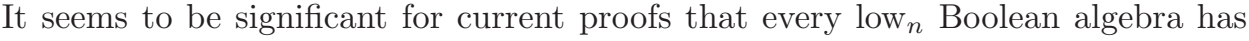
a computable copy (for $n \in\{1,2,3,4\}$ ), that the exclusive types at level $n$ are isomorphism types.

Example 6.2. The exclusive type $f_{23}$ has children $g_{1573}$ and $g_{1574}$ at level six (both are exclusive types):

(1) $g_{1573}$ has members $\left\{f_{0}, f_{1}, f_{3}, f_{26}\right\}$.

(2) $g_{1574}$ has members $\left\{f_{0}, f_{1}, f_{2}, f_{26}\right\}$.

Examples of Boolean algebras with 6 -bfthese types are $\operatorname{Int}\left(\left(\omega^{2} \cdot \breve{\eta}+\breve{\eta}\right) \cdot \omega\right)$ and $\operatorname{Int}\left(\left(\omega^{2}+\breve{\eta}\right) \cdot \omega\right)$, respectively.

Note that $g_{1574}<_{6} g_{1573}$.

The exclusive type $f_{22}$ has children $g_{1569}, g_{1570}, g_{1571}, g_{1572}$ at level six (all are exclusive types):

(1) $g_{1569}$ has members $\left\{f_{0}, f_{1}, f_{5}, f_{26}\right\}$.

(2) $g_{1570}$ has members $\left\{f_{0}, f_{1}, f_{2}, f_{5}, f_{26}\right\}$.

(3) $g_{1571}$ has members $\left\{f_{0}, f_{1}, f_{3}, f_{5}, f_{26}\right\}$.

(4) $g_{1572}$ has members $\left\{f_{0}, f_{1}, f_{4}, f_{5}, f_{26}\right\}$.

Examples of Boolean algebras with these 6 -bftypes are $\operatorname{Int}((\omega+2 \cdot \breve{\eta}+\breve{\eta}) \cdot \omega)$, $\operatorname{Int}\left(\left(\omega^{2}+2 \cdot \breve{\eta}+\breve{\eta}\right) \cdot \omega\right), \operatorname{Int}\left(\left(\omega^{2} \cdot \breve{\eta}+2 \cdot \breve{\eta}+\breve{\eta}\right) \cdot \omega\right)$ and $\operatorname{Int}\left(\left(\omega^{2}+2 \cdot \eta+\breve{\eta}\right) \cdot \omega\right)$, respectively.

Note that $g_{1570}<_{6} g_{1571}<_{6} g_{1572}<_{6} g_{1569}$.

Example 6.3. Here is an example of an antichain of $\mathbf{A B} \mathbf{F}_{2}$ which is not realizable. Consider $\left\{c_{1}\right\} \subseteq \mathbf{B F}_{2}$. One can easily check that it does not satisfy the property of Theorem 5.3. but here is a more intuitive reason. Suppose, toward a contradiction, that $\mathcal{A}$ is a Boolean algebra of 3-bftype $\left\{c_{1}\right\}$. Then, first $\mathcal{A}$ is infinite. Second, since $c_{0} \notin^{\mathrm{w}}\left\{c_{1}\right\}, \mathcal{A}$ cannot contain infinitely many atoms, and hence it must contain some atomless element. If it does contain an atomless element, it must contain infinitely many of them. However, this contradicts the fact that $c_{2} \notin^{\mathrm{w}}\left\{c_{1}\right\}$.

\section{General BACK-And-FOrth InVARIANtS}

In this section we define $n$-back-and-forth invariants for all Boolean algebras. Our approach is to consider how a Boolean algebra partitions into $n$-indecomposable elements. There are two complications here: first, there may be infinitely many ways a given Boolean algebra can be partitioned into $n$-indecomposable elements, and second, $\equiv_{n}$-equivalent Boolean algebras may not be partitionable into the same sequence of $n$-indecomposable elements.

We start with multisets of $\mathbf{B F}_{n}$ elements, and denote the collection of these by $\overline{\mathbf{I N V}}_{n}$. (A multiset is a collection where multiplicity is counted and order is ignored.)

Definition 7.1. Let $\left(\overline{\mathbf{I N V}}_{n},+, 0\right)$ be the free commutative monoid with generators $\mathbf{B F}_{n}$. That is, $+: \overline{\mathbf{I N V}}_{n} \times \overline{\mathbf{I N V}}_{n} \rightarrow \overline{\mathbf{I N V}}_{n}$ and $0 \in \overline{\mathbf{I N V}}_{n}$ satisfy for all $\rho, \sigma, \tau \in$ $\overline{\mathbf{I N V}}_{n}$ :

(a) Associativity: $(\rho+\sigma)+\tau=\rho+(\sigma+\tau)$.

(b) Commutativity: $\rho+\sigma=\sigma+\rho$.

(c) Identity: 0 is the unique element of $\overline{\mathbf{I N V}}_{n}$ satisfying $\rho+0=\rho=0+\rho$. 
A typical element of $\overline{\mathbf{I N V}}_{n}$ is of the form $\alpha_{0}+\ldots+\alpha_{k}$, where each $\alpha_{i} \in \mathbf{B F}_{n}$. (We will write this sum as $\sum_{i<k} \alpha_{i}$.) We will use $\pi, \rho, \sigma, \tau$ to denote elements of $\overline{\mathbf{I N V}}_{n}$ and $\alpha, \beta, \gamma, \delta$ to denote elements of $\mathbf{B F}_{n}$.

Definition 7.2. We define a map $\bar{T}_{n}$ from Boolean algebras to the powerset of $\overline{\mathbf{I N V}}_{n}$ by letting $\bar{T}_{n}(\mathcal{A})$ be the set of $\sum_{i \leq k} t_{n}\left(a_{i}\right) \in \overline{\mathbf{I N V}}_{n}$ where $\left(a_{i}\right)_{i \leq k}$ is a partition of $\mathcal{A}$ into $n$-indecomposables.

For $a \in \mathcal{A}$, we define $\bar{T}_{n}(a)$ to be $\bar{T}_{n}(\mathcal{A}\lceil a)$.

The problem with $\bar{T}_{n}(\mathcal{A})$ is that there are $\equiv_{n}$-equivalent Boolean algebras with different images under $\bar{T}_{n}$. We refine this definition by introducing a combinatorial relation $\leq_{n}$ on $\overline{\mathbf{I N V}}_{n}$, which will be based on how these elements can be partitioned into sequences of elements of $\overline{\mathbf{I N V}}_{n-1}$. The $n$-back-and-forth types are $\equiv_{n}$-equivalence classes of $\overline{\mathbf{I N V}}_{n}$, and the collection of these equivalence classes is denoted by $\mathbf{I N V}_{n}$. We then define a map from Boolean algebras to these equivalence classes and show that this provides an invariant for the $n$-back-and-forth types of Boolean algebras (Theorem 7.14). To get to the proof of Theorem 7.14, we need a sequence of lemmas about the relation $\leq_{n}$ on $\overline{\mathbf{I N V}}_{n}$.

We start the section by defining the notion of a partition for elements of $\mathbf{B F}_{n}$ and of $\overline{\mathbf{I N V}}_{n}$; we use these partitions to define the relation $\leq_{n}$ on $\overline{\mathbf{I N V}}_{n}$. Then, we prove that the addition operation on $\overline{\mathbf{I N V}}_{n}$ is compatible with the equivalence relation $\equiv_{n}$, allowing us to define addition on $\equiv_{n}$-equivalence classes for the quotient $\mathbf{I N V}_{n}$. Second, we show that the relation $\leq_{n}$ on $\overline{\mathbf{I N V}}_{n}$ is consistent with the relation $\leq_{n}$ on $\mathbf{B F}_{n}$ defined in Section 3 Third, we show that the relation $\leq_{n}$ on $\overline{\mathbf{I N V}}_{n}$ is consistent with the relation $\leq_{n}$ on the class of Boolean algebras defined in the introduction. Finally, we show that determining whether two elements of $\overline{\mathbf{I N V}}_{n}$ are in the same equivalence class is computable (Corollary 17.19). Again, we recommend that the reader skip the combinatorial proofs in a first read of the paper.

\subsection{Partitions of $n$-bf-types.}

Definition 7.3. Given $\alpha \in \mathbf{B F}_{n+1}$, a partition of $\alpha$ is a tuple $\left(\gamma_{i}\right)_{i \leq k}$ of $n$-bf-types such that $\gamma_{i_{0}} \equiv_{n}(\alpha)_{n}$ for some $i_{0} \leq k$ and $\gamma_{i} \in{ }^{\mathrm{w}} \alpha$ for each $i \neq i_{0}$.

Let $\left(\alpha_{i}\right)_{i \leq k}$ and $\left(\beta_{i}\right)_{i \leq k}$ be sequences of $n$-bf-types. We write $\left(\alpha_{i}\right)_{i \leq k} \leq_{n}\left(\beta_{i}\right)_{i \leq k}$ if $\alpha_{i} \leq_{n} \beta_{i}$ for all $i \leq k$.

The next lemma shows how these partitions relate to partitions of Boolean algebras.

Lemma 7.4. Let $\mathcal{A}$ be an $(n+1)$-indecomposable Boolean algebra for an ultrafilter U. Then:

(1) If $\left(a_{i}\right)_{i \leq k}$ is a partition of $\mathcal{A}$ into $n$-indecomposables, then $\left(t_{n}\left(a_{i}\right)\right)_{i \leq k}$ is a partition of $t_{n+1}(\mathcal{A})$.

(2) If $\left(\alpha_{i}\right)_{i \leq k}$ is a partition of $t_{n+1}(\mathcal{A})$, then there is a partition $\left(a_{i}\right)_{i \leq k}$ of $\mathcal{A}$ into $n$-indecomposables such that $\left(\alpha_{i}\right)_{i \leq k} \leq_{n}\left(t_{n}\left(a_{i}\right)\right)_{i \leq k}$.

Proof. (11) Let $\left(a_{i}\right)_{i \leq k}$ be a partition of $\mathcal{A}$ into $n$-indecomposables and $a_{0} \in U$. Since $a_{0} \in U, t_{n+1}\left(a_{0}\right) \equiv_{n+1} t_{n+1}(\mathcal{A})$, and so $t_{n}\left(a_{0}\right) \equiv_{n}\left(t_{n+1}(\mathcal{A})\right)_{n}$ by Lemma 3.9(2). It follows from Lemma 3.15)(2) that $t_{n}\left(a_{i}\right) \in{ }^{\mathrm{w}} t_{n+1}(\mathcal{A})$; thus $\left(t_{n}\left(a_{i}\right)\right)_{i \leq k}$ is a partition of $t_{n+1}(\mathcal{A})$. 
(2) We argue by induction on $k$. The case of $k=0$ trivially holds. Suppose $\left(\alpha_{i}\right)_{i \leq k+1}$ is a partition of $t_{n+1}(\mathcal{A})$ with $\alpha_{0}=t_{n}(\mathcal{A})$; then $\left(\alpha_{i}\right)_{i \leq k}$ is also a partition of $t_{n+1}(\mathcal{A})$. By the induction hypothesis there is a partition $\left(a_{i}\right)_{i \leq k}$ of $\mathcal{A}$ into $n$-indecomposables with $a_{0} \in U$ such that $\left(\alpha_{i}\right)_{i \leq k} \leq_{n}\left(t_{n}\left(a_{i}\right)\right)_{i \leq k}$. However, $t_{n+1}\left(a_{0}\right)=t_{n+1}(\mathcal{A})$ and $a_{0}$ is $(n+1)$-indecomposable, so by Lemma 3.15)(11) there is a partition $\left(a_{0}^{\prime}, a_{k+1}\right)$ of $a_{0}$ into $n$-indecomposables with $a_{0}^{\prime} \in U$ and $\alpha_{k+1} \leq_{n} t_{n}\left(a_{k+1}\right)$. Then $\left(a_{0}^{\prime}, a_{1}, \ldots, a_{k+1}\right)$ is the desired partition of $t_{n+1}(\mathcal{A})$.

Lemma 7.5. Let $\alpha, \beta \in \mathbf{B F}_{n}$. Then $\beta \leq_{n} \alpha$ if and only if for every partition $\left(\gamma_{i}\right)_{i \leq k}$ of $\alpha$ there is a partition $\left(\delta_{i}\right)_{i \leq k}$ of $\beta$ such that $\left(\gamma_{i}\right)_{i \leq k} \leq_{n-1}\left(\delta_{i}\right)_{i \leq k}$.

Proof. $(\Rightarrow)$. Suppose $\beta \leq_{n} \alpha$. Then $(\beta)_{n-1} \equiv_{n-1}(\alpha)_{n-1}$ and $\beta \leq_{n}^{\mathrm{w}} \alpha$. Let $\left(\gamma_{i}\right)_{i \leq k}$ be a partition of $\alpha$ with $\gamma_{0}=(\alpha)_{n-1}$. Define a partition $\left(\delta_{i}\right)_{i \leq k}$ of $\beta$ as follows: let $\delta_{0}$ be $(\beta)_{n-1}$ and, for each $1 \leq i \leq k$, let $\delta_{i} \in \beta$ satisfy $\gamma_{i} \leq_{n-1} \delta_{i}$ (using $\beta \leq_{n}^{\mathrm{w}} \alpha$ ). Then $\left(\gamma_{i}\right)_{i \leq k} \leq_{n-1}\left(\delta_{i}\right)_{i \leq k}$.

$(\Leftarrow)$. By Lemma 3.8(d) , we need only show $\beta \leq_{n}^{\mathrm{w}} \alpha$ and that $(\alpha)_{n-1} \leq_{n-1}$ $(\beta)_{n-1}$. For $\beta \leq_{n}^{\mathrm{w}} \alpha$, fix any $\gamma \in \alpha$. It is suffices to show that there is a $\delta \in \beta$ with $\gamma \leq_{n-1} \delta$. Consider the partition $\left((\alpha)_{n-1}, \gamma, \gamma\right)$ of $\alpha$. Then by hypothesis there is a partition $\left(\delta_{0}, \delta_{1}, \delta_{2}\right)$ of $\beta$ where $\gamma \leq_{n-1} \delta_{i}$ for $i \in\{1,2\}$; furthermore, $\delta_{i} \in{ }^{\mathrm{w}} \beta$ for some $i \in\{1,2\}$. Thus, there is a $\delta \in \beta$ with $\gamma \leq_{n-1} \delta_{i} \leq_{n-1} \delta$.

To show $(\alpha)_{n-1} \leq_{n-1}(\beta)_{n-1}$ consider the one element partition of $\alpha$, namely $\left((\alpha)_{n-1}\right)$. Since $\left((\beta)_{n-1}\right)$ is the only one element partition of $\beta$, by hypothesis, we have that $(\alpha)_{n-1} \leq_{n-1}(\beta)_{n-1}$.

7.2. The $n$-back-and-forth relation on $\overline{\mathbf{I N V}}_{n}$. We now define a relation $\leq_{n}$ on $\overline{\mathbf{I N V}}_{n}$. We will show later in Lemma 7.11 that this definition is an extension of the relation $\leq_{n}$ on $\mathbf{B F}_{n}$. Before that, we will treat these two relations as different.

Definition 7.6. A decomposition of $\sum_{i \leq s} \alpha_{i} \in \overline{\mathbf{I N V}}_{n}$ is an element $\sum_{j \leq t} \gamma_{j} \in$ $\overline{\mathbf{I N V}}_{n-1}$ such that there is a partition $X_{0}, \ldots, X_{s}$ of the set $\{0, \ldots, t\}$, where for each $i$ the sequence $\left(\gamma_{j}\right)_{j \in X_{i}}$ is a partition of $\alpha_{i}$. A partition of $\sigma=\sum_{i \leq s} \alpha_{i} \in \overline{\mathbf{I N V}}_{n}$ is a sequence $\left(\sigma_{k}\right)_{k \leq r}$ of elements of $\overline{\mathbf{I N V}}_{n-1}$ such that there is a decomposition $\sum_{j \leq t} \beta_{j}$ of $\sigma$ and a partition $Y_{0}, \ldots, Y_{r}$ of the set $\{0, \ldots, t\}$, where $\sigma_{k}=\sum_{j \in Y_{k}} \beta_{j}$.

Note that the elements $\gamma_{j}$ of a decomposition as above are in $\mathbf{B} \mathbf{F}_{n-1}$, while the elements $\sigma_{k}$ of a partition as above are in $\overline{\mathbf{I N V}}_{n-1}$. Also, note that the word partition is being used with four different meanings: there are partitions of a set, partitions of an element of a Boolean algebra, partitions of an element of $\mathbf{B F}_{n}$, and partitions of an element of $\overline{\mathbf{I N V}}_{n}$. In the figure below we have $\sum_{i \leq s, j \leq m_{i}} \beta_{i, j}$ is a decomposition of $\sigma=\sum_{i<s} \alpha_{i} \in \overline{\mathbf{I N V}}_{n}$, as for each $i \leq s,\left(\beta_{i, j}\right)_{j \leq m_{i}}$ is a partition of $\alpha_{i} \in \mathbf{B F}_{n}$. We also have that $\left(\sigma_{k}\right)_{k \leq r}$ is a partition of $\sigma$, where $\sigma_{k}=\sum_{i \leq s} \beta_{i, k} \in \overline{\mathbf{I N V}}_{n-1}$.

\begin{tabular}{cccccc|cc} 
& $\beta_{1,1}$ & $\beta_{1,2}$ & $\ldots$ & $\ldots$ & $\beta_{1, m_{1}}$ & $\alpha_{1}$ & \\
& $\beta_{2,1}$ & $\beta_{2,2}$ & $\ldots$ & $\ldots$ & $\beta_{2, m_{2}}$ & $\alpha_{2}$ & \\
$\mathbf{B F}_{n-1} \ni$ & $\vdots$ & $\vdots$ & $\ddots$ & $\ddots$ & $\vdots$ & $\vdots$ & $\in \mathbf{B F}_{n}$ \\
$\overline{\mathbf{I N V}}_{n-1} \ni$ & $\beta_{s, 1}$ & $\beta_{s, 2}$ & $\ldots$ & $\ldots$ & $\beta_{s, m_{s}}$ & $\alpha_{s}$ & \\
\cline { 2 - 6 } & $\sigma_{1}$ & $\sigma_{2}$ & $\ldots$ & $\ldots$ & $\sigma_{r}$ & $\sigma$ & $\in \overline{\mathbf{I N V}}_{n}$
\end{tabular}


Definition 7.7. We define a relation $\leq_{n}$ on $\overline{\mathbf{I N V}}_{n}$ by induction on $n$. For $n=0$ and $\sigma, \tau \in \overline{\mathbf{I N V}}_{0}$, we let $\sigma \leq_{0} \tau$ if $\sigma=0$ or $\tau \neq 0$. For $\sigma, \tau \in \overline{\mathbf{I N V}}_{n}$ we let $\sigma \leq_{n} \tau$ if for every partition $\left(\rho_{i}\right)_{i \leq k}$ of $\tau$ there is a partition $\left(\pi_{i}\right)_{i \leq k}$ of $\sigma$ such that $\rho_{i} \leq_{n-1} \pi_{i}$ for each $i \leq k$. We write $\left(\rho_{i}\right)_{i \leq k} \leq_{n-1}\left(\pi_{i}\right)_{i \leq k}$ when $\rho_{i} \leq_{n-1} \pi_{i}$ for each $i \leq k$.

It follows by induction on $n$ that $\leq_{n}$ is a transitive relation on $\overline{\mathbf{I N V}}_{n}$, where $\leq_{0}$ is clearly transitive. Let $\mathbf{I N V} \mathbf{V}_{n}$ be the quotient of $\overline{\mathbf{I N V}}_{n}$ over the equivalence relation $\equiv_{n}$ and denote its elements by $[\sigma]$, where $\sigma \in \overline{\mathbf{I N V}}_{n}$.

The next lemma shows that the operation + can be extended to $\mathbf{I N V}_{n}$ as a coset operation.

Lemma 7.8. Let $\sigma_{i}, \tau_{i} \in \overline{\boldsymbol{I N V}}_{n}$ (for $i \leq m$ ) be such that $\left(\sigma_{i}\right)_{i \leq m} \leq_{n}\left(\tau_{i}\right)_{i \leq m}$. Then $\sum_{i \leq m} \sigma_{i} \leq_{n} \sum_{i \leq m} \tau_{i}$.

Proof. The proof is by induction on $n$. Suppose $\left(\sigma_{i}\right)_{i \leq m} \leq_{n}\left(\tau_{i}\right)_{i \leq m}$ for sequences of elements from $\overline{\mathbf{I N V}}_{n}$. Let $\tau_{i}=\sum_{j \leq t_{i}} \beta_{i, j}$ and $\sigma_{i}=\sum_{j \leq s_{i}} \alpha_{i, j}$. Fix any partition $\left(\rho_{j}\right)_{j \leq \ell}$ of $\sum_{i \leq k} \tau_{i}$, and let $\sum_{k \leq t} \delta_{k}$ be a decomposition of $\sum_{i \leq k} \tau_{i}$ for which there is a partition $\left\{X_{i, j}\right\}$ of $\{0, \ldots, t\}$ where $\left(\delta_{k}\right)_{k \in X_{i, j}}$ is a partition of $\beta_{i, j}$, and a partition $\left\{Y_{j}\right\}$ of $\{0, \ldots, t\}$ where $\rho_{j}=\sum_{k \in Y_{j}} \delta_{k}$. Let $X_{i}=\bigcup_{j \leq t_{i}} X_{i, j}$ so that $\sum_{k \in X_{i}} \delta_{k}$ is a decomposition of $\tau_{i}$. Since $\sigma_{i} \leq_{n} \tau_{i}$ there is a partition $\left(\gamma_{k}\right)_{k \in X_{i}}$ of $\sigma_{i}$ where $\left(\delta_{k}\right)_{k \in X_{i}} \leq_{n-1}\left(\gamma_{k}\right)_{k \in X_{i}}$ (for all $i \leq m$ ). Let $\pi_{j}=\sum_{k \in Y_{j}} \gamma_{k}$ so that $\left(\rho_{j}\right)_{j \leq m} \leq_{n-1}\left(\pi_{j}\right)_{j \leq m}$ by the inductive hypothesis. Since the partition $\left(\rho_{j}\right)_{j \leq \ell}$ of $\sum_{i \leq k} \tau_{i}$ was arbitrary, it follows that $\sum_{i \leq m} \sigma_{i} \leq_{n} \sum_{i \leq m} \tau_{i}$.

Corollary 7.9. Let $\sigma, \tau \in \overline{\boldsymbol{I N}}_{n}$. Then $\sigma \leq_{n} \tau$ if and only if for every decomposition $\sum_{j \leq t} \gamma_{j}$ of $\tau$, there is a partition $\left(\sigma_{j}\right)_{j \leq t}$ of elements of $\overline{\boldsymbol{I N}}_{n-1}$ of $\sigma$ such that $\left(\gamma_{j}\right)_{j \leq t} \leq_{n-1}\left(\sigma_{j}\right)_{j \leq t}$.

It also follows from the preceding lemma that:

Theorem 7.10. ( IN $\left.\boldsymbol{V}_{n},+, 0, \leq_{n}\right)$ is a commutative ordered monoid.

7.3. Consistency between $\overline{\mathbf{I N V}}_{n}$ and $\mathbf{B} \mathbf{F}_{n}$. Next, we show that $\left(\mathbf{B F}{ }_{n}, \leq_{n}\right)$ is a subordering of $\left(\mathbf{I N V} \mathbf{V}_{n}, \leq_{n}\right)$. To avoid confusion in this subsection we will use $\leq_{n}^{I}$ to denote the relation $\leq_{n}$ on $\overline{\mathbf{I N V}}_{n}$ defined in Definition 7.7 and we will use $\leq_{n}^{B}$ to denote the relation $\leq_{n}$ on $\mathbf{B} \mathbf{F}_{n}$ defined in Definition 3.6.

Lemma 7.11. Let $\alpha, \beta \in \mathbf{B F}_{n}$. Then $\alpha \leq_{n}^{I} \beta$ as elements in $\overline{\mathbf{I N V}}_{n}$ if and only if $\alpha \leq_{n}^{B} \beta$ as elements of $\mathbf{B F}_{n}$.

This lemma follows from the case $k=0$ in the equivalence between (11) and (2) of the following lemma. Recall from Definition 5.2 that when $\alpha, \beta \in \mathbf{B F}_{n}$, we defined $\alpha \triangleleft_{n} \beta$ as the conjunction of $\alpha \leq_{n}^{\mathrm{w}} \beta$ and $(\beta)_{n-1} \in{ }^{\mathrm{w}} \alpha$.

Lemma 7.12. Let $\alpha, \beta_{0}, \ldots, \beta_{k} \in \mathbf{B F}_{n}$. The following are equivalent:

(1) $\alpha \leq_{n}^{I} \sum_{i \leq k} \beta_{i}$.

(2) For some $i_{0} \leq k$ we have $\alpha \leq_{n}^{B} \beta_{i_{0}}$, and for every other $i \neq i_{0}$ we have $\alpha \triangleleft_{n} \beta_{i}$.

Proof. The proof of the equivalence is by induction on $n$. We assume the equivalence holds for $n-1$, and note the following consequence of this assumption and (2):

(*) For any $\delta, \gamma_{0}, \ldots, \gamma_{m} \in \mathbf{B F}_{n-1}$, if $\delta \leq_{n-1}^{I} \sum_{j \leq m} \gamma_{j}$, then $\delta \leq_{n-1}^{\mathrm{w}} \gamma_{j}$ for each $j$. 
We prove that (11) implies (2). Suppose $\alpha \leq_{n}^{I} \sum_{i \leq k} \beta_{i}$. We first show that $\alpha \leq_{n}^{\mathrm{w}} \beta_{i}$ for each $i$. Let $\delta \in \beta_{i}$ and consider the decomposition of $\sum_{i \leq k} \beta_{i}$ of length $k+3$ given by $\sum_{i \leq k}\left(\beta_{i}\right)_{n-1}+\delta+\delta$. There is a partition $\left(\sigma_{j}\right)_{j \leq k+2}$ of $\alpha \in \overline{\mathbf{I N V}}_{n}$ such that $\sigma_{k+1} \geq_{n-1}^{I} \delta$ and $\sigma_{k+2} \geq_{n-1}^{I} \delta$. Each $\sigma_{j}$ is of the form $\sum_{i \leq m_{j}} \gamma_{j, i}$, and so by the induction hypothesis, for each $j \in\{k+1, k+2\}$ there exists $i_{j}$ such that $\delta \leq_{n-1}^{B} \gamma_{j, i_{j}}$. For at least one $j \in\{k+1, k+2\}$ we have that $\gamma_{j, i_{j}} \in{ }^{\mathrm{w}} \alpha$, and thus $\delta \in \in^{\mathrm{w}} \alpha$ as required.

To complete the argument, consider the decomposition of $\sum_{i \leq k} \beta_{i}$ given by $\sum_{i \leq k}\left(\beta_{i}\right)_{n-1}$. From (11), there is a partition $\left(\sigma_{i}\right)_{i \leq k}$ of $\alpha \in \overline{\mathbf{I N V}}_{n}$ such that $\left(\sigma_{i}\right)_{i \leq k} \geq_{n-1}^{I}\left(\left(\beta_{i}\right)_{n-1}\right)_{i \leq k}$. Write $\sigma_{i}$ as $\sum_{j \leq m_{i}} \gamma_{i, j}$, and without loss of generality suppose that $\gamma_{0,0}=(\alpha)_{n-1}$. By the induction hypothesis, for each $i$ there exists $j_{i}$ such that $\left(\beta_{i}\right)_{n-1} \leq_{n-1}^{B} \gamma_{i, j_{i}}$. Thus, when $i>0$ we have $\left(\beta_{i}\right)_{n-1} \leq_{n-1}^{B}$ $\gamma_{i, j_{i}} \in{ }^{\mathrm{w}} \alpha$, which together with $\alpha \leq_{n}^{\mathrm{w}} \beta_{i}$ implies $\alpha \triangleleft_{n} \beta_{i}$. When $i=0$, since $\left(\beta_{0}\right)_{n-1} \leq_{n-1}^{I} \sigma_{0}=\sum_{j \leq m_{0}} \gamma_{0, j}$, it follows from the induction hypothesis and $(*)$ that $\left(\beta_{0}\right)_{n-1} \leq_{n-1}^{\mathrm{w}} \gamma_{0,0}=(\alpha)_{n-1}$. Since both $\alpha \leq_{n}^{\mathrm{w}} \beta_{0}$ and $\left(\beta_{0}\right)_{n-1} \leq_{n-1}^{\mathrm{w}}(\alpha)_{n-1}$, it follows that $\alpha \leq_{n}^{B} \beta_{i_{0}}$ by Lemma 3.8.(d).

We prove that (2) implies (1). Suppose (2) holds and $i_{0}=0$, so $\alpha \leq_{n}^{B} \beta_{0}$. Let $\sum_{i<k, j \leq m_{i}} \gamma_{i, j} \in \overline{\mathbf{I N V}}_{n-1}$ be a decomposition of $\sum_{i \leq k} \beta_{i}$ where, for each $i,\left(\gamma_{i, j}\right)_{j \leq m_{i}}$ is a partition of $\beta_{i}$ and $\gamma_{i, 0}=\left(\beta_{i}\right)_{n-1}$. We now build a partition $\left(\delta_{i, j}\right)_{i \leq k, j \leq m_{i}} \subseteq \mathbf{B F}_{n-1}$ of $\alpha$ such that $\left(\gamma_{i, j}\right)_{i \leq k, j \leq m_{i}} \leq_{n-1}^{I}\left(\delta_{i, j}\right)_{i \leq k, j \leq m_{i}}$. By the inductive hypothesis of Lemma 7.11, it is enough to have $\left(\gamma_{i, j}\right)_{i \leq k, j \leq m_{i}} \leq_{n-1}^{B}$ $\left(\delta_{i, j}\right)_{i \leq k, j \leq m_{i}}$. Let $\delta_{0,0}=(\alpha)_{n-1}=\left(\beta_{0}\right)_{n-1}=\gamma_{0,0}$. For $i>0$, let $\delta_{i, 0}=\gamma_{i, 0}=$ $\left(\beta_{i}\right)_{n-1} \in{ }^{\mathrm{w}} \alpha$. For $j>0$, we have $\gamma_{i, j} \in{ }^{\mathrm{w}} \beta_{i} \geq_{n}^{\mathrm{w}} \alpha$, so there exists $\delta_{i, j} \in \alpha$ such that $\gamma_{i, j} \leq_{n-1}^{B} \delta_{i, j}$.

7.4. $n$-bf-invariants for Boolean algebras. Our goal is to assign an element of $\mathbf{I N V}_{n}$ to each Boolean algebra and to show that this element is an invariant for the relation $\leq_{n}$. We will define a map $T_{n}$ from Boolean algebras to $\mathbf{I N V _ { n }}$ such that for any Boolean algebras $\mathcal{A}$ and $\mathcal{B}$

$$
\mathcal{A} \leq_{n} \mathcal{B} \Longleftrightarrow T_{n}(\mathcal{A}) \leq_{n} T_{n}(\mathcal{B}) .
$$

We must be sure that it is possible to define such a map, $T_{n}$, on Boolean algebras. To this end it will be shown that if $\mathcal{A} \leq_{n} \mathcal{B}$ and $\left(a_{i}\right)_{i \leq k}$ and $\left(b_{j}\right)_{j \leq l}$ are partitions of $\mathcal{A}$ and $\mathcal{B}$ into $n$-indecomposables, then $\sum_{i \leq k} t_{n}\left(a_{i}\right) \leq_{n} \sum_{j \leq l} t_{n}\left(b_{j}\right)$ as members of $\overline{\mathbf{I N V}}_{n}$. In particular, it will follow that if $\left(a_{i}\right)_{i \leq k}$ and $\left(b_{j}\right)_{j \leq l}$ are different partitions of $\mathcal{A}$ into $n$-indecomposables, then $\sum_{i \leq k} t_{n}\left(a_{i}\right) \equiv_{n} \sum_{j \leq l} t_{n}\left(b_{j}\right)$.

Lemma 7.13. Let $\mathcal{A}$ be a Boolean algebra and $\sigma \in \bar{T}_{n}(\mathcal{A})$. Then:

(1) For every partition $\left(a_{i}\right)_{i \leq k}$ of $\mathcal{A}$, there is a partition $\left(\sigma_{i}\right)_{i \leq k}$ of $\sigma$ with $\sigma_{i} \in \bar{T}_{n-1}\left(a_{i}\right)$.

(2) For every decomposition $\sum_{j \leq t} \alpha_{j}$ of $\sigma$, there is a partition $\left(c_{j}\right)_{j \leq t}$ of $\mathcal{A}$ into $(n-1)$-indecomposables such that $\left(\alpha_{j}\right)_{j \leq t} \leq_{n-1}\left(t_{n-1}\left(c_{j}\right)\right)_{j \leq t}$.

(3) For every partition $\left(\sigma_{i}\right)_{i \leq k}$ of $\sigma$, there is a partition $\left(a_{i}\right)_{i \leq k}$ of $\mathcal{A}$ and $\tau_{i} \in$ $\bar{T}_{n-1}\left(a_{i}\right)$ (for each $i \leq k$ ) such that $\left(\sigma_{i}\right)_{i \leq k} \leq_{n-1}\left(\tau_{i}\right)_{i \leq k}$.

Proof. Let $\sigma \in \bar{T}_{n}(\mathcal{A})$ and $\left(b_{i}\right)_{i \leq s}$ be a partition of $\mathcal{A}$ into $n$-indecomposables such that $\sigma=\sum_{i \leq s} t_{n}\left(b_{i}\right)$. 
(11) Suppose $\left(a_{i}\right)_{i \leq k}$ is a partition of $\mathcal{A}$ and let $\left(c_{j}\right)_{j \leq t}$ be a common refinement of $\left(a_{i}\right)_{i \leq k}$ and $\left(b_{i}\right)_{i \leq s}$ into $(n-1)$-indecomposables. Thus, there is a partition $X_{0}, \ldots, X_{s}$ of $\{0, \ldots, t\}$ such that $\left(c_{j}\right)_{j \in X_{i}}$ is a partition of $b_{i}$ (for each $i \leq s$ ) and a partition $Y_{0}, \ldots, Y_{k}$ of $\{0, \ldots, t\}$ such that $\left(c_{j}\right)_{j \in Y_{i}}$ is a partition of $a_{i}$ (for each $i \leq k)$. Then $\left(t_{n-1}\left(c_{j}\right)\right)_{j \in X_{i}}$ is a partition of $t_{n}\left(b_{i}\right)$ for each $i \leq s$ (by Lemma 7.4(II), and so $\sum_{j \leq t} t_{n-1}\left(c_{j}\right)$ is a decomposition of $\sigma$. Let $\sigma_{i}=\sum_{j \in Y_{i}} t_{n-1}\left(c_{j}\right)$ so that $\sigma_{i} \in \bar{T}_{n-1}\left(a_{i}\right)$ (for each $i \leq k$ ) and $\left(\sigma_{i}\right)_{i \leq k}$ is a partition of $\sigma$.

(2) Suppose $\sum_{j \leq t} \alpha_{j}$ is a decomposition of $\sigma$ and let $X_{0}, \ldots, X_{s}$ be a partition of $\{0, \ldots, t\}$ so that $\left(\alpha_{j}\right)_{j \in X_{i}}$ partitions $t_{n}\left(b_{i}\right)$ (for each $i \leq s$ ). By Lemma 7.4 (2) there exists a partition $\left(c_{j}\right)_{j \leq t}$ of $\mathcal{A}$ into $(n-1)$-indecomposables such that $\left(c_{j}\right)_{j \in X_{i}}$ is a partition of $b_{i}$ and $\left(\alpha_{j}\right)_{j \in X_{i}} \leq_{n-1}\left(t_{n-1}\left(c_{j}\right)\right)_{j \in X_{i}}$ (for each $\left.i \leq s\right)$. Thus, $\left(\alpha_{j}\right)_{j \leq t} \leq_{n-1}\left(t_{n-1}\left(c_{j}\right)\right)_{j \leq t}$.

(3) Suppose $\left(\sigma_{i}\right)_{i \leq k}$ is a partition of $\sigma$ and let $\sum_{j \leq t} \alpha_{j}$ be a decomposition of $\sigma$, where $Y_{0}, \ldots, Y_{k}$ is a partition of $\{0, \ldots, t\}$ with $\sigma_{i}=\sum_{j \in Y_{i}} \alpha_{i}$ (for each $i \leq k$ ). Then, by part (2), there is a partition $\left(c_{j}\right)_{j \leq t}$ of $\mathcal{A}$ into $(n-1)$-indecomposables with $\left(\alpha_{j}\right)_{j \leq t} \leq_{n-1}\left(t_{n-1}\left(c_{j}\right)\right)_{j \leq t}$. Let $\tau_{i}=\sum_{j \in Y_{i}} t_{n-1}\left(c_{j}\right)$ and $a_{i}=\bigvee_{j \in Y_{i}} c_{j}$ so that $\tau_{i} \in \bar{T}_{n-1}\left(a_{i}\right)$ for each $i \leq k$. By Lemma 7.8, we also have $\left(\sigma_{i}\right)_{i \leq k} \leq_{n-1}\left(\tau_{i}\right)_{i \leq k}$.

The following is critical for assigning $n$-back-and-forth invariants to Boolean algebras.

Theorem 7.14. Fix $n \in \omega$. Let $\mathcal{A}$ and $\mathcal{B}$ be Boolean algebras with $\sigma \in \bar{T}_{n}(\mathcal{A})$ and $\tau \in \bar{T}_{n}(\mathcal{B})$. Then

$$
\mathcal{A} \leq_{n} \mathcal{B} \Longleftrightarrow \sigma \leq_{n} \tau .
$$

Proof. The proof is by induction on $n$. The case $n=0$ is trivial. Suppose the theorem holds for $n$. Let $\sigma=\sum_{i \leq k} \alpha_{i} \in \bar{T}_{n+1}(\mathcal{A})$ and $\tau=\sum_{j \leq \ell} \beta_{j} \in \bar{T}_{n+1}(\mathcal{B})$.

$(\Rightarrow)$. Suppose $\mathcal{A} \leq_{n+1} \mathcal{B}$. Let $\left(\tau_{j}\right)_{j \leq t}$ be any partition of $\tau$. By Lemma 7.13(3) there is a partition $\left(b_{j}\right)_{j \leq t}$ of $\mathcal{B}$ and $\rho_{j} \in \bar{T}_{n}\left(b_{j}\right)$ (for each $j \leq t$ ) where $\left(\tau_{j}\right)_{j \leq t} \leq_{n}$ $\left(\rho_{j}\right)_{j \leq t}$. By hypothesis, there is a partition $\left(a_{j}\right)_{j \leq t}$ of $\mathcal{A}$ with $\left(b_{j}\right)_{j \leq t} \leq_{n}\left(a_{j}\right)_{j \leq t}$. By Lemma 7.13 10, there is a partition $\left(\sigma_{j}\right)_{j \leq t}$ of $\sigma$ where $\sigma_{j} \in \bar{T}_{n}\left(a_{j}\right)$ (for each $j \leq t)$. So, by the inductive hypothesis, $\left(\rho_{j}\right)_{j \leq t} \leq_{n}\left(\sigma_{j}\right)_{j \leq t}$. This establishes that $\sigma \leq_{n+1} \tau$.

$(\Leftarrow)$. Suppose $\sigma \leq_{n+1} \tau$. Let $\left(b_{j}\right)_{j \leq s}$ be any partition of $\mathcal{B}$. By Lemma 7.13)(11), there is a partition $\left(\rho_{j}\right)_{j \leq s}$ of $\tau$ with $\rho_{j} \in \bar{T}_{n}\left(b_{j}\right)$ (for each $j \leq s$ ). By hypothesis, there is a partition $\left(\sigma_{j}\right)_{j \leq s}$ of $\sigma$ such that $\left(\rho_{j}\right)_{j \leq s} \leq_{n}\left(\sigma_{j}\right)_{j \leq s}$. By Lemma 7.13(3), there is a partition $\left(a_{j}\right)_{j \leq s}$ and sequence $\left(\gamma_{j}\right)_{j \leq s}$ such that $\gamma_{j} \in \bar{T}_{n}\left(a_{j}\right)$ (for each $j \leq s)$ and $\left(\sigma_{j}\right)_{j \leq s} \leq_{n}\left(\gamma_{j}\right)_{j \leq s}$. Thus $\left(\rho_{j}\right)_{j \leq s} \leq_{n}\left(\gamma_{j}\right)_{j \leq s}$, so by the inductive hypothesis, $\left(b_{j}\right)_{j \leq s} \leq_{n}\left(a_{j}\right)_{j \leq s}$. This establishes that $\mathcal{A} \leq_{n+1} \mathcal{B}$.

It follows from Theorem 7.14 that $\bar{T}_{n}(\mathcal{A}) \subseteq[\sigma]$ for each $\sigma \in \bar{T}_{n}(\mathcal{A})$, where $[\sigma]$ is the class of elements of $\equiv_{n}$-equivalent elements from $\overline{\mathbf{I N V}}_{n}$.

Definition 7.15. For each $n \in \omega$, we define a map $T_{n}$ from Boolean algebras into INV $_{n}$ as follows. Let $\left(a_{i}\right)_{i \leq k}$ be any partition of $\mathcal{A}$ into $n$-indecomposables. Define $T_{n}(\mathcal{A})=\left[\sum_{i \leq k} t_{n}\left(a_{i}\right)\right] \in \mathbf{I N V}_{n}$

It follows from Theorem 7.14 that $T_{n}$ assigns invariants to Boolean algebras for the relation $\leq_{n}$. 
Corollary 7.16. For every $n \in \omega$ and all Boolean algebras $\mathcal{A}$ and $\mathcal{B}$,

$$
\mathcal{A} \leq_{n} \mathcal{B} \Longleftrightarrow T_{n}(\mathcal{A}) \leq_{n} T_{n}(\mathcal{B})
$$

7.5. Computability analysis of $\mathbf{I N V}_{n}$. The goal now is to prove that the relation $\leq_{n}$ on $\overline{\mathbf{I N V}}_{n}$ is computable. It is not necessary to consider all partitions of $\sigma$ and $\tau$ to determine whether $\sigma \leq_{n} \tau$ for $\sigma, \tau \in \overline{\mathbf{I N V}}_{n}$. Instead, it is sufficient to consider only simple partitions of $\sigma$ and $\tau$, which reduces the problem of determining whether $\sigma \leq_{n} \tau$ to check a finite number of conditions.

Definition 7.17. A partition $\left(\sigma_{i}\right)_{i \leq \ell}$ of $\sigma=\sum_{j \leq s} \alpha_{j}$ is a simple partition if there exists a partition $X_{0}, \ldots, X_{\ell}$ of $\{0, \ldots, s\}$ such that for every $i \leq \ell$ either

$$
\sigma_{i}=\sum_{j \in X_{i}}\left(\alpha_{j}\right)_{n-1} \quad \text { or } \quad \sigma_{i}=\gamma_{i}+\sum_{j \in X_{i}}\left(\alpha_{j}\right)_{n-1},
$$

for some $\gamma_{i} \in \bigcup_{j \leq s} \alpha_{j}$ (in the former case $X_{i}$ must be nonempty, while in the latter case $X_{i}$ is allowed to be empty).

Theorem 7.18. Let $\sigma=\sum_{i \leq s} \alpha_{i} \in \overline{\boldsymbol{I N V}}_{n}$ and $\tau=\sum_{j \leq t} \beta_{j} \in \overline{\boldsymbol{I N}}_{n}$. The following are equivalent:

(1) $\sigma \leq_{n} \tau$.

(2) For every decomposition $\sum_{j \leq r} \delta_{j}$ of $\tau$, there exists a simple partition $\left(\sigma_{j}\right)_{j \leq r}$ of $\sigma$ such that $\left(\delta_{j}\right)_{j \leq r} \leq_{n-1}\left(\sigma_{j}\right)_{j \leq r}$.

(3) Both of the following hold:

(a) $\forall \delta \in \bigcup_{j \leq t} \beta_{j} \exists \gamma \in \bigcup_{i \leq s} \alpha_{i}\left(\gamma \geq_{n-1} \delta\right.$ ), (or in other words dc $\bigcup_{j \leq t} \beta_{j}$ $\left.\subseteq \mathrm{dc} \bigcup_{i \leq s} \alpha_{i}\right)$ and

(b) there exists a simple partition $\left(\sigma_{j}\right)_{j \leq t}$ of $\sigma$ such that $\left(\left(\beta_{j}\right)_{n-1}\right)_{j \leq t} \leq_{n-1}$ $\left(\sigma_{j}\right)_{j \leq t}$.

Corollary 7.19. The relation $\leq_{n}$ on $\overline{\mathbf{I N}}_{n}$ is computable. Hence, the structure $\left\langle\boldsymbol{I N} \boldsymbol{V}_{n}, \leq_{n},+, 0,(\cdot)_{n}\right\rangle$ is computably presentable (and, in fact, uniformly in $n$ ).

The proof of the corollary is by induction on $n$ using Theorem 7.18, condition (3).

Proof of Theorem 7.18, (10) $\Longrightarrow$ (2): Consider a decomposition $\sum_{j \leq r} \delta_{j}$ of $\tau$ so that $\left(\delta_{j}\right)_{j \leq r}$ is a partition of $\tau$ into $(n-1)$-bf-types. By (11) there exists a partition $\left(\sigma_{j}\right)_{j \leq r}$ of $\sigma$ such that $\left(\sigma_{j}\right)_{j \leq r} \geq_{n-1}\left(\delta_{j}\right)_{j \leq r}$ for every $j \leq r$, but it might not be simple. Each $\sigma_{j}$ is of the form $\sum_{k \leq m_{j}} \gamma_{j, k}$, where each $\gamma_{j, k}$ either is one of the $(n-1)$-bftypes $\left(\alpha_{i}\right)_{n-1}$ or $\gamma_{j, k} \in{ }^{\mathrm{w}} \alpha_{i}$ for some $i \leq s$. Let $X \subseteq\left\{\langle j, k\rangle: j \leq r, k \leq m_{j}\right\}$ be a set of size $s$ such that for each $i$ there is a unique $\langle j, k\rangle \in S$ with $\gamma_{j, k}=\left(\alpha_{i}\right)_{n-1}$. Then, for each $\langle j, k\rangle \notin X, \gamma_{j, k} \in{ }^{\mathrm{w}} \alpha_{i}$ for some $i \leq s$. Since $\delta_{j} \leq_{n-1} \sum_{k \leq m_{j}} \gamma_{j, k}$ by Lemma 7.12, for each $j \leq r$ there exists $k_{j} \leq m_{j}$ such that $\delta_{j} \leq_{n-1} \gamma_{j, k_{j}}$ and $\delta_{j} \triangleleft_{n-1} \gamma_{j, k}$ for every $k \neq k_{j}$. Let $X_{j}=\left\{k_{j}\right\} \cup\left\{k \leq m_{j}:\langle j, k\rangle \in X\right\}$ and let $\sigma_{j}^{\prime}=\sum_{k \in X_{j}} \gamma_{j, k}$, for each $j$. It follows from Lemma 7.12 that $\delta_{j} \leq_{n-1} \sigma_{j}^{\prime}$. Also note that $\left(\sigma_{j}^{\prime}\right)_{j \leq r}$ is a simple partition of $\sigma$.

(2) $\Longrightarrow$ (1): Follows from Corollary 7.9 .

(2) $\Longrightarrow$ (3): (a) Suppose $\delta \in \bigcup_{j \leq t} \beta_{j}$ and consider the partition of $\tau$ given by $\left(\left(\beta_{0}\right)_{n-1}, \ldots,\left(\beta_{t}\right)_{n-1}, \delta, \ldots, \delta\right)$, where $\delta$ appears $s+2$ times. Let $\left(\sigma_{j}\right)_{j \leq s+t+2}$ be a simple partition of $\sigma$ such that $\sigma_{j} \geq_{n-1}\left(\beta_{j}\right)_{n-1}$ (for $j \leq t$ ) and $\sigma_{j} \geq_{n-1} \delta$ for $t<j \leq s+t+2$. Since the partition is simple, for some $j$ with $t<j \leq t+s+2$, 
we must have $\sigma_{j}=\gamma$ for some $\gamma \in \bigcup_{i \leq k} \alpha_{i}$. It follows that $\forall \delta \in \bigcup_{j \leq t} \beta_{j} \exists \gamma \in$ $\bigcup_{i \leq s} \alpha_{i}\left(\gamma \geq_{n-1} \delta\right)$.

(b) Consider the partition $\left(\left(\beta_{j}\right)_{n-1}\right)_{j \leq t}$ of $\tau$. From (2) we get a simple partition $\left(\sigma_{j}\right)_{j \leq t}$ with $\left(\sigma_{j}\right)_{j \leq t} \geq_{n-1}\left(\left(\beta_{j}\right)_{n-1}\right)_{j \leq t}$.

(3) $\Longrightarrow$ (2): Consider a decomposition $\sum_{j<r} \delta_{j}$ of $\tau$. By rearranging terms, we may also assume that $\delta_{j}=\left(\beta_{j}\right)_{n-1}$ for $j \leq t$ and that for $j>t$ there exists a $k \leq t$ with $\delta_{j} \in{ }^{\mathrm{w}} \beta_{k}$. From condition (b), let $\left(\sigma_{j}\right)_{j \leq t}$ be a simple partition of $\sigma$ such that $\left(\sigma_{j}\right)_{j \leq t} \geq_{n-1}\left(\left(\beta_{j}\right)_{n-1}\right)_{j \leq t}$, and from condition (a), for $j>t$ let $\sigma_{j} \in \bigcup_{i \leq s} \alpha_{i}$ be such that $\sigma_{j} \geq_{n-1} \delta_{j}$. Then $\left(\sigma_{j}\right)_{j \leq r}$ is a simple partition and $\left(\sigma_{j}\right)_{j \leq r} \geq_{n-1}\left(\delta_{j}\right)_{j \leq r}$.

We apply finite sums to provide some counterexamples mentioned in earlier sections.

Example 7.20. There are Boolean algebras $\mathcal{A}$ and $\mathcal{B}$, where $\mathcal{A} \equiv_{5} \mathcal{B}$ and $\mathcal{A}$ is 5 -indecomposable but $\mathcal{B}$ is not. For example, let $\mathcal{A}$ have the 5 -indecomposable type $f_{19}$ and let $\mathcal{B}$ have the 5 -bf-type $f_{5}+f_{17}$. Then $f_{19} \equiv_{5} f_{5}+f_{17}$, but $f_{5}+f_{17}$ is not 5 -indecomposable.

Example 7.21. This example gives a counterexample noted in the comment following Lemma 3.14. There is a Boolean algebra $\mathcal{A}$, a 6 -indecomposable Boolean algebra $\mathcal{B}$ with $\mathcal{A} \leq_{6} \mathcal{B}$, and a partition of $\mathcal{B}$ into 5 -indecomposables with no matching partition of $\mathcal{A}$ into 5 -indecomposables. (It follows by Lemma 3.14 that $\mathcal{A}$ cannot be 6 -indecomposable.) We will use the following 6 -indecomposable types:

- $g_{9}$ has members $\left\{f_{0}, f_{5}\right\}$ and its parent is $f_{5}$.

- $g_{1186}$ has members $\left\{f_{0}, f_{1}, f_{16}, f_{24}, f_{26}\right\}$ and its parent is $f_{17}$.

- $g_{1498}$ has members $\left\{f_{0}, f_{1}, f_{5}, f_{16}, f_{26}\right\}$ and its parent is $f_{19}$.

Let $\mathcal{B}$ be 6 -indecomposable with bf-type $g_{1498}$ and $\mathcal{A}$ have the 6-bf-type $g_{9}+g_{1186}$. Then $\mathcal{A}<_{6} \mathcal{B}$ and the partition of $\mathcal{B}$ into $\left(f_{0}, f_{19}\right)$ can only be matched by a partition of $\mathcal{A}$ into $\left(f_{0}, f_{5}+f_{17}\right)$, but $f_{5}+f_{17}$ is not 5 -indecomposable.

7.6. Finitary isomorphism types. As mentioned is Section 3, a Boolean algebra $\mathcal{A}$ is pseudo-indecomposable if for every $a \in \mathcal{A}$ either $\mathcal{A} \cong \mathcal{A}\lceil a$ or $\mathcal{A} \cong \mathcal{A} \uparrow-a$. A Boolean algebra $\mathcal{A}$ is primitive if every element $a \in \mathcal{A}$ can be partitioned as $a=a_{0} \dot{\vee} \ldots \dot{\vee} a_{n}$, where each subalgebra $\mathcal{A}\left\lceil a_{i}\right.$ is pseudo-indecomposable. Among the pseudo-indecomposable and primitive algebras, the class of finitary Boolean algebras have been singled-out in several very different ways. The name 'finitary' is from the description of these algebras as those in which the set of pseudo-indecomposable isomorphism types of relative subalgebras is finite. However, the description most relevant here is due to Palyutin in Pal71. Let $\mathbf{S}$ be the smallest subset of Boolean algebras which contains the trivial algebra Int(0), the two-element algebra Int(1), and the algebra corresponding to the order-type of the rationals $\operatorname{Int}(\breve{\eta})$, and which is closed under the shuffle-product and free $\omega$-product of finite disjoint sums $\mathcal{A}=\mathcal{A}_{0} \oplus \ldots \oplus \mathcal{A}_{n}$, as described in Section 5 , Then $\mathbf{S}$ is the set of pseudo-indecomposable and finitary Boolean algebras, and the set of all finite disjoint sums of elements of $\mathbf{S}$ is the set of finitary Boolean algebras. (See Hei92 and [Pie89, Section 3.13] for more details.) 
It is not hard to see that each of the algebras constructed in Section [5] $\mathcal{A}_{\alpha}$ for bf-type $\alpha$, is a finitary pseudo-indecomposable Boolean algebra. The converse is also true, that every finitary pseudo-indecomposable Boolean algebra is $n$-indecomposable for every $n$ and has an isomorphic bf-type for sufficiently large $n$. We extend the isomorphism types to $\bigcup_{n} \mathbf{I N V}_{n}$ as the types $\sigma$ for which $T_{n}(\mathcal{A})=\sigma=T_{n}(\mathcal{B}) \Longrightarrow \mathcal{A} \cong \mathcal{B}$. Then the Boolean algebras whose $n$-bf-invariant is an isomorphism type for some $n$ are the finitary Boolean algebras. The details of this are straightforward and laid out in [HMfin.

\section{COMPlEXity ANALYSIS}

In this section we study the complexity of the predicates $\mathrm{R}_{\sigma}$, and prove the quantifier elimination result for $\Sigma_{n}^{c}$ formulas mentioned in the introduction (Theorem 8.12 ).

We begin with a few conventions to simplify our discussion.

Convention 8.1. By Corollary 7.19 the relation $\leq_{n}$ on $\mathbf{I N V}_{n}$ is computable uniformly in $n$, so we will fix representatives $\sigma$ for each of the equivalence classes so that the $n$-indecomposables are among the representatives chosen. We will write $\sigma$ for $[\sigma]$, where $\sigma$ was the chosen representative of this equivalence class. For each $\sigma \in \mathbf{I N V}_{n}$ there is a computable Boolean algebra $\mathcal{A}_{\sigma}$ with $T_{n}\left(\mathcal{A}_{\sigma}\right)=\sigma$. For example, if $\sigma=\sum_{i \leq k} \alpha_{i}$ where $\alpha_{i} \in \mathbf{B F}_{n}$, let $\mathcal{A}_{\alpha_{i}}$ be the computable Boolean algebra produced in Section 5 with $t_{n}\left(\mathcal{A}_{\alpha_{i}}\right)=\alpha_{i}$; then, $\mathcal{A}_{\sigma}=\bigoplus_{i \leq k} \mathcal{A}_{\alpha_{i}}$ satisfies $T_{n}\left(\mathcal{A}_{\sigma}\right)=\sigma$ and is computable. Note that this assignment of Boolean algebras to the elements $\sigma$ is effective.

Convention 8.2. For a Boolean algebra $\mathcal{B}$ and $\sigma \in \mathbf{I N V}_{n}$, we write $\mathcal{B} \leq_{n} \sigma$ when $T_{n}(\mathcal{B}) \leq_{n} \sigma$. Similarly, we write $\sigma \leq_{n} \mathcal{B}$ when $\sigma \leq_{n} T_{n}(\mathcal{B})$.

Definition 8.3. For each $\sigma \in \mathbf{I N V}_{n}$ and Boolean algebra $\mathcal{B}$, the relation $\mathrm{R}_{\sigma}(x)$ is defined by

$$
\left\{b \in \mathcal{B}: \mathcal{B}\left\lceil b \geq_{n} \sigma\right\}\right.
$$

These relations are definable in $L_{\omega_{1} \omega}$ (see [AK00, Lemma 6.6]), but we will show they are also definable in $L_{\omega_{1} \omega}^{c}$ and will study their complexity.

The relations $\mathbf{R}_{\sigma}$ for $\sigma \in \mathbf{I N V}_{n}$ can be expressed using the relations $\mathbf{R}_{\alpha}$ for $\alpha \in \mathbf{B F}_{n}$.

Lemma 8.4. Let $\sigma \in \boldsymbol{I N} \boldsymbol{V}_{n}$ and $\left\langle\left(\alpha_{i, 0}, \ldots, \alpha_{i, m_{i}}\right): i \in \omega\right\rangle$ be a computable listing of tuples from $\mathbf{B F}_{n}$ such that $\sigma \leq_{n} \sum_{j \leq m_{i}} \alpha_{i, j}$ for all $i \in \omega$. Then

$$
\mathrm{R}_{\sigma}(x) \Longleftrightarrow \bigvee_{i \in \omega}\left(\exists y_{i, 0} \dot{\vee} \ldots \dot{\vee} y_{i, m_{i}}=x\right)\left[\bigwedge_{j \leq m_{i}} \mathrm{R}_{\alpha_{i, j}}\left(y_{i, j}\right)\right] .
$$

Proof. Let $\mathcal{B}$ be a Boolean algebra and $x \in \mathcal{B}$. Suppose $\mathcal{B} \models \mathrm{R}_{\sigma}(x)$. Let $y_{0}, \ldots, y_{m}$ be a partition of $x$ into $n$-indecomposables and let $\alpha_{j}=t_{n}\left(y_{j}\right)$. Then, $\sum_{j \leq m} \alpha_{j} \in$ $\bar{T}_{n}\left(\mathcal{B}\lceil x)\right.$ so that $\sum_{j \leq m} \alpha_{j} \equiv_{n} T_{n}(x) \geq_{n} \sigma$. Hence, for some $i$ in the listing $\left\langle\left(\alpha_{i, 0}, \ldots, \alpha_{i, m_{i}}\right): i \in \omega\right\rangle$ we have $m=m_{i}, \alpha_{i, j}=\alpha_{j}$ and $R_{\alpha_{i, j}}\left(y_{j}\right)$ (for each $j \leq m)$. It follows that the right-hand side of the lemma holds. 
Suppose now that for some $i \in \omega, \mathcal{B} \models\left(\exists y_{i, 0} \dot{\vee} \ldots \dot{\vee} y_{i, m_{i}}=x\right)\left[\bigwedge_{j \leq m} \mathrm{R}_{\alpha_{i, j}}\left(y_{i, j}\right)\right]$. Then, by Theorem 2.3 ,

$$
\mathcal{B}\left\lceilx \cong \bigoplus _ { j \leq m _ { i } } \mathcal { B } \left\lceil y_{j} \geq_{n} \sum_{j \leq m_{j}} \alpha_{i, j} \geq_{n} \sigma .\right.\right.
$$

That the list $\left\langle\left(\alpha_{i, 0}, \ldots, \alpha_{i, m_{i}}\right): i \in \omega\right\rangle$ is computable follows from Corollary 7.19,

8.1. Formula satisfaction. The following fact about expressions in the language of Boolean algebras will be used in Definition 8.5 below. For any $\Sigma_{n}$ formula $\varphi\left(x_{0}, \ldots, x_{k}\right)$, there is formula $\psi\left(z_{0}, \ldots, z_{m}\right)$ in $\Sigma_{n}$ such that (i) $\psi$ implies

$$
z_{0} \dot{\vee} \ldots \dot{\vee} z_{m}=1
$$

and (ii) there are subsets $X_{0}, \ldots, X_{k}$ of $\{0, \ldots, m\}$ such that

$$
\exists z_{0}, \ldots, z_{m}\left(\psi\left(z_{0}, \ldots, z_{m}\right) \& x_{0}=\bigvee_{i \in X_{0}} z_{i} \& \ldots \& x_{k}=\bigvee_{i \in X_{k}} z_{i}\right)
$$

is equivalent to $\varphi\left(x_{0}, \ldots, x_{k}\right)$. Furthermore, if $\varphi\left(x_{0}, \ldots, x_{k}\right)$ is in $\Sigma_{n}^{c}$, then $\psi\left(z_{0}, \ldots, z_{m}\right)$ can be found effectively in $\Sigma_{n}^{c}$. Here is the explicit definition of $\psi$ : let $m=2^{k+1}-1$, and let $X_{i}$ be the set of $j \leq m$ whose $i$ th digit in its binary representation is 1 . Then, let $\psi\left(z_{0}, \ldots, z_{m}\right)$ be the formula $z_{0} \dot{V} \ldots \dot{V} z_{m}=$ $1 \& \varphi\left(\bigvee_{i \in X_{0}} z_{i}, \ldots, \bigvee_{i \in X_{k}} z_{i}\right)$

It follows from Corollary 5.4 and Corollary 7.19 that there is a uniformly computable procedure for taking an invariant $\sigma \in \mathbf{I N V}_{n}$ to a Boolean algebra $\mathcal{A}_{\sigma}$, where $T_{n}\left(\mathcal{A}_{\sigma}\right)=\sigma$.

Definition 8.5. For any sentence $\varphi \in \Sigma_{n}$ and invariant $\sigma \in \mathbf{I N V}_{n}$, we write

$$
\sigma \models \varphi
$$

if $\mathcal{A}_{\sigma} \models \varphi$. It follows from Theorem 2.3 and Corollary 7.16 that whenever $T_{n}(\mathcal{B})=$ $\sigma, \mathcal{A}_{\sigma}=\varphi \Longleftrightarrow \mathcal{B} \models \varphi$ for every $\Sigma_{n}$ sentence $\varphi$.

Let $\varphi\left(x_{0}, \ldots, x_{k}\right) \in \Sigma_{n}$, let $\left(\sigma_{0}, \ldots, \sigma_{k}\right)$ be a sequence from $\mathbf{I N V}_{n}$, and let $\sigma=\sigma_{0}+\ldots+\sigma_{k}$. As we noted at the beginning of this subsection, we may assume, without loss of generality, that $\varphi\left(x_{0}, \ldots, x_{k}\right)$ implies $x_{0} \dot{\vee} \ldots \dot{\vee} x_{k}=1$. We define

$$
\left(\sigma_{0}, \ldots, \sigma_{k}\right) \models \varphi\left(x_{0}, \ldots, x_{k}\right)
$$

if in $\mathcal{A}_{\sigma}=\bigoplus_{i=0, \ldots, k} \mathcal{A}_{\sigma_{i}}$ we have $\mathcal{A}_{\sigma}=\varphi\left(e_{0}, \ldots, e_{k}\right)$, where $e_{i} \in \mathcal{A}_{\sigma}$ is

$$
\left(0_{\mathcal{A}_{\sigma_{0}}}, \ldots, 0_{\mathcal{A}_{\sigma_{i-1}}}, 1_{\mathcal{A}_{\sigma_{i}}}, 0_{\mathcal{A}_{\sigma_{i+1}}}, \ldots, 0_{\mathcal{A}_{\sigma_{k}}}\right) .
$$

Lemma 8.6. Let $\varphi\left(x_{0}, \ldots, x_{k}\right)$ be a $\Sigma_{n}^{c}$ formula. Then the set of $\left(\sigma_{0}, \ldots, \sigma_{k}\right)$ such that

$$
\left(\sigma_{0}, \ldots, \sigma_{k}\right) \models \varphi\left(x_{0}, \ldots, x_{k}\right)
$$

is $\Sigma_{n}^{0}$.

Proof. For any computable Boolean algebra $\mathcal{A}$ and partition $\left(a_{i}\right)_{i \leq k}$, the question of whether $\mathcal{A} \models \varphi\left(a_{0}, \ldots, a_{k}\right)$ is $\Sigma_{n}^{0}$ by [AK00, Theorem 8.6]. 
$\Sigma_{n}^{c}$ formulas can be put into a special form:

Theorem 8.7. For every $\Sigma_{n+1}^{c}$ formula $\varphi(x)$, there are $0^{(n)}$-computable enumerations of tuples $\left\langle\left(\alpha_{i, 0}, \ldots, \alpha_{i, m_{i}}\right): i \in \omega\right\rangle$ and $\left\langle\left(\beta_{i, 0}, \ldots, \beta_{i, m_{i}}\right): i \in \omega\right\rangle$ from $\mathbf{B F}_{n}$ such that $\varphi(x)$ is equivalent to

$$
\begin{aligned}
\bigvee_{i \in \omega}\left(\exists y_{i, 0} \dot{\vee} \ldots \dot{\vee} y_{i, m_{i}}=x\right)\left(\exists z_{i, 0} \dot{\vee} \ldots \dot{\vee} z_{i, m_{i}}=-x\right) & \\
& \times\left[\bigwedge_{j=0}^{m_{i}} \mathrm{R}_{\alpha_{i, j}}\left(y_{i, j}\right) \& \bigwedge_{j=0}^{m_{i}} \mathrm{R}_{\beta_{i, j}}\left(z_{i, j}\right)\right] .
\end{aligned}
$$

Proof. The existence of the $0^{(n)}$-computable enumeration of tuples will be shown uniformly in $\varphi$, so it is sufficient to show the theorem for the special case when $\varphi$ is of the form $\exists x_{0}, \ldots, x_{k} \psi\left(x, x_{0}, \ldots, x_{k}\right)$, with $\psi$ being $\Pi_{n}^{c}$. Then, we dovetail the computations for arbitrary computable disjunctions. Furthermore, we can assume $\varphi$ is of the form

$$
\left(\exists y_{0} \dot{\vee} \ldots \dot{\vee} y_{k}=x\right)\left(\exists z_{0} \dot{\vee} \ldots \dot{\vee} z_{k}=-x\right) \psi\left(y_{0}, \ldots, y_{k}, z_{0}, \ldots, z_{k}\right)
$$

by setting $y_{j}=x_{j} \wedge x$ and $z_{j}=x_{j} \wedge-x$.

The set of tuples from $\mathbf{I N V}_{n}$ such that

$$
\left(\sigma_{0}, \ldots, \sigma_{k}, \tau_{0}, \ldots, \tau_{k}\right) \models \psi\left(y_{0}, \ldots, y_{k}, z_{0}, \ldots, z_{k}\right)
$$

is $\Pi_{n}^{0}$ and so is computable from $0^{(n)}$. Let $\left\langle\left(\sigma_{i, 0}, \ldots, \sigma_{i, k}, \tau_{i, 0}, \ldots, \tau_{i, k}\right): i \in \omega\right\rangle$ list these tuples. We will first show that for any Boolean algebra $\mathcal{A}, \mathcal{A}=\varphi$ if and only if

$$
\begin{aligned}
\mathcal{A}=\bigvee_{i \in \omega}\left(\exists y_{0} \dot{\vee} \ldots \dot{\vee} y_{k}=x\right)\left(\exists z_{0} \dot{\vee} \ldots \dot{\vee} z_{k}=-x\right) \\
\times\left[\bigwedge_{j \leq k} \mathrm{R}_{\sigma_{i, j}}\left(y_{j}\right) \& \bigwedge_{j \leq k} \mathrm{R}_{\tau_{i, j}}\left(z_{j}\right)\right] .
\end{aligned}
$$

Suppose first that $\mathcal{A} \models \varphi$. Thus, there exists a partition $\left(a_{0}, \ldots, a_{k}\right)$ of $x$ and a partition $\left(b_{0}, \ldots, b_{k}\right)$ of $-x$ such that $\mathcal{A} \models \psi\left(a_{0}, \ldots, a_{k}, b_{0}, \ldots, b_{k}\right)$. Let $T_{n}\left(a_{j}\right)=\sigma_{i, j}$ and $T_{n}\left(b_{j}\right)=\tau_{i, j}$, where $\left(\sigma_{i, 0}, \ldots, \sigma_{i, k}, \tau_{i, 0} \ldots, \tau_{i, k}\right)$ is in the enumeration. Therefore, (2) holds.

Suppose now that (2) holds as witnessed by $i$ and elements $a_{0}, \ldots, a_{k}, b_{0}, \ldots, b_{k}$. Then $T_{n}\left(a_{j}\right) \geq_{n} \sigma_{i, j}$ and $T_{n}\left(b_{j}\right) \geq_{n} \tau_{i, j}$ for each $j \leq k$. It follows from Theorem 2.3 that since $\varphi$ is $\Pi_{n}^{0}$ and $\left(\sigma_{i, 0}, \ldots, \sigma_{i, k}, \tau_{i, 0} \ldots, \tau_{i, k}\right) \models \psi\left(y_{0}, \ldots, y_{k} z_{0}, \ldots, z_{k}\right)$, we have that $\mathcal{A} \models \psi\left(a_{0}, \ldots, a_{k}, b_{0}, \ldots, b_{k}\right)$.

By Lemma 8.4 each $\mathrm{R}_{\sigma}$ is equivalent to a formula of the form

$$
\mathrm{R}_{\sigma}(y) \Longleftrightarrow \bigvee_{i \in \omega}\left(\exists y_{i, 0} \dot{\vee} \ldots \dot{\vee} y_{i, m_{i}}=y\right)\left[\bigwedge_{j \leq m} \mathrm{R}_{\alpha_{i, j}}\left(y_{i, j}\right)\right]
$$

for some computable sequence $\left\langle\left(\alpha_{i, 0}, \ldots, \alpha_{i, m_{i}}\right): i \in \omega\right\rangle$ from $\mathbf{B F}_{n}$. Use this equivalence to replace each occurrence of $\mathrm{R}_{\sigma_{i, j}}$ and $\mathrm{R}_{\tau_{i, j}}$ in (2); then, rewrite the formula as a disjunction of existential formulas. Note that we obtain a formula of the form we wanted for the theorem. 
The theorem above can be easily extended to formulas with more than one variable $\varphi\left(x_{0}, \ldots, x_{k}\right)$ as in the corollaries below. When restricted to sentences, the theorem is as follows:

Corollary 8.8. For every $\Sigma_{n+1}^{c}$ sentence $\varphi$, there is a $0^{(n)}$-computable enumeration of tuples $\left\langle\left(\alpha_{i, 0}, \ldots, \alpha_{i, m_{i}}\right): i \in \omega\right\rangle$ from $\mathbf{B F}_{n}$ such that

$$
\varphi \Longleftrightarrow \bigvee_{i \in \omega}\left(\exists y_{i, 0} \dot{\vee} \ldots \dot{\vee} y_{i, m_{i}}=1\right)\left[\bigwedge_{j=0}^{m_{i}} \mathrm{R}_{\alpha_{i, j}}\left(y_{i, j}\right)\right] .
$$

Proof. The proof is essentially the same proof as the one of the theorem above but without considering $x$.

Corollary 8.9. For every $\Sigma_{n+1}^{c}$ formula $\varphi\left(x_{0}, \ldots, x_{k}\right)$ which implies $x_{0} \dot{\vee} \ldots \dot{\vee} x_{k}$ $=1$, there are $0^{(n)}$-computable enumerations of tuples $\left\langle\left(\alpha_{\ell, i, 0}, \ldots, \alpha_{\ell, i, m_{\ell, i}}\right): i \in \omega\right\rangle$ from $\mathbf{B F}_{n}$, one for each $\ell \leq k$, such that

$$
\begin{aligned}
\varphi\left(x_{0}, \ldots, x_{k}\right) \Longleftrightarrow & x_{0} \dot{\vee} \ldots \dot{\vee} x_{k}=1 \\
& \& \bigwedge_{\ell=0}^{k} \bigvee_{i \in \omega}\left(\exists y_{i, 0} \dot{\vee} \ldots \dot{\vee} y_{i, m_{\ell, i}}=x_{\ell}\right)\left[\bigwedge_{j=0}^{m_{\ell, i}} \mathrm{R}_{\alpha_{\ell, i, j}}\left(y_{i, j}\right)\right] .
\end{aligned}
$$

Proof. The proof is essentially the same proof as the one of the theorem above but using $x_{0}, \ldots, x_{k}$ instead of $x$ and $-x$.

Let $\mathcal{B}$ be a Boolean algebra and $\sigma \in \mathbf{I N V}_{n}$. We now turn to the study of the complexity of the following relations on $\mathcal{B}$ :

$$
\left\{x \in \mathcal { B } : \mathcal { B } \lceil x \geq _ { n } \sigma \} \quad \text { and } \quad \left\{x \in \mathcal{B}: \mathcal{B}\left\lceil x \leq_{n} \sigma\right\} .\right.\right.
$$

Lemma 8.10. Let $\sigma \in \boldsymbol{I N} \boldsymbol{V}_{n}$. Then there is a $\Pi_{n}^{c}$ formula $\varphi_{\sigma}$ such that for any Boolean algebra $\mathcal{B}$,

$$
\mathcal{B}\left\lceil x \geq_{n} \sigma \quad \Longleftrightarrow \quad \mathcal{B} \models \varphi_{\sigma}(x)\right.
$$

In particular, the relations $\mathrm{R}_{\alpha}(x)$ are $\Pi_{n}^{c}$-definable for each $\alpha \in \mathbf{B F}_{n}$.

Proof. The proof is by induction on $n$, where the case of $n=0$ is trivial. Therefore, assume the predicates $\mathrm{R}_{\tau}(x)$ are $\Pi_{n-1}^{c}$ whenever $\tau \in \mathbf{I N} \mathbf{V}_{n-1}$.

By Theorem 7.18, $\mathcal{B}\left\lceil b \geq_{n} \sigma\right.$ if and only if for every partition $b=b_{0} \dot{V} \ldots \dot{\vee} b_{k}$ with $T_{n-1}\left(b_{i}\right)=\beta_{i}$, there exists a simple partition $\left(\rho_{i}\right)_{i \leq k}$ of $\sigma$ such that $\beta_{i} \leq_{n-1}$ $\rho_{i}$ for every $i$. Equivalently, $\mathcal{B}\left\lceil b \geq_{n} \sigma\right.$ if and only if for every partition $b=$ $b_{0} \dot{\vee} \ldots \dot{\vee} b_{k}$ and for every sequence $\left(\beta_{i}\right)_{i \leq k} \subseteq \mathbf{B F}_{n-1}$ such that $\mathcal{B}\left\lceil b_{i} \geq_{n-1} \beta_{i}\right.$, there exists a simple partition $\left(\rho_{i}\right)_{i \leq k}$ of $\sigma$ such that for every $i, \beta_{i} \leq_{n-1} \rho_{i}$. (This equivalence uses the ideas in the proof of Theorem 7.18)

Let $I_{\sigma}$ be the set of sequences $\left(\beta_{i}\right)_{i \leq k} \subseteq \mathbf{B F}_{n-1}$ for which there is no simple partition $\left(\rho_{i}\right)_{i \leq k}$ of $\sigma$ with $\left(\beta_{i}\right)_{i \leq k} \leq_{n-1}\left(\rho_{i}\right)_{i \leq k}$. The set $I_{\sigma}$ is computable by Corollary 7.19 and the fact that there are only finitely many simple partitions of $\sigma$ of length $k$. The sentence $\varphi_{\sigma}$ will say that for any $\left(\beta_{i}\right)_{i \leq k} \in I_{\sigma}$ there is no partition $\left(b_{i}\right)_{i \leq k}$ of $b$ with $\mathcal{B}\left\lceil b_{i} \geq_{n-1} \beta_{i}\right.$ for each $i \leq k$; that is,

$$
\varphi_{\sigma}(x) \equiv \bigwedge_{\left(\beta_{i}\right)_{i \leq k} \in I_{\sigma}} \neg\left(\exists x_{0} \dot{\vee} \ldots \dot{\vee} x_{k}=x\right)\left[\bigwedge_{i \leq k} \mathrm{R}_{\beta_{i}}\left(x_{i}\right)\right] .
$$

$\varphi_{\sigma}$ is $\Pi_{n}^{c}$ from the inductive hypothesis and is equivalent to $\mathrm{R}_{\sigma}(x)$. 
Lemma 8.11. Let $\sigma \in \boldsymbol{I N} \boldsymbol{V}_{n}$. Then there exists a $\Pi_{n+1}^{c}$ formula $\psi_{\sigma}$ such that for any Boolean algebra $\mathcal{B}$,

$$
\mathcal{B}\left\lceil x \leq_{n} \sigma \quad \Longleftrightarrow \quad \mathcal{B} \models \psi_{\sigma}(x) .\right.
$$

Proof. Let $J_{\sigma}$ be the set of $\tau \in \mathbf{I N V}_{n}$ with $\tau \not_{n} \sigma$. This set is computable by Corollary 7.19, Then, $\mathcal{B}\left\lceil b \leq_{n} \sigma\right.$ if and only if for no $\tau \in J_{\sigma}$ is it the case that $\tau \leq_{n} \mathcal{B}\left\lceil b\right.$. Let $\psi_{\sigma}$ express this; that is,

$$
\bigwedge_{\tau \in J_{\sigma}} \neg \mathrm{R}_{\tau}(x) .
$$

By Lemma $8.10 \psi_{\sigma}$ is $\Pi_{n+1}^{c}$.

Theorem 8.12. Let $\mathcal{B}$ be a Boolean algebra, $R \subseteq \mathcal{B}$ and $n \in \omega$. The following are equivalent:

(1) $R$ is relatively intrinsically $\Sigma_{n+1}$. That is, if $\mathcal{A} \cong \mathcal{B}$ and $(\mathcal{A}, Q) \cong(\mathcal{B}, R)$, then $Q$ is a $\Sigma_{n+1}^{0}(\mathcal{A})$ subset of $\mathcal{A}$.

(2) $R$ is explicitly $\Sigma_{n+1}$. That is, $R$ can be defined in $\mathcal{B}$ by a computable infinitary $\Sigma_{n+1}^{c}$ formula.

(3) There is a $0^{(n)}$-computable sequence $\left\{\varphi_{i}: i \in \omega\right\}$ of finitary $\Sigma_{1}$ formulas that use the predicates $\mathbf{R}_{\alpha}$, for $\alpha \in \mathbf{B F}_{n}$, such that

$$
x \in R \Longleftrightarrow \bigvee_{i \in \omega} \varphi_{i}(x) \text {. }
$$

Proof. For (1) $\Leftrightarrow(2)$ see AK00, Theorem 10.1].

$(2) \Rightarrow(3)$. This is essentially Theorem 8.7

$(3) \Rightarrow(2)$. By Lemma 8.10 , each $\mathrm{R}_{\alpha}$ is given by a $\Pi_{n}^{c}$ formula uniformly in $\alpha \in \mathbf{B F}_{n}$. So, from the hypothesis, there is a $0^{(n)}$-computable set of $\Sigma_{n+1}^{c}$ formulas, $\left\{\psi_{i}: i \in \omega\right\}$, such that

$$
x \in R \Longleftrightarrow \bigvee_{i \in \omega} \psi_{i}(x)
$$

It follows from [AK00, Proposition 7.14] that there is a $\Sigma_{n+1}^{c}$ formula $\chi(x)$ equivalent to $\bigvee_{i \in \omega} \psi_{i}(x)$.

Recall that a presentation of a Boolean algebra $\mathcal{B}$ is $n$-approximable if its $\Sigma_{n+1^{-}}^{c}$ diagram is $\Sigma_{n+1}^{0}$; in other words, if the set of $\Sigma_{n+1}^{c}$-formulas with parameters true in $\mathcal{B}$ is a set c.e. in $0^{(n)}$.

Theorem 8.13. Let $\mathcal{B}$ be a presentation of a Boolean algebra. The following are equivalent:

(1) The $\Sigma_{n+1}^{c}$-diagram of $\mathcal{B}$ is $\Sigma_{n+1}^{0}$.

(2) The relations $\mathrm{R}_{\alpha}(\mathcal{B})$ are computable in $0^{(n)}$ for each $\alpha \in \mathbf{B F}_{n}$.

Proof. (11) $\Longrightarrow$ (2): The relations $\left\{x \in \mathcal{B}: \mathcal{B}\left\lceil x \geq_{n} \alpha\right\}\right.$ are $\Pi_{n}^{c}$ definable in $\mathcal{B}$. Therefore, in particular, they are $\Sigma_{n+1}^{c}$ and $\Pi_{n+1}^{c}$. Then, by (11), these relations are $\Delta_{n+1}^{0}$ as wanted.

(2) $\Longrightarrow$ (11): By Corollary 8.8. every $\Sigma_{n+1}^{c}$-sentence $\varphi$ is equivalent to one of the form

$$
\bigvee_{i \in \omega}\left(\exists x_{0} \dot{\vee} \ldots \dot{\vee} x_{m_{i}}=1\right)\left[\bigwedge_{j=0}^{m_{i}} \mathrm{R}_{\alpha_{i, j}}\left(x_{j}\right)\right],
$$


where $\alpha_{i, j} \in \mathbf{B F}_{n}$ and the sequence of formulas in the disjunction is computable in $0^{(n)}$ uniformly in $\varphi$. Therefore, if $\mathcal{B} \models \varphi, 0^{(n)}$ will eventually find a witnesses $i$ and a partition $a_{0} \dot{\vee} \ldots \dot{\vee} a_{m_{i}}=1$ such that $\bigwedge_{j=0}^{m_{i}} \mathrm{R}_{\alpha_{i, j}}\left(a_{j}\right)$ and thus whether $\mathcal{B} \models \varphi$ is $\Sigma_{n+1}^{0}$. To decide the $\Sigma_{n+1}^{c}$-formulas $\varphi(b)$ with $b \in \mathcal{B}$, we need to use Theorem 8.7. For formulas with more variables we need Corollary 8.9 .

\section{ACKNOWLEDGMENTS}

The authors would like to thank Pavel Alaev for comments on a previous draft and the anonymous referee for a detailed report. They would also like to thank Mushfeq Khan and Ashutosh Kumar for finding a mistake in an earlier version of Theorem 5.3 .

\section{REFERENCES}

[AK90] C. J. Ash and J. F. Knight. Pairs of recursive structures. Ann. Pure Appl. Logic, 46(3):211-234, 1990. MR1049387 (91b:03061)

[AK00] C. J. Ash and J. Knight. Computable Structures and the Hyperarithmetical Hierarchy. Elsevier Science, 2000. MR1767842 (2001k:03090)

[Ala04] Pavel Alaev. Computable homogeneous Boolean algebras and a Metatheorem. Algebra and Logic, 43(2):133-158, 2004. MR2072567 (2005d:03082)

[Ash86a] C. J. Ash. Recursive labelling systems and stability of recursive structures in hyperarithmetical degrees. Trans. Amer. Math. Soc., 298(2):497-514, 1986. MR860377 (87j:03060)

[Ash86b] C. J. Ash. Stability of recursive structures in arithmetical degrees. Ann. Pure Appl. Logic, 32(2):113-135, 1986. MR863330 (88j:03021)

[Ash87] C. J. Ash. Categoricity in hyperarithmetical degrees. Ann. Pure Appl. Logic, 34(1):114, 1987. MR887551 (88e:03053)

[Bar73] J. Barwise. Back and forth through infinitary logic. In M. D. Morley, editor, Studies in model theory, pages 5-34. The Mathematical Association of America, Buffalo, N.Y., 1973. MR0342370 (49:7116)

[Bar95] Ewan J. Barker. Back and forth relations for reduced abelian p-groups. Ann. Pure Appl. Logic, 75(3):223-249, 1995. MR1355134 (96j:03066)

[Cal05] Wesley Calvert. The isomorphism problem for computable abelian $p$-groups of bounded length. J. Symbolic Logic, 70(1):331-345, 2005. MR2119136 (2005j:03033)

[CMS06] Barbara F. Csima, Antonio Montalbán, and Richard A. Shore. Boolean algebras, Tarski invariants, and index sets. Notre Dame Journal of Formal Logic, 47(1):1-23, 2006. MR2211179 (2006k:03081)

[Dic85] M.A. Dickman. Larger infinitary languages. In J. Barwise and S. Feferman, editors, Larger Infinitary Languages, chapter Chapter IX, pages pp. 316-353. Springer, 1985. MR819540

[DJ94] Rod Downey and Carl G. Jockusch. Every low Boolean algebra is isomorphic to a recursive one. Proc. Amer. Math. Soc., 122(3):871-880, 1994. MR1203984(95a:03044)

[FZ80] Jörg Flum and Martin Ziegler. Topological model theory, volume 769 of Lecture Notes in Mathematics. Springer, Berlin, 1980. MR560706 (81j:03059)

[Hei81] Lutz Heindorf. Comparing the expressive power of some languages for Boolean algebras. Z. Math. Logik Grundlag. Math., 27(5):419-434, 1981. MR628302 (82m:03050)

[Hei92] Lutz Heindorf. Alternative characterizations of finitary and well-founded Boolean algebras. Algebra Universalis, 29: 109-135, 1992. MR.1145559(93b:06027)

[HMfin] Kenneth Harris and Antonio Montalbán. Boolean algebras of finitary type and the back-and-forth hierarchy.

[JS94] Carl G. Jockusch and Robert I. Soare. Boolean algebras, Stone spaces, and iterated Turing jumps. J. Symbolic Logic, 59(4):1121-1138, 1994. MR1312300 (95m:03094)

[KS00] Julia F. Knight and Michael Stob. Computable Boolean algebras. J. Symbolic Logic, 65(4):1605-1623, 2000. MR 1812171 (2001m:03086)

[Mon89] J.D. Monk, editor. Handbook of Boolean algebras, Vol. 1. North-Holland, 1989. MR991565 (90k:06002) 
[Pal71] E.A. Palyutin. Boolean algebras having a categorical weak second-order theory (in Russian). Algebra i Logika, 10:523-534, 1971. MR0304167 (46:3302)

[Pie89] R. S. Pierce. Countable Boolean algebras. In Handbook of Boolean algebras, Vol. 3, pages 775-876. North-Holland, Amsterdam, 1989. MR991610

[Thu95] John J. Thurber. Every low 2 Boolean algebra has a recursive copy. Proc. Amer. Math. Soc., 123(12):3859-3866, 1995. MR:1283564(96b:03047)

E-mail address: kenneth@kaharris.org

$U R L$ : http://kaharris.org

Department of Mathematics, University of Chicago, Chicago, Illinois 60637-1538

E-mail address: antonio@math.uchicago.edu

$U R L$ : www.math.uchicago.edu/ antonio 